University of Louisville

ThinkIR: The University of Louisville's Institutional Repository

$5-2020$

\title{
Joint location and inventory models and algorithms for deployment of hybrid electric vehicle charging stations.
}

Jie Zhang

University of Louisville

Follow this and additional works at: https://ir.library.louisville.edu/etd

Part of the Operational Research Commons

\section{Recommended Citation}

Zhang, Jie, "Joint location and inventory models and algorithms for deployment of hybrid electric vehicle charging stations." (2020). Electronic Theses and Dissertations. Paper 3378.

https://doi.org/10.18297/etd/3378

This Doctoral Dissertation is brought to you for free and open access by ThinkIR: The University of Louisville's Institutional Repository. It has been accepted for inclusion in Electronic Theses and Dissertations by an authorized administrator of ThinkIR: The University of Louisville's Institutional Repository. This title appears here courtesy of the author, who has retained all other copyrights. For more information, please contact thinkir@louisville.edu. 


\title{
JOINT LOCATION AND INVENTORY MODELS AND ALGORITHMS FOR DEPLOYMENT OF HYBRID ELECTRIC VEHICLE CHARGING STATIONS
}

\author{
By \\ Jie Zhang \\ M.S., University At Buffalo, 2012
}

\begin{abstract}
A Dissertation
Submitted to the Faculty of the

J.B. Speed School of Engineering of the University of Louisville in Partial Fulfillment of the Requirements

for the Degree of

Doctor of Philosophy

in Industrial Engineering
\end{abstract}

Department of Industrial Engineering

University of Louisville

Louisville, Kentucky

May 2020 



\title{
JOINT LOCATION AND INVENTORY MODELS AND ALGORITHMS FOR DEPLOYMENT OF HYBRID ELECTRIC VEHICLE CHARGING STATIONS
}

\author{
By \\ Jie Zhang \\ M.S., University At Buffalo, 2012
}

A Dissertation Approved On

April 22, 2020

by the following Dissertation Committee

Dr. Lihui Bai, Dissertation Director

Dr. Suraj M. Alexander

Dr. Erin L. Gerber

Dr. Dar-Jen Chang 


\section{ACKNOWLEDGMENTS}

I am highly indebted to my dissertation advisor, Dr. Lihui Bai, for her constant support and supervision. With almost maternal solicitude she urged me to make me naturally open to her unreserved knowledge and experience. Her attitude of devoting inspires me all the way of my research life. My sincere thanks go to Dr. Tongdan Jin, for giving me the great opportunity to participant in this interesting topic and endless help for my research work. I also want to thank Dr. Alexander, Dr. Bae, Dr. Gerber, And Dr. Chang for reviewing and giving comments to make my dissertation better.

My deep appreciation goes to my beloved parents, Jin Zhao and Dr. Xinkai Zhang for their love and support. Their encouragement is always my strong backup and makes me confident again. Besides, I feel grateful to my cousion Dr. Yi Tan and cousin-in-law Ling-Bing Kung for giving me a warn home in the U.S..

Last but not least, I would like to thank my fellow friends for their support and encouragement. All the works and joys we shared have been priceless gifts for my future life. 


\begin{abstract}
JOINT LOCATION AND INVENTORY MODELS AND ALGORITHMS FOR DEPLOYMENT OF HYBRID ELECTRIC VEHICLE CHARGING STATIONS
\end{abstract}

Jie Zhang

April 24, 2020

This thesis describes a study of a novel concept of hybrid electric vehicle charging stations in which two types of services are offered: battery swapping and fast level-3 DC charging. The battery swapping and fast-charging service are modeled by using the $\mathrm{M} / \mathrm{G} / \mathrm{s} / \mathrm{s}$ model and the $\mathrm{M} / \mathrm{G} / \mathrm{s} / \infty$ model, respectively. In particular, we focus on the operations of joint battery swapping and fast charging services, develop four joint locations and inventory models: two for the deployment of battery swapping service, two for the deployment of hybrid electric vehicle charging service. The first model for each deployment system considers a service-level constraint for battery swapping and hybrid charging service, whereas the second for each deployment system considers total sojourn time in stations. The objective of all four models is to minimize total facility setup cost plus battery and supercharger purchasing cost. The service level, which is calculated by the Erlang loss function, depends on the stockout probability for batteries with enough state of charge (SOC) for the battery swapping service and the risk of running out of superchargers for the quick charging service. The total sojourn time is defined as the sum of the service time and the waiting time in the station. Metaheuristic algorithms using a Tabu search are developed to tackle the proposed nonlinear mixed-integer optimization model. Computational results on randomly generated 
instances and on a real-world case comprised of 714,000 households show the efficacy of proposed models and algorithms. 


\section{TABLE OF CONTENTS}

Page

ACKNOWLEDGMENTS

ABSTRACT

LIST OF TABLES $\quad$ ix

LIST OF FIGURES $\quad$ xi

CHAPTER

1 INTRODUCTION 1

2 LITERATURE REVIEW 5

2.1 Estimate of EV Charging Demand . . . . . . . . . . . 5

2.2 Design of Electric Vehicle battery swapping Station . . . . . . 6

2.3 Facility Location . . . . . . . . . . . . . . . . 9

2.4 Inventory Control with Service Level . . . . . . . . . . . . . . . 12

2.5 Joint Inventory-location Model . . . . . . . . . . . . . . . . . . 14

2.6 Summary of Literature . . . . . . . . . . . . . . . . 15

3 JOINT FACILITY LOCATION AND INVENTORY MODELS FOR BATTERY SWAPPING STATION SERVICES 16

3.1 Introduction . . . . . . . . . . . . . . . . 16

3.2 Research Contribution . . . . . . . . . . . . . . . 18

3.3 Notation . . . . . . . . . . . . . . . . . 19

3.4 Modeling EV Arrival and Battery Charging Service Using M/G/s/s 20

3.4.1 Model and data analysis . . . . . . . . . . . . . 20

3.4 .2 The Erlang loss model . . . . . . . . . . . . . 21

3.4.3 The Erlang queueing model . . . . . . . . . . . . . 24 
3.5 The Mathematical Models . . . . . . . . . . . . . .

3.5.1 Optimal location and inventory for BSS with service-level

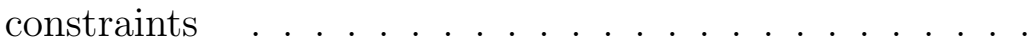

3.5.2 Optimal location and inventory model for battery swapping station with service-time constraint . . . . . . . 27

3.6 Solution Approaches . . . . . . . . . . . . . . . . . . . . 28

3.6.1 Relationship between $s_{j}$ and $\theta_{j} \ldots \ldots \ldots \ldots \ldots$

3.6.2 A metaheuristic algorithm for the OLIBSS-SL and the OLIBSS-ST models . . . . . . . . . . . . . . . . . 32

3.7 An Illustrative Example for the Metaheuristic Method . . . . . 38

4 JOINT FACILITY LOCATION AND INVENTORY MODELS FOR NOVEL HYBRID CHARGING STATION 40

4.1 Introduction . . . . . . . . . . . . . . . . . 40

4.2 Research Contribution . . . . . . . . . . . . . . . . . . . . 41

4.3 Notation . . . . . . . . . . . . . . . . . . . . . . . . . 42

4.4 Modeling EV Supercharging Service Using M/G/s/ $\infty \ldots .44$

4.4 .1 Model and data analysis . . . . . . . . . . . . . . . . 44

4.4 .2 Electric power demand . . . . . . . . . . . . . 45

4.5 The Mathematical Model . . . . . . . . . . . . . . . . . . 46

4.5.1 Optimal location and inventory for hybrid charging station with service-level constraints _ . . . . . . . . . . 46

4.5.2 Optimal location and inventory for hybrid charging station with service time constraints f . . . . . . . . . 48

4.6 Solution Approaches . . . . . . . . . . . . . . . . . 50

4.6.1 The metaheuristic solution method . . . . . . . . . 50

4.6.2 A metaheuristic algorithm for the OLIHCS-SL and the OLIHCS-ST models . . . . . . . . . . . . . . . . . 51

4.7 An illustrative example for the metaheuristic method _ . . . 56 
5 COMPUTATIONAL RESULTS 58

5.1 EV Charging-Demand Forecasting . . . . . . . . . 58

5.2 Settings of Other Parameters in the Four Models . . . . . . . 62

5.3 Numerical Results for the OLIBSS-SL Model . . . . . . . . . . 63

5.4 Numerical Results for the OLIBSS-ST Model . . . . . . . . . 66

5.5 Numerical Results for the OLIHCS-SL Model . . . . . . . . 70

5.6 Numerical Results for the OLIHCS-ST Model . . . . . . . . . 72

5.7 Case Study for Mid-Ohio Region _ . . . . . . . . . . . 76

6 CONCLUSION AND FUTURE RESEARCH 82

REFERENCES $\quad 84$

$\begin{array}{ll}\text { CURRICULUM VITAE } & 94\end{array}$ 


\section{LIST OF TABLES}

TABLE Page

1 Sets and indices. . . . . . . . . . . . . . . . . . . . . . . 19

2 Parameters and indices. . . . . . . . . . . . . . 19

3 Decision variables. . . . . . . . . . . . . . . . . . 19

4 Results of linear regression test for Erlang loss function. . . . . . . . . 29

5 Results of linear regression test for Erlang queueing function. . . . . 30

6 Data for TAZs and candidate locations. . . . . . . . . . . . 38

$7 \quad$ Sets and indices. . . . . . . . . . . . . . . . . 42

8 Parameters and indices. . . . . . . . . . . . . . . . . 42

9 Decision variables. . . . . . . . . . . . . . . . . . 43

10 Data for TAZs and candidate-station locations. . . . . . . . . . 56

11 Sets and indices . . . . . . . . . . . . . . . . 60

12 Parameters and indices. . . . . . . . . . . . . . . 60

13 Decision variables. . . . . . . . . . . . . . . . 60

14 An example distance table. . . . . . . . . . . . . . . . . . 61

15 The result of EV charging-demand forecasting example. . . . . . . . . 61

16 Charging topologies. . . . . . . . . . . . . . . 62

17 Network size of five experimental sets. . . . . . . . . . . . . 63

18 Average results of the OLIBSS-SL model with $\gamma=0.2 . \quad \ldots . . . .64$

19 Total cost $(\times \$ 1,000)$ and number of stations with various $\gamma . \quad \ldots$. . 64

20 Average results for the OLIBSS-ST model for $W_{\max }=10$ minutes. $\quad$. $\quad 66$

21 Total cost $(\times \$ 1,000)$ and number of stations for various $W_{\max }$. . . 67

22 Averaged results for the OLIHCS-SL model $(\gamma=0.2, \beta=0.2) \ldots$. . 70

23 Sensitivity analysis of cost of the OLIHCS-SL model $(\times 1000)$ and number of stations for various $\gamma$ and $\beta$. . . . . . . . . . . . . 71 
24 Averaged results for the OLIHCS-ST model with $W_{\max }=10$ minutes. 72

25 Total cost $(\times \$ 1,000)$ and number of stations for various $W_{\max }$. . . 73

26 Average number of batteries for various $W_{\max } . \quad \ldots \ldots$. . . . . . 74

27 Average number of superchargers for various $W_{\max }$. . . . . . . . . 75

28 Sample data for case study (note: $i, k$, and $l$ are TAZ indices). . . . 76

29 Results for the OLIHCS-SL model for various charging rates. . . . . 80

30 Results for the OLIHCS-ST model for various charging rates. . . . . 81 


\section{LIST OF FIGURES}

FIGURE $\quad$ Page

1 Battery swapping station with priority queue. . . . . . . . 16

2 The $\mathrm{M} / \mathrm{G} / \mathrm{s} / \mathrm{s}$ queueing model. . . . . . . . . . . . . . . . . . . 21

3 Inventory required for different arrival rates for target $\gamma \ldots \ldots$

4 Inventory required for different arrival rates for target $W_{\max }$. . . . 31

5 The flow chart of the metaheuristic for the OLIBSS-SL and the OLIBSSST models . . . . . . . . . . . . . . . . . . . 32

6 Novel hybrid charging station. . . . . . . . . . . . . . . . . 41

7 The $\mathrm{M} / \mathrm{G} / \mathrm{s} / \infty$ queueing model. . . . . . . . . . . . . . 45

8 The flow chart of the metaheuristic for the OLIHCS-SL and the OLICHSST models . . . . . . . . . . . . . . . . . . 50

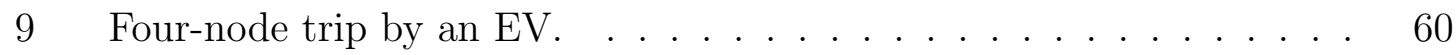

10 Total network-infrastructure cost as a function of $\gamma$. . . . . . . 64

11 Percent change in total network infrastructure cost as a function of $\gamma .66$

12 Aggregated arrival rate in stations for various $W_{\max }$ of Set $4 . \quad \ldots 67$

13 Total cost $(\times \$ 1,000)$ and number of stations as a function of $W_{\max }$. 68

14 Percent change in cost for the OLIBSS-ST model as a function of $W_{\max }$. 69

15 Percent change in battery inventory for the OLIBSS-ST model as a function of $W_{\max } . \quad \ldots \ldots \ldots \ldots$

16 Total cost as a function of $\beta$ and $\gamma$ of Set $4 \ldots \ldots \ldots \ldots$

17 Aggregated arrival rate in stations for various $W_{\max }$ of Set $4 \ldots \ldots 73$

18 Average number of batteries for various $W_{\max } \ldots \ldots \ldots \ldots$

19 Average number of superchargers as a function of $W_{\max }$. . . . . . 76

20 Results for Mid-Ohio case with the OLIHCS-SL model. . . . . . . 78

21 Results for Mid-Ohio case with the OLIHCS-SL model. . . . . . . 79 


\section{CHAPTER 1}

\section{INTRODUCTION}

Greenhouse-gas emissions from conventional internal-combustion-engine vehicles (ICEVs) make a major contribution to global climate change [1, 2]. One solution is the large-scale adoption of electric vehicles (EVs) [3], which offer numerous benefits. First, EVs are more eco-friendly because they significantly reduce greenhouse-gas emissions mainly carbon dioxide $\left(\mathrm{CO}_{2}\right)$ from ICEVs. EVs produce much less noise pollution and cost less to drive than ICEVs. In the U.S., the end-user cost of EVs is 2 to 3 cents per mile, compared with 13 cents per mile for ICEVs [2]. Third, EV batteries can potentially feed electricity into the grid during the peak-electricity-use time to help level the load of the power grid. This so-called "vehicle-to-grid" technology may reduce the number of new power plants required, thus saving generation costs and reducing the overall environmental footprint. Finally, EVs can help reduce the reliance of the U.S. on imported petroleum and thereby increase energy security.

On the other hand, several obstacles hinder the mass adoption of EVs. Unlike ICEVs, which can be refueled in a matter of minutes, recharging EVs requires more sophisticated equipment and usually takes a much longer time. Even more challenging is the driving range of a fully charged EV, which is shorter than that of an ICEV with a full tank. For example, the Nissan Leaf, which is the best-selling battery electric vehicle in the U.S., offers a range of only 150 to 226 miles with a full battery charge but requires between 0.75 to 35 hours to recharge, depending on the charging voltage [4]. Furthermore, the number of EV chargers in the public domain, such as parking lots, office buildings, shopping areas, and highway rest areas, is far less than the number of gas stations. Overall, slow 
charging speed, short driving range, and inadequate access to charging points constitute major obstacles to mass adoption of EVs.

To overcome these challenges, the Israeli company Better Place has proposed a new infrastructure scheme called a "battery-swap station" as a complimentary charging method. A battery-swap station (BSS) allows an EV to replace a depleted battery with a fully charged battery in a matter of minutes. The station then moves the depleted battery into a stockroom to charge it using a smoother and more eco-friendly current. In this business model, batteries are owned by the charging service provider and leased to end-users. Such a business model can help users avoid the risks associated with deteriorating battery performance and can easily detect battery defects [5]. It also lowers EV selling costs because the cost of a battery accounts for about one-third of the value of an EV [6]. In addition, the charging service provider can charge end-users based on battery use [2]. During the 2008 Summer Olympics, such a battery swapping system was implemented for 50 electric shuttle buses in Beijing. In the summer of 2013, Tesla re-demonstrated the BSS concept on a much larger scale than Battery Place [7]. At the demonstration event, the new Tesla BSS completed the battery-swap process in just $90 \mathrm{~s}$, even faster than the conventional gas refueling process.

Recognizing the benefits of battery swapping, this dissertation proposes the concept of a hybrid EV charging station that consists of two types of services: battery swapping and fast level-3 DC charging. Under this concept, the first choice for battery recharging is to swap the battery, and the second choice is fast DC charging (the second choice comes into play when the hybrid charging station is out of fully charged batteries). The advantages of such a hybrid charging facility include the guarantee of fully charged batteries and the fast overall recharging time, regardless of the recharging method used for a particular EV.

Although the battery swapping concept offers convenience to EV users, charging service providers must overcome some unprecedented challenges to make it a reality. The service provider must decide not only the BSS locations but also the number of batteries that each BSS should hold. In fact, Avci et al. [8] suggest that 
the number of batteries far exceeds the number of EVs deployed under some service level. Unfortunately, EV batteries are expensive, so for the BSS business model to work, battery inventory must be efficiently managed, with a high customer service level. In addition, travel patterns and demands vary from one region to another, so BSS locations must correlate with inventory decisions. Given these complexities involving battery swapping services and stations, we study herein new models that simultaneously consider BSS location, battery inventory (i.e., stock level for fully charged spare batteries),, and queueing for battery charging.

In particular, we study two types of BSSs. The first type offers only a battery swapping service, whereas the second type offers not only a battery swapping service but also fast on-site level-3 DC charging (a.k.a. "supercharging") as a complimentary service when the battery inventory is insufficient to swap out the batteries of incoming EVs. Two types of service-level constraints are considered. The first type considers the service level for stations by ensuring that the stockout probabilities of both batteries and superchargers do not exceed a certain threshold. The second type of service-level constraint ensures that the total sojourn time in EV stations does not exceed a pre-determined time. In all models, the objective is to minimize total cost, which comprises facility fixed costs and procurement costs for spare batteries for the first type of EV station, plus purchasing costs for superchargers for the second type of EV station.

This dissertation makes a three-fold contribution. First, it investigates a joint location and inventory allocation model with two types of battery services by using two criteria: service level and total sojourn time in stations. Under the assumption that in the near future, electric vehicle penetration rate will be much greater and there will be enough EVs that will cause charging delay, we use the Erlang loss and Erlang queueing models to study the operations of EV charging stations. Second, we consider two types of stations in real-world operations: a BSS that only offers a battery-swap service and a hybrid charging station that offers an on-site charging service in addition to a battery swapping service. The proposed location-inventory models achieve three performance goals: guaranteed state of charge (SOC) for all 
spare batteries, ensured customer service quality, and controlled load for the electricity grid. Third, because the joint battery-swap and supercharge service network models are mixed-integer program with nonlinear chance constraints, we develop metaheuristic algorithms that uses using Tabu search for efficient solutions. Extensive computational study provides valuable managerial insights for real-world EV charging services.

The remainder of this dissertation is organized as follows: Chapter 2 reviews the literature on estimate of EV charging demand, design of battery swapping station, facility location, and inventory control with service level. Chapter 3 describes two joint location and inventory models for the basic BSS system, and Chapter 4 presents the two models for the novel joint battery swapping and supercharing statation system. Finally, chapter 5reports the computational results, including a real-world case study, and Section 6 concludes the study. 


\section{CHAPTER 2}

\section{LITERATURE REVIEW}

We divide the literature on modeling and simulation of EV charging infrastructure into five categories: estimation of EV charging demand, design of infrastructure for BSSs, general facility location, inventory control with service-level constraints, and joint inventory and location models.

\subsection{Estimate of EV Charging Demand}

A proper estimate of EV charging demand is essential as input to design the EV charging infrastructure. Therefore, we begin by reviewing ways to estimate EV charging demand. Jung et al. [9] studied the problem of locating taxi charging stations in Seoul, South Korea. They divided the city into 560 centroids and randomly generated point-to-point (i.e., centroid-to-centroid) travel demand by using a spatially uniform distribution. They set the average EV range to 70 miles on highways and 80 miles in city traffic. Each EV started with a random initial SOC and obtained a recharge when the battery range was consumed. Arias et al. [10] used cluster analysis to classify different traffic patterns based on various factors such as vehicle type, battery technology, battery capacity, and initial battery SOC. By assuming that each EV starts to search for a charging service when its SOC reached $20 \%$, they converted traffic volume to charging demand over 24 hour periods. Hosseini et al. [11] considered the refueling-location problem and captured the EV flow on a network by using pre-defined origin-destination pairs. First, trips with origin-destination distances exceeding half of the battery range were included in the study. Second, an expended network with nodes along the trips was formed to catch EVs that could not finish their trip without charging under the given SOC 
assumptions. Xu et al. [12] collected data from nearly 500 private and commercial EV charging and driving activities from February 2011 to January 2013. The data include SOC (\%), GPS location, and the corresponding clock time for each trip or charging activity. Given these data, they calculated the average distance driven per day, the average distance between charges, the average number of charges per day, and the average SOC (\%). All these statistics were used in a mixed logit model that produced a charging-location preference for each EV trip. They then aggregated all trips by location to get the EV charging demand for each location. Majidpour et al. [13] collected charging activity from the customer end and from chargers at the University of California, Los Angeles campus from December 7, 2011 to February 28, 2014. To forecast the charging load, they use four machine learning techniques: time-weighted dot-product-based nearest neighbor, modified pattern sequence forecasting, support vector regression, and random forest. Of these four machine learning methods, modified pattern sequence forecasting had the lowest symmetric mean absolute percent error (6.28\%). Pevec et al. [14] studied EV charging demand by using business data from a charging-infrastructure provider in the Netherlands. The study clusters the existing charging stations into several charging zones. By using the number of places of interest, charging time, and charging day as independent variables and EV charging demands in each charging zone as the dependent variable, the study used multiple linear regression and XGboost to predict the charging demand of 1765 charging stations. The study concluded that the use of XGboost leads to better performance with a mean absolute error of 0.03.

\subsection{Design of Electric Vehicle battery swapping Station}

Many studies on BSSs focus on their impact on the existing power grid. Wang et al. [15] studied an optimization model to maximize the net income by determining the optimal BSS locations and sizes for EV charging power demand from three different customer types: the fixed customer, the habitual customer, and the random customer. They also used as service constraint the service radius of a single BSS, but did not include a service level of incoming EVs. Pan et al. [16] used 
a two-stage stochastic programming model to locate BSSs and obtain the number of batteries in each station to meet power demand. In Pan's model, the first-stage model minimizes setup cost and battery purchasing cost, whereas the second-stage model minimizes travel distance, unmet demand penalty cost for EV drivers, and the cost of generating and shedding loads for the power grid. However, this two-stage model considered EV charging demand as deterministic data. Finally, Widrick et al. [17] tackled a BSS management problem to determine the optimal policy for vehicle to grid and grid to vehicle to maximize the expected total profit over a fixed time.

Other studies focused on the operation and service for BSSs. For example, Sun et al. [18] studied the optimal strategy for battery purchasing and charging for BSSs by using the dynamic fluid model to approximate the stochastic demand. They also developed a two-stage operational model to study the capital cost associated with batteries, the cost of waiting in charging queues, weekly total demand, and BSS energy price. Sun et al. [19] used an expanded network to formulate arrival demand and calculate battery inventory and BSS location. Raviv [20] studied the scheduling problem for charging spare batteries in BSSs by using a continuous and $K$-piecewise linear function as a penalty function to optimize a combined measure of service level and cost. Their results show that the first-in-first-out policy for charging batteries generates fewer penalties for fixed charging capacity and time-varying price. In addition, to assign charging time and volume to batteries, they viewed the battery-charging process as a demand-supply model. Mak et al. [2] studied BSS-infrastructure planning by using paths and subpaths to model the EV charging demand. They also used a combination of the first-in-first-out policy and a fixed charging-time duration to replenish battery SOC in the model.

Service availability and queuing network for single BSSs have also been studied in the past. For example, Lu et al. [21] proposed an index called the "availability of battery swapping service per day," which is the percent of EVs that can charge their battery in a day. This index evaluates the serviceability of a single BSS. Tan et al. [7] simulated the charging process as a Markov chain and used the steady-state probability to determine the blocking probability. Similarly, Yudai and 
Osamu [22] modeled the battery recharging process in three stages: waiting, recharging, and fully charged. They then used the queueing theory to calculate the number of batteries that a station should hold. Avci et al. [23] studied the BSS business model and its pricing, adoption, management, and environmental impact. Finally, Jamian et al. [24] used an artificial-bee-colony algorithm to determine the optimal placement and sizing of battery swapping stations by minimizing the total power loss as the objective function.

Other studies used maximum flow capture of alternative-fuel vehicles to determine BSS size and location. Hodgson [25] introduced the flow-capturing location-allocation model in which the number of vehicles on a specific road is considered as origin-to-destination flow, and BSSs are located to capture maximize flow. Kuby and Lim [26] expand the flow capture model to make it suitable for alternative-fuel vehicles by considering vehicle range and possible remaining range. In Kuby's model, every origin-to-destination pair joins different nodes, which can be origin, intermediate, or destination nodes, and these nodes can form different combinations of site stations on the path that vehicles take to complete trips. The model first chooses optimal combinations of nodes for alternative-fuel vehicles of a given range, and then uses these combinations to locate stations to maximize trips that require refueling.

Unlike the maximum-flow-capturing model, Wang et al. [27] designed a facility-location model to reduce setup cost for fast-refueling stations such as BSSs. Instead of capturing maximum trip flow like Kuby's study, Wang et al. focused on the amount of fuel or power vehicles have left to calculate the refueling requirements at each node to reach the next node in trips. Armstrong [28] then extended Wang's model by introducing charging rate, charging time, and multiple types of charging stations. In Armstrong's study, instead of being fully charged, batteries need only be sufficiently recharged to reach the next node, which saves vehicle waiting time in stations. Furthermore, Armstrong's model considers multiple types of chargers with various charging rates and times. Lu et al. [29] also used Kuby's model as a basis and added queuing theory to limit the time vehicles have to wait in stations. They 
used captured flow as arrival rate and Little's law to determine the average waiting time in a station, and then used a genetic algorithm to locate stations.

Other studies focused on how EV-driver preference impacts BSS location. Sweda and Klabjan [30] used an agent-based simulation model to strategically deploy charging stations by identifying charging-station coverage, driver inconvenience, and driver vehicle preference. Finally, Chen et al. [31] developed a mixed-integer model to minimize the walking distance between charging stations while satisfying demand in Seattle, USA.

\subsection{Facility Location}

Given that determining optimal locations for BSSs is an example of the classic facility-location problem, we now review the literature on location models. Erlebacher \& Meller [32] used a continuous inventory-location model in a rectangular region. The study first determined the optimal number of distribution centers by using the Karush-Kuhn-Tucker condition and then calculated the location of distribution centers by applying a heuristic algorithm to determine the upper and lower bound of inventory and facilities cost.

More closely related to our models are the facility-location problems with service level constraint. We first review works on the optimal facility-location problem with type-I inventory-service-level constraints. Note that, when integrating service level, most location models become nonlinear integer programming problems, which are often solved by applying Lagrangian decomposition. Miranda et al. [33] studied warehouse location in response to stochastic demand for a given service level. They modeled customer demand as a normal random variable, used type-I service level as a parameter to determine the safety stock level, and finally used Lagrangian decomposition to solve the model. Sourirajan et al. [34] considered the lead time consisting of three parts: waiting to be shipped, shipping, and unloading. They also used Lagrangian decomposition to compute warehouse location and to assign warehouses to customers. Aghezzaf [35] studied warehouse location with uncertain demands by first developing a location model with fixed 
demand, and then extending the model to different uncertain-demand scenarios (but without considering demand distribution). Finally, Aghezzaf solved the model by applying Lagrangian decomposition and robust optimization. Nozick [36] introduced two Lagrangian-decomposition-based heuristics: allocations and decoupling relaxations. By dualizing allocation and coupling constraints on the location and allocation variables, respectively, Nozick constructed lower bounds at each iteration of Lagrangian relaxation. After obtaining the upper bounds via a greedy heuristic, both relaxation methods yielded satisfactory gaps between the lower and upper bounds. When solving for a nonlinear service constraint, many studies have used linear approximations. For example, Nozick et al. [37] modeled service level more explicitly when the probability of demand exceeded inventory, and they used linear regression to linearize the probability function, which was integrated into the warehouse-location model. Candas \& Kutanoglu [38] linearized a nonlinear time-based service constraint in their model by tabulating potential fill rates for demand and stock levels at the first stage, following which they introduced other binary variables to apply a table look-up process.

Some publications directly address the variance of stochastic demand in the facility-location problem. For example, Daskin et al. [39] designed a location-inventory model to minimize the sum of facility setup cost, inventory transportation cost, and holding cost. The model assumes normal random demand and uses the $(Q, r)$ policy to calculate the inventory each facility should hold during the order lead time. By assuming a fixed variance-to-mean ratio, the model reduces the number of nonlinear terms and assigns demand nodes to the facilities that are opened in the given nodes. Shen et al. [40] developed a location risk-pooling model. In this model, some retailers serve as distribution centers and hold inventory calculated by using the economic order quantity model. They reconstructed the model as a set-covering model and discussed solutions to two cases: a constant variance-to-mean ratio and zero variance for demand. Romeijn et al. [41] studied a single-red distribution-center multi-retailer system that took optimal replenishment intervals of both distribution centers and retailers into 
consideration in terms of inventory holding cost and used standard deviation of demand to obtain safety stock. However, they did not consider inventory cost in transportation (i.e., pipeline cost).

Some papers considered the multi-echelon inventory-location problem. Mak \& Shen [42] studied the two-echelon inventory-location problem and addressed not only the location of distribution centers but also inventory levels at plants and in-transit inventory holding cost. By getting an upper bound for in-plant inventory and fixing inventory in distribution to possible values in each iteration, the study used Lagrangian procedures to obtain an optimal customer assignment. You \& Grossmann [43] studied the design of integrated multi-echelon supply chains. They proposed "net lead time," which is defined as the difference between the replenishment lead time of a node, and then guaranteed a service time to its successor node. Like many similar studies, the random demand is modeled as a normal random variable, and safety stock is calculated based on the standard deviation.

Simulation is another approach for location-inventory models. Ridlehoover [44] used Monte Carlo simulation and risk analysis to solve the facility-location problem. He used a $p$-median model to choose 30 locations out of 297 candidate locations to minimize the total weighted travel distance of customers in the first stage. In the second stage, he used four types of distributions: normal, uniform, custom, and lognormal to simulate four characteristics of facilities' annual worth: initial investment, annual cost, annual benefit, and interest rates, respectively. Based on the result of the simulation, he chose facility locations in descending order of revenue received to get maximum annual worth. Fu [45] outlined computer simulation methods and software for quantitative variables and parameters in a supply chain field. Lim et al. [46] studied supply chain replenishment policies by using a simulation approach in which a mathematical model is first used to obtain facility capacities by minimizing supply chain costs. They then consider four replenishment policies: build to order, build to plan, continuous, and periodic. By comparing total operational cost from the simulation of each replenishment with the 
results of an analysis of variance, they conclude that a periodic policy is the best policy.

Service level is another factor to consider when planning location and allocation. Karratas \& Yak [47] studied the problem of locating emergency service systems with a backup service level. The study used the $p$-median location problem to allocate the backup facility first, and then used a discrete event simulation model to maximize facility use by reducing customer travel and wait time.

Petrovic et al. [48], however, considered uncertainty not only from customers but also from the supply side. The study models a serial link of all facilities in the supply chain and sets demand with upper and lower bounds based on experience and subjective judgment from managers rather than on a probability distribution from past records. By increasing the safety stock level, the model compensates for uncertainty due to external suppliers.

\subsection{Inventory Control with Service Level}

BSS services require that adequate spare batteries be in stock. When an EV swaps a battery on site, batteries held at the swapping station are either fully or near fully charged and ready for swapping. Because batteries take a significant amount of time to charge, sufficient charged batteries must be ready while depleted batteries are being charged. This operation is similar to inventory control in the production and service industries that ensures sufficient stock to ensure a certain service level. Thus, we review in this section the studies on inventory control.

In the inventory-control literature, many have argued that back-order cost is not a good measure to capture all consequential costs caused by inventory shortage (see, e.g., Ouyang et al. [49]), so service-level constraint has been considered by many researchers. The literature review in this section also considers inventory-control policies with service-level constraints. Aardal et al. [50] used the $(Q, r)$ stock-control system and calculated the order time points and lot sizes simultaneously with a service-level constraint to study the relationship between shortage cost and service level. The paper presents two different measurements of 
service: the fraction of demand covered from stock and the average number of shortages per year.

Some researchers studied type-II service level (i.e., fill rate) for inventory control. Heijden [51] studied multi-echelon divergent network inventory with fill-rate constraints. Assuming all demand is satisfied only at the end stock points, he developed an optimization model to determine optimal order-up-to-level inventory, mean inventory, and time-averaged inventory at both stock points and in the pipeline. He used a Gamma distribution to approximate the relationship between the order-up-to-inventory level and the fill rate of each stock point. Finally, he used a heuristic algorithm to find a near-optimal solution for the multi-echelon inventory network. Sculli \& Wu [52] studied stock control with two suppliers and normal lead times. They created a table of mean and standard deviations with numerical integration for the normally distributed lead time during which the next order will arrive.

Janssens et al. [53] used the expected shortage per replenishment cycle (ESPRC), which is the expectation value of the difference between demand and safety stock, as the service level in the linear programming for an inventory management decision problem. The study mainly focused on minimizing safety stock with a certain ESPRC constraint, whereas information about demand (i.e., the demand distribution) in the lead time was unknown. They first used the claim-size distribution proposed by Goovaerts [54] to get the upper and lower bounds for the demand parameters and then used the new vendor model to obtain the desired inventory policy. Cole [55] used the same service-level measurement to design a production-distribution network. The study used a piecewise linear system to approximate the nonlinear relationship between the safety factor, which is part of the safety stock calculation, and the expected amount of the unit normal variable that exceeds the safety factor. 


\subsection{Joint Inventory-location Model}

The fifth category of the relevant literature involves the joint inventory-location model. Shahabi et al. [56] studied this problem for warehouses and plants. Their study not only models the daily demand and the demand for product lead time as a normal distribution but also considers the correlation coefficient between daily demands of certain products at different retailers and uses probability of stockout as a service-level measurement. Tapia-Ubeda et al. [57] studied the inventory-location problem with stochastic inventory constraints. Their study considered transportation costs between warehouses and customers and used a Benders decomposition to solve the nonlinear and non-convex mathematical model. Dai et al. [58] used fuzzy constraints to define the service level for perishable items and implemented a genetic algorithm plus a hybrid harmony search to obtain the optimal location and inventory for warehouses. Puga et al. [59] considered a two-level supply chain network and used different lead times between central plants and distribution centers and between distribution centers and retailers. Due to the nonlinearity and non-convexity of the formulation, the study proposes a heuristic algorithm to determine the facility locations. Amrir-Aref et al. [60] studied the multi-sourcing inventory-location problem by splitting it into two stages. The first stage determines the distribution locations by maximizing the expected revenue, whereas the second stage used the linear approximation of the $(s, S)$ policy to obtain the optimal inventory of distribution centers over months. Diabat et al. [61] studied the joint inventory-location problem by considering consumption demand as a Markov transition process and calculated the expected amount of reorders, lost sales, and inventory by using Markov queueing theory. The study used simulated annealing and a direct search method to solve the problem. Zheng et al. [62] integrated item routing into the inventory-location problem and used generalized Benders decomposition to reduce the problem dimensions and reduce computational time. Hiassat et al. [63] studied the location-inventory-routing problem with perishable products by considering the perish time period and used a genetic 
algorithm to determine the warehouse location, inventory, and routing plan that minimized total cost.

\subsection{Summary of Literature}

Although the battery-swap service for charging EVs is a relatively new field, it shares many similarities with operations for facility location, demand allocation, and inventory control with a service level. Therefore, existing research in these areas can be useful. Many previous studies also focused on the unique operations associated with BSSs, including their impact on the grid, battery charging scheduling, EV-adoption patterns, or queueing models for a single station. In the supply chain and inventory control area, studies have considered how inventory control affects service levels. By defining different service levels and lead time in the supply chain, many studies furnish optimal assignments for components in the supply chain at minimal cost while maintaining service level. The model we propose herein integrates queueing theories into the supply chain area, which means that the service provider can stock the minimal number of batteries required to cover a given

percent of incoming demand, with each battery having the desired SOC (e.g., 80\%). 


\section{CHAPTER 3}

\section{JOINT FACILITY LOCATION AND INVENTORY MODELS FOR BATTERY SWAPPING STATION SERVICES}

\subsection{Introduction}

In this chapter, we consider the joint location and inventory models for BSS services for EVs. BSS locations and the stock of fully charged batteries at these locations will be determined by the pre-defined service level and the total sojourn time (i.e., service time plus waiting time) in the BSS queue, if any.

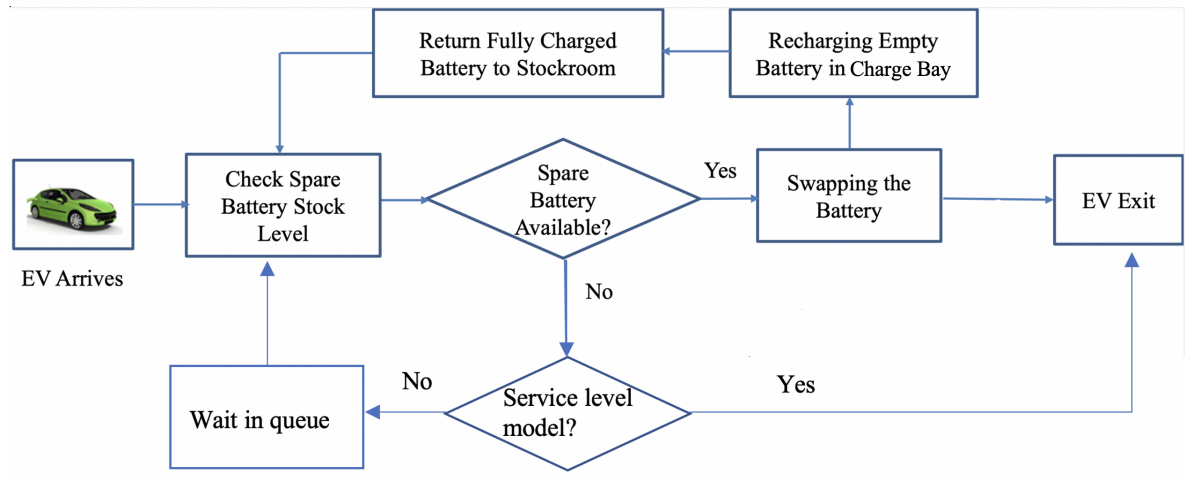

Figure 1. Battery swapping station with priority queue.

Figure 1 shows a flow chart describing how an EV is serviced at a BSS. Given an incoming EV, the BSS first checks that a spare battery with sufficient SOC is in stock and ready to swap. If so, the batteries are swapped. The depleted battery is then charged in the charging bay and then deposited in the stockroom to be swapped into a subsequent EV. After swapping batteries, the current EV exits the BSS.

If no spare battery is available to swap, the BSS blocks the EV directly or asks the EV to wait until a battery is available to swap, depends on whether there is 
a waiting area.

Although typical inventory control analysis is used for operational purposes, the strategic nature of the decision means that, in this dissertation, the inventory of spare batteries with desired SOC serves to plan the capacity of BSSs in a given charging network. Thus, our intent is not to make the tactical or operational-level inventory decisions but rather to minimize the total cost, which consists of the fixed operating cost for a BSS and the cost of purchasing batteries. We consider in particular a network of BSSs, in contrast with some publications that consider single BSSs (see, e.g., Ref. [7]). The model also considers the service-level constraint or the total sojourn time constraint, whereby we specify the probability of stockout or total sojourn time for an EV to obtain a fully charged battery within a pre-specified threshold time for the entire network of BSSs.

The problem at hand is closely related to the classic facility-location model (see, e.g., Ref. [64]), whereby a facility is opened in a candidate location only when the location has positive activity. In this study, we open a BSS in candidate locations where demand exists for fully charged batteries. The aspect of capacity planning in the proposed model will determine the proper number of fully charged batteries needed at open locations.

As mentioned previously, the existing literature on designing BSSs or EV-charging infrastructure does not consider waiting queues and associated queuing characteristics for EVs when making location decisions. The model proposed herein uses stochastic queuing theory (assuming Poisson arrival and exponential service time) and the Erlang loss function [65] to calculate the steady-state stockout probability for fully charged batteries at BSS locations. It also uses the Erlang queueing function [65] to calculate the stead-state total sojourn time at BSS locations. These constraints determine, in part, the optimal battery capacities for BSSs. Furthermore, including such service-level or total service time constraints also ensures that all batteries charged or swapped at these BSSs have a SOC at above a pre-determined threshold level (e.g., 80\% SOC).

The two joint inventory-location models with the service-level constraint and 
with the total sojourn time constraint are presented in the subsequent sections in this chapter.

\subsection{Research Contribution}

Considerable research efforts have been devoted over the last five years to the areas of battery swapping and charging stations. However, most location models for designing the BSS network infrastructure do not incorporate a queueing model or the SOC for decisions involving battery-inventory purchasing. As a consequence, the number of customers lost because of insufficient SOC-ready batteries, which has a huge impact on service quality, has not been studied. Nevertheless, in other domains, queueing has been proven to affect the location decision. For example, Wang et al. [66] studied how customer waiting time affects the location decisions for automatic teller machines. Brandeau et al. [67] studied how stochastic queueing affects the location decisions for facilities in a plane with rectilinear distances. In

the present study, we consider unique factors for queueing at BSSs, such as charging time, SOC, the probability of rejecting or queueing customers, etc. and build a location model accordingly.

This research contributes to the design of EV-charging infrastructure in the following two areas:

1. We develop two integrated BSS inventory-location models. The first model requires each BSS to have enough batteries with at least $80 \%$ SOC; otherwise, the EV leaves the station. The second model incorporates a pre-determined maximum sojourn time that an EV can spend for a battery swap. In both models, we combine the inventory-location model with queueing theory to gain insight into the operational services of a BSS. The goal is to determine the number of batteries required at each BSS and the locations of the BSSs.

2. We use the Erlang function as a nonlinear constraint in both models mentioned above. Decision variables are embedded in both power and factorial functions, which make it challenging for applications to large-scale problems. 
Heuristic algorithms are developed for the models, and two heuristic methods provide an original means to incorporate the Erlang function into an optimization model in other applications.

\subsection{Notation}

We use the following notation in both proposed models:

TABLE 1. Sets and indices.

$j \in J \quad$ Set of potential locations for BSSs

$i \in I \quad$ Set of EV traffic analysis zone (TAZ) demands for BSSs

TABLE 2. Parameters and indices.

$\lambda_{i} \quad \mathrm{EV}$ arrival rate in TAZ $i$

$F_{j} \quad$ Setup cost of candidate charging stations $j$

$\mu_{b} \quad$ Battery recharge rate in the charging bay

$F_{b} \quad$ Purchase cost of per battery

$\gamma$ Overall allowable stockout probability

$W_{\max }$ Maximum allowable sojourn time in a BSS

$\tau$ Battery swapping time in a BSS

$\theta_{i} \quad$ Demand rate during lead time of batteries charging in TAZ $i$

$P_{j} \quad$ Power capacity at candidate location $j$

$P_{b} \quad$ Power required for charging a battery in charging bay

$d_{i j} \quad$ A binary parameter equals 1 if customer zone $i$ is in a certain battery range of candidate location $j$ or 0 otherwise

TABLE 3. Decision variables. 
$x_{j}$ Whether location $j$ is chosen

$s_{j} \quad$ Base stock level of battery inventory at station $j$

$\theta_{j} \quad$ EV arrival rate at station $j$ during lead time for charging batteries

$y_{i j} \quad$ Binary variable equal to 1 if customer $i$ is assigned to candidate location $j$

$\lambda_{j} \quad$ Aggregate arrival rate at station $j$

$B_{j} \quad$ Probability of battery stockout at station $j$

$C_{j} \quad$ Probability of queueing at station $j$

3.4 Modeling EV Arrival and Battery Charging Service Using M/G/s/s

This section explains our approach for modeling the arrival pattern for EVs and the battery charging process as per the $\mathrm{M} / \mathrm{G} / \mathrm{s} / \mathrm{s}$ model. The arrival of EVs in stations is assumed to follow a Poisson distribution, whereas the battery-charging process follows a general distribution.

\subsubsection{Model and data analysis}

We consider the following process for EVs arriving at a single BSS. Each EV arrives at random at the BSS and obtains an immediate battery swap only if spare batteries with the minimum-required SOC (e.g., 80\%) are available. To appropriately model arrival and charging service distribution, we make several important assumptions:

- Arrivals: The arrival process involves two assumptions: First, EV arrivals at each BSS occur independently. Second, the arrival rate is constant, so the average time between arrivals is the inverse of the arrival rate. In essence, these two assumptions allow us to model the arrival process as a Poisson process, as has is widely done in the literature (see, e.g., Tan et al. [7] and Mak et al. [2]).

- Charging process: The charging process is assumed to follow a general distribution. The charging process is defined as charging a battery from empty to the desired SOC, which can require anywhere from a half-hour to over a day [4], depending on the desired SOC. Many studies use different distributions for the 
charging process $[2,68]$. In this study, we model the charging process as a general distribution. In reality, charging time may have significant variance because the process of unloading batteries from incoming EVs differ for different EVs, which prevents the charging time from being described by a specific distribution.

Consequently, the general distribution is judged to be the best way to describe the charging process.

- Battery inventory: In this model, battery inventory is a decision variable that is subject to a service-level constraint. As described previously, an incoming EV is serviced only if a battery is available with desired SOC; otherwise, the EV is blocked by the BSS or has to queue, depending on the business model. Therefore, the capacity of a BSS is determined by its battery inventory.

Given these assumptions, the operations of a BSS may be modeled as an $\mathrm{M} / \mathrm{G} / \mathrm{s} / \mathrm{s}$ queueing model. Figure 2 shows a transition diagram for the $\mathrm{M} / \mathrm{G} / \mathrm{s} / \mathrm{s}$ queueing model. This model is a special case of the $\mathrm{M} / \mathrm{G} / \mathrm{K}$ queueing model, where arrivals are Markovian and service time follows a general distribution. The M/G/K model accurately predicts the number of customers in a service queueing service and has been applied in a variety of fields with similar queueing patterns, including single-warehouse inventory control [69], single-server systems [70], and clinical planning [71].

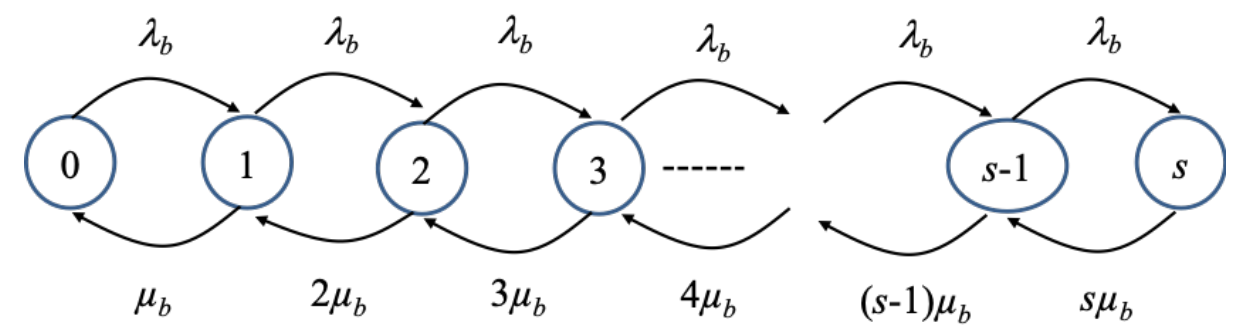

Figure 2. The $\mathrm{M} / \mathrm{G} / \mathrm{s} / \mathrm{s}$ queueing model.

\subsubsection{The Erlang loss model}

The service level of a BSS is defined as the probability that the inventory of batteries with at least $80 \%$ SOC can meet the demand of arriving EVs. Therefore, 
the battery service time in a BSS is the time required to charge a battery to $80 \%$ SOC. The service level in station $j$ is estimated based on the availability of spare batteries upon the arrival of an EV. In the literature, the Erlang loss model gives a closed formula for calculating the stockout probability:

$$
B\left(\theta_{j}, s_{j}\right)=\operatorname{Pr}\left\{O_{j}>s_{j}\right\}=\frac{\theta_{j}^{s_{j}} / s_{j} !}{\sum_{k=0}^{s_{j}}\left(\theta_{j}^{k} / k !\right)}
$$

where $\theta_{j}$ is the demand for spare batteries (i.e., the arrival rate) at station $j$ during the service time, and $s_{j}$ is the base stock level of spare batteries at station $j$. Here, service time is defined as the time interval from when the empty battery is removed from the EV to when said battery is charged to at least $80 \%$ SOC. The function describes, as a function of time, the probability that incoming demand $O_{j}$ exceeds inventory $s_{j}$. Let $N_{b}$ be the number of batteries recharging in the charging bay. The expectation of $N_{b}$ can be estimated as

$$
\left.E\left[N_{b}\right]=\sum_{k=0}^{s} k \operatorname{Pr}\left\{N_{b}=k\right\}=\frac{\lambda_{b}}{\mu_{b}}\left(1-\operatorname{Pr}\left\{N_{b}=s\right\}\right]\right)=\theta(1-B(s)) .
$$

For a given level of charge, $E\left[N_{b}\right]$ allows us to determine the average power required for the charging bay.

The Erlang loss function was first formulated as an $\mathrm{M} / \mathrm{M} / \mathrm{s} / 0$ queue by Erlang [72] in 1917 to address the probability of the number of busy lines in telephone trunk groups. Because the model is insensitive, it is also used in $\mathrm{M} / \mathrm{G} / \mathrm{s} / \mathrm{s}$ model $[73,74]$. The Erlang loss function has also been used to predict the number of customers lost, such as to estimate the probability of losing patients in an emergency department [75], for deploying ambulances [76], designing an operating theater and intensive-care unit [77], etc. However, the Erlang loss function has been used as a testing or evaluation tool rather than as a decision function. In the present research, however, the Erlang loss function is contained in the model. In this study, determining the number of batteries a BSS should hold for a certain service level is closely related to issues of resource capacity in service systems, such as server centers, in which service levels are determined by the number of servers a center is allocated $[78,79]$. 
Before using the Erlang loss function, we introduce its important properties.

Lemma 1. To ensure numerical stability, the Erlang loss function can be calculated by its inverse; namely,

$$
B\left(\theta_{j}, s_{j}\right)= \begin{cases}1, & s_{j}=0 \\ \frac{1}{1+\frac{\theta_{j}}{s_{j}} \frac{1}{B\left(\theta_{j}, s_{j}-1\right)}}, & s_{j}=1,2,3, \ldots\end{cases}
$$

Proof. (See Ref. [80].) To show the recursion for the reciprocal, we denote the reciprocal the Erlang loss function as $R(\theta, s)=\frac{1}{B(\theta, s)}$ and $S(s)=\sum_{k=0}^{k=s} \theta^{k} / k !$. Then,

$$
R(s)=\frac{S(s)}{\theta^{s} / s !}=\frac{S(s-1)+\theta^{s} / s !}{\theta^{s} / s !}=\frac{s R(s-1, \theta)}{\theta}+1 .
$$

Lemma 2. $B(\theta, s)$ increases monotonically with $\theta$.

Proof. To prove that the Erlang loss function increases with $\theta$, we first take the partial derivative with respect to $\theta$ :

$$
\frac{\partial B(\theta, s)}{\partial \theta}=B(\theta, s)\left[\frac{s}{\theta}-1+B(\theta, s)\right] .
$$

By Little's law, we get the number $E N$ of $\mathrm{EV}$ in the system:

$$
E N=\theta(1-B(\theta, s))
$$

Furthermore, because we block all waiting EVs out of the system, the available inventory is always greater than or equal to the number of EVs in the system: $s \geq E N$. Thus, we have $B(\theta, s) \geq \max \left\{0,1-\frac{s}{\theta}\right\}$, so $\frac{\partial B(\theta, s)}{\partial \theta} \geq 0$.

Lemma 3. $B(\theta, s)$ decreases monotonically with $s$.

Proof. To show that the Erlang function decreases with $s$, we have

$$
B(\theta, s-1)-B(\theta, s)=B(s-1)\{1-\theta /[s+\theta B(\theta, s-1)]\}>0
$$

because

$$
B(\theta, s-1) \geq \max \{0,1-(s-1) / \theta\} \geq \max \{0,1-s / \theta\} .
$$


These three lemmas of the Erlang loss function are crucial for solving our models. Lemma 1 offers a practical means of computing the Erlang loss function by avoiding calculation of the factorial function, which poses a computational challenge when $s>170$. Conversely, Lemmas 2 and 3 show the relationship between arrival rate, battery base stock level, and service level, which we use to develop our algorithms.

\subsubsection{The Erlang queueing model}

We also consider another BSS service model, which allows EVs to wait for battery charging if no battery with the desired SOC is available for immediate swapping. In this business model, we want to ensure that the sojourn time of EVs in BSSs is shorter than a pre-determined value. To determine the sojourn time in a BSS, we first calculate the probability that EVs are obliged to wait in a BSS. According to the Erlang queueing model, this probability can be calculated as follows:

$$
C\left(\theta_{j}, s_{j}\right)=\frac{\frac{\theta_{j}^{s}}{s !\left(1-\theta_{j} / s\right)}}{\sum_{k=0}^{s-1} \frac{\theta_{j}^{k}}{k !}+\frac{\theta_{j}^{s}}{s !\left(1-\theta_{j} / s\right)}}, \quad \text { if } s_{j}>\theta_{j} .
$$

The time that an EV spends in a BSS is the sum of the waiting time $W_{q}$ and the actual battery swapping time $\tau$. The waiting time $W_{q}$ can be calculated as

$$
W_{q}=\frac{1}{\mu_{b}\left(s_{j}-\theta_{j}\right)} .
$$

Therefore, the EV sojourn time in a BSS is

$$
W=\frac{C_{j}\left(s_{j}\right)}{\mu_{b}\left(s_{j}-\theta_{j}\right)}+\tau .
$$

The expected number of batteries being charged can be expressed as

$$
E N=\theta .
$$

Similarly to the Erlang loss function, we now introduce the important properties of the Erlang queueing function: 
Lemma 4. The Erlang queueing function can be calculated by the inverse function of the Erlang loss function; namely,

$$
C(\theta, s)=\frac{1}{R(\theta, s)-R(\theta, s-1)}
$$

See Ref. [80].

Lemma 5. $C(\theta, s)$ increases monotonically with $\theta$. See Ref. [80].

Lemma 6. $C(\theta, s)$ decreases monotonically with s. See Ref. [80].

These lemmas will help us solve the models containing the Erlang queueing functions.

\subsection{The Mathematical Models}

We design two models for BSS services. The first model considers the optimal BSS location and battery inventory problem subject to service-level constraints. In this model, an EV can obtain an immediate battery swap if a battery with the desired SOC is available; otherwise, the EV is blocked by the BSS. In other words, there is no waiting area. This model is called "optimal location and inventory for BSS with service-level constraints" (OLIBSS-SL). The second model considers the optimal BSS location and battery-inventory problem subject to the total sojourn-time constraint. In this model, an EV can queue if no spare battery is available for an immediate swap. This model is called the "optimal location and inventory model for BSS with service time constraint" (OLIBSS-ST).

\subsubsection{Optimal location and inventory for BSS with service-level constraints}

We consider the OLIBSS-SL model as a coverage model consisting of a set of candidate locations and a set of traffic-analysis zones (TAZs). Without loss of generality, we assume that customers are willing to use a BSS only if the customer-BSS distance is less than a certain percentage of the battery drive range. We also assume that in the near future EV penetration rate will be high enough 
that there will be delays for charging service. Therefore, Poisson process can be used to model the EV charging demand arrival. Let $I$ be the number of TAZs and $J$ be the number of candidate BSS locations. A binary parameter $d_{i j}$ is introduced to indicate whether a BSS is w ithin a pre-defined battery range of customer sites for $i=1,2, \ldots, I$, and $j=1,2, \ldots, J$. Each EV arrives at a BSS according to the Poisson process, and the arrivals are independent. In this model, we determine where BSSs should be placed, which TAZs are assigned to a station, and how many spare batteries should be held at an opened station. The goal is to minimize the setup cost for opening stations and the battery-procurement cost subject to a stockout probability requirement. The OLIBSS-SL model is formulated as follows:

$$
\begin{aligned}
\text { (OLIBSS-SL }) \min & \sum_{j=1}^{J}\left(F_{j} x_{j}+F_{b} s_{j}\right), \\
\text { s.t. } & \sum_{j} y_{i j}=1 \quad \forall i, \\
& y_{i j} \leq d_{i j} x_{j} \quad \forall i, j, \\
& \theta_{j}=\lambda_{j} / \mu_{b} \quad \forall j, \\
& \lambda_{j}=\sum_{i} y_{i j} \lambda_{i} \quad \forall j, \\
& B_{j}\left(s_{j}\right) \leq \gamma \quad \forall j, \\
& B_{j}\left(s_{j}\right)=\frac{\theta_{j}^{s_{j}} / s_{j} !}{s_{j}}\left(\theta_{j}^{k} / k !\right) \\
& P_{b} \theta_{j}\left(1-B_{j}\left(s_{j}\right)\right) \leq P_{j} \quad \forall j, \\
& s_{j} \leq M x_{j} \quad \forall j, \\
& s_{j} \in \mathbb{Z}^{+} \quad \forall j, \\
& x_{j} \in\{0,1\} \quad \forall j, \\
& y_{i j} \in\{0,1\} \quad \forall j .
\end{aligned}
$$

In the OLIBSS-SL model, the objective function (1) serves to minimize the total setup and battery cost. Constraint (2) states that each TAZ must be covered by one station. Constraint (3) sets the maximum allowed distance (e.g., $10 \%$ of the driving 
range of an EV) from a TAZ to an assigned BSS. Constraint (4) calculates the expected EV arrivals at station $j$ during a battery-recharge cycle in the charging bay. Constraint (5) aggregates the EV arrival rate at a BSS over all assigned TAZs. Constraint (6) defines the service-level requirement (i.e., the battery stockout probability), which cannot exceed a pre-specified level (i.e., $\gamma=0.05$ ). Constraint (7) is the Erlang loss formula for the stockout probability. Constraint (8) states that the power use at any station should not exceed grid capacity. Constraint (9) ensures that no battery is placed at a station unless the station is open. Constraints (10)-(12) specify the decision-variable types for $s, x_{j}$, and $y_{i j}$, respectively. In Section 3.6, we propose a metaheuristic algorithm to efficiently solve this model.

3.5.2 Optimal location and inventory model for battery swapping station with service-time constraint

The OLIBSS-ST model only involves the battery swapping service. Instead of a probability-based service-level constraint, this model requires that the total 
sojourn time for an EV at a station not exceed a pre-defined threshold:

$$
\begin{aligned}
\text { (OLIBSS-ST) } \min & \sum_{j=1}^{J}\left(F_{j} x_{j}+F_{b} s_{j}\right), \\
\text { s.t. } & \sum_{j} y_{i j}=1 \quad \forall i, \\
& y_{i j} \leq d_{i j} x_{j} \quad \forall i, j, \\
& \theta_{j}=\lambda_{j} / \mu_{b} \quad \forall j, \\
& \lambda_{j}=\sum_{i} y_{i j} \lambda_{i} \quad \forall j, \quad \forall j \quad \forall j, \\
& \frac{C_{j}\left(s_{j}\right)}{\mu_{b}\left(s_{j}-\theta_{j}\right)}+\tau \leq W_{\max } \quad \forall j, \\
& \frac{\theta_{j}^{s}}{s !\left(1-\theta_{j} / s\right)} \quad \forall-C_{j}\left(s_{j}\right) \quad \forall j, \\
& \sum_{k=0} \frac{\theta_{j}^{k}}{k !}+\frac{\theta_{j}^{s}}{s !\left(1-\theta_{j} / s\right)} \\
& P_{b} \theta_{j} \leq P_{j} \quad \forall j, \\
& s_{j} \leq M x_{j} \quad \forall j, \\
& s_{j} \in \mathbb{Z}^{+} \quad \forall j, \\
& x_{j} \in\{0,1\} \quad \forall j, \\
& y_{i j} \in\{0,1\} \quad \forall j .
\end{aligned}
$$

In the OLIBSS-ST model, constraint (18) defines the total sojourn time requirement, which cannot exceed a pre-specified level (i.e., $W_{\max }=15$ minutes). Constraint (19) is the Erlang queueing formula for the queueing probability.

Constraint (20) states that the power consumption at any station should not exceed grid capacity. All other constraints and the objective function are the same as for the OLIBSS-SL model.

\subsection{Solution Approaches}

The Erlang function is computationally challenging because the decision variable $s_{j}$ is embedded in the power and factorial functions. In fact, many off-the-shelf solvers generally cannot compute values for such highly nonlinear 
functions, let alone provide a global optimal solution for the optimization problem. Therefore, for these two models, we first study the mathematical properties of the problem, namely, the relationship between the arrival rate, the inventory necessary, the stockout probability, and the queueing probability. Subsequently, we develop heuristic methods to obtain quality solutions for the models.

\subsubsection{Relationship between $s_{j}$ and $\theta_{j}$}

As discussed previously, the OLIBSS-SL model has a constraint to ensure that the stockout probability does not exceed a threshold value, while the OLIBSS-ST model ensures that the total sojourn time does not exceed a threshold value. Note that both the stockout probability and the total sojourn time are monotonically decreasing functions of the battery inventory $s$. Consequently, given an open BSS, the total battery cost is minimized by storing the minimum number of batteries to satisfy the threshold value for either model. The results show that

- for a given stockout probability or a given maximum sojourn time, the number of batteries needed increases essentially linearly with EV arrival rate.

We validate the above observation by using a two-step procedure. First, for a given stockout probability $\gamma$, we calculate the required battery inventory $s(\theta)$ by incrementing $s$ in steps of 1 until $B(s)<\gamma$. Second, we perform a regression analysis between the variable $\theta$ and the resulting $s(\theta)$ obtained in the first step. Figure 3 shows that the desired number of batteries increases almost nearly with the arrival rate for the Erlang loss function, and Figure 4 shows that the desired number of batteries increases nearly linearly with arrival rate for the Erlang queueing function. Tables 4 and 5 reveal that the linear relationships have a rather high $R^{2}$, thus validating our observation. This observation is significant because it not only simplifies the nonlinear chance constraint but also leads to the following theorem and our subsequent heuristic algorithms.

TABLE 4. Results of linear regression test for Erlang loss function. 


\begin{tabular}{|c|c|c|c|c|}
\hline$\gamma$ & Intercept & Slope & $p$-value & adj $R^{2}$ \\
\hline 0.01 & 20.84 & 1.00681 & 0.0001 & 1 \\
\hline 0.1 & 7.21524 & 0.9014 & 0.0001 & 1 \\
\hline 0.2 & 4.04018 & 0.8003 & 0.0001 & 1 \\
\hline 0.3 & 2.64934 & 0.70016 & 0.0001 & 1 \\
\hline
\end{tabular}

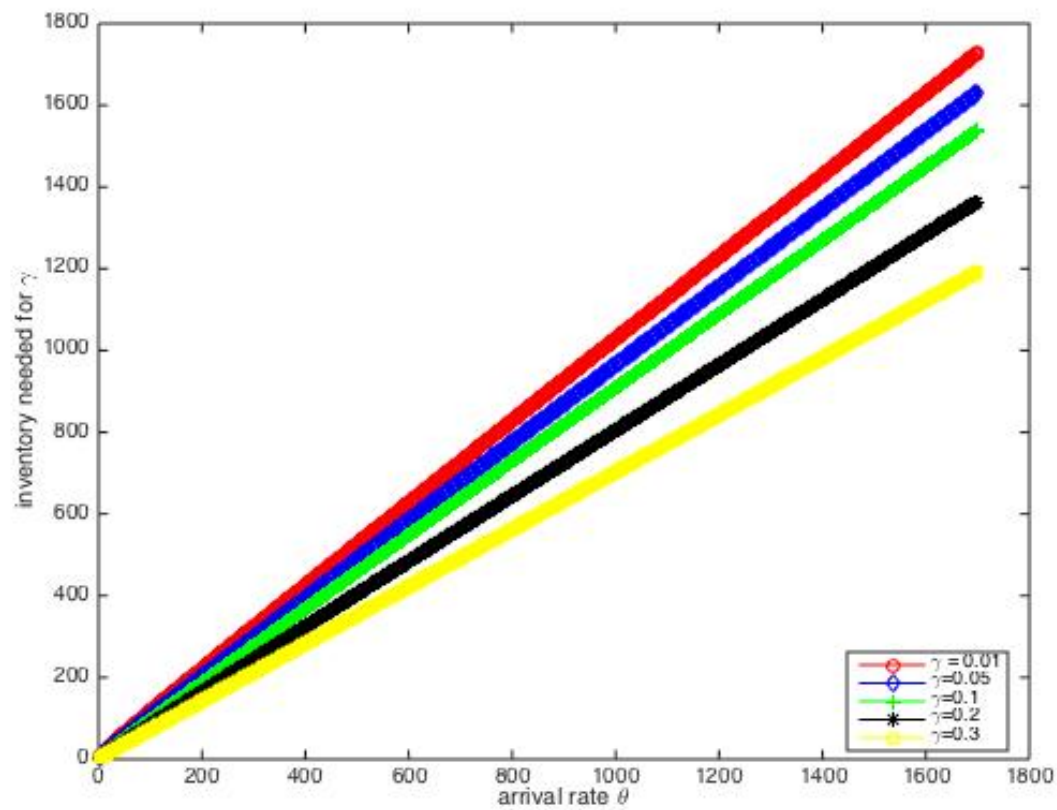

Figure 3. Inventory required for different arrival rates for target $\gamma$.

TABLE 5. Results of linear regression test for Erlang queueing function.

\begin{tabular}{|c|c|c|c|c|}
\hline$W_{\max }$ & Intercept & Slope & $p$-value & adj $R^{2}$ \\
\hline $10 \mathrm{~min}$ & 6.676 & 1.043 & 0.0001 & 1 \\
\hline $15 \mathrm{~min}$ & 5.519 & 1.028 & 0.0001 & 1 \\
\hline $20 \mathrm{~min}$ & 4.822 & 1.021 & 0.0001 & 1 \\
\hline $25 \mathrm{~min}$ & 4.245 & 1.017 & 0.0001 & 1 \\
\hline $30 \mathrm{~min}$ & 3.977 & 1.013 & 0.0001 & 1 \\
\hline
\end{tabular}




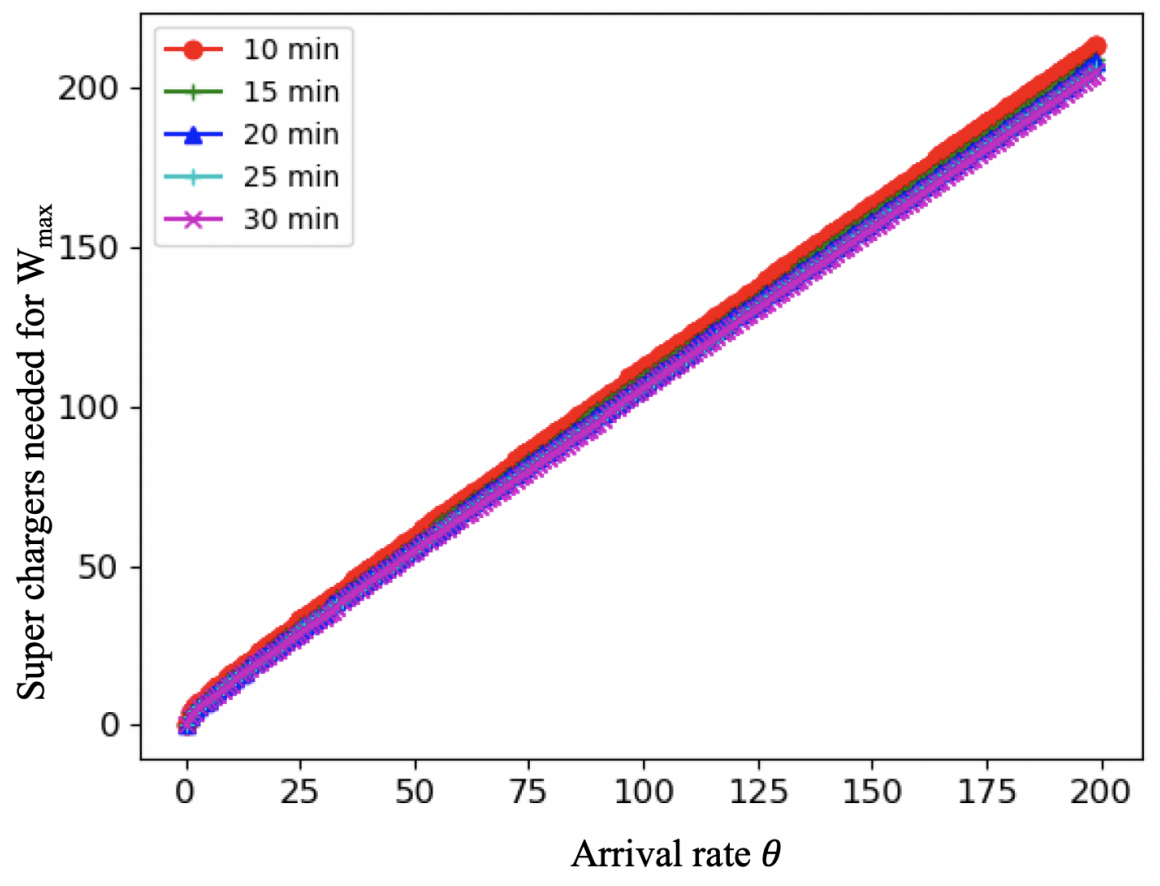

Figure 4. Inventory required for different arrival rates for target $W_{\max }$.

Theorem 1. The optimal location and allocation strategy assigns EV customers to an existing BSS first before opening a new BSS.

Proof. The linear relationship between $s$ and $\theta$ can be written as $s=a \theta+b$. We consider two feasible strategies: The first strategy assigns all $I$ EV charging-demand coverage areas to a single BSS with the total cost of $z_{1}=F_{1}+F_{b}\left(\sum_{i=1}^{I} a \theta_{i}+b\right)$.

The second strategy assigns $m$ coverage areas to one BSS and assigns the remaining $I-m$ coverage areas to a second BSS. Thus, its associated cost is $z_{2}=F_{1}+F_{b}\left(\sum_{i=1}^{m} a \theta_{i}+b\right)+F_{2}+F_{b}\left(\sum_{i=m+1}^{I} a \theta_{i}+b\right)$. Hence, we have $z_{1}-z_{2}=F_{1}+F_{b}\left(\sum_{i}^{I} a \theta_{i}+b\right)-\left[F_{1}+F_{b}\left(\sum_{i=1}^{m} a \theta_{i}+b\right)+F_{2}+F_{b}\left(\sum_{i=m+1}^{I} a \theta_{i}+b\right)\right] \leq 0$.

This indicates that $z_{1} \leq z_{2}$, so the first strategy is optimal.

Given the optimal customer allocation policy, the next step is to determine which candidate locations should be assigned a BSS. 
The objective function in the model has two terms: setup cost and battery-purchasing cost. We design a metaheuristic algorithm containing subroutine algorithms (1) through (4) to solve for a quality solution. Figure 5 shows the flow chart of the overall metaheuristic

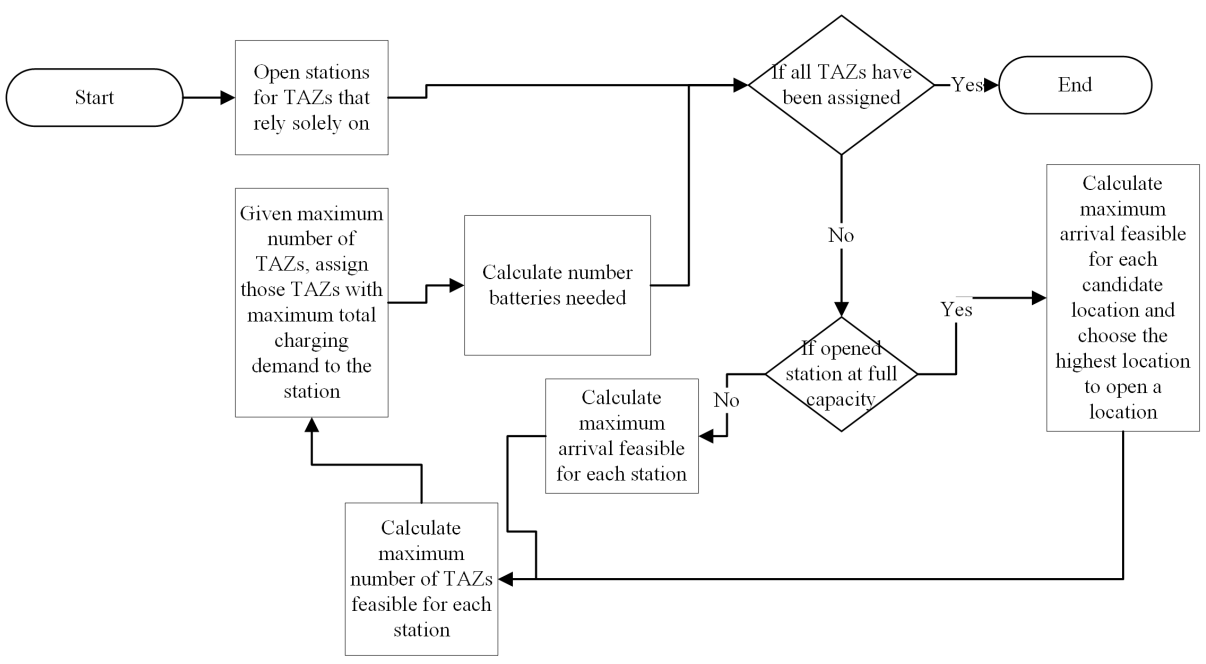

Figure 5. The flow chart of the metaheuristic for the OLIBSS-SL and the OLIBSS-ST models

\subsubsection{A metaheuristic algorithm for the OLIBSS-SL and the OLIBSS-ST models}

Given the optimal number of batteries for each location, we determine the optimal location-allocation plan by following the principles of the location-covering model. To maintain the desired service level or sojourn-time level, an important step in the BSS location-allocation decision is to convert the power capacity to the number of EVs that a station can handle. For a given service-level threshold and a power capacity $P_{j}$ at station $j$, the process of converting the power capacity to the allowable EV arrival rate for the models is given in Algorithm 1. In both models, based on Lemmas 3 and 6, the algorithm increases the number of spare batteries until the desired service-level or sojourn-time constraint is satisfied. 
$\overline{\text { Algorithm } 1 \bar{\lambda}_{j}=\operatorname{Max} \operatorname{Arrival}\left(P_{j}, \gamma, \text { or } W_{\max }\right) / / \text { the maximal rate of } \mathrm{EV} \text { arrivals }}$ feasible at station $j$

INITIALIZE $P=0$, and $\bar{\lambda}_{j}=\mu_{b}$

If it is the OLIBSS-SL model then

repeat

a. increase $s$ (from $s=1$ ) until $B\left(\frac{\overline{\lambda_{j}}}{\mu_{d}}, s\right) \leq \gamma$ using Eq. (7)

b. Update $P$ by using constraint (8) for the OLIBSS-SL model or (20) for the OLIBSS-ST model

c. $\overline{\lambda_{j}}=\overline{\lambda_{j}}+\mu_{b}$

until $P \geq P_{j}$

else if it is the OLIBSS-ST model

$\overline{\lambda_{j}}=\left\lfloor\frac{P_{j}}{P_{b}}\right\rfloor$

return $\overline{\lambda_{j}}$

By using the maximal arrive rate $\overline{\lambda_{j}}$ for each candidate station, as determined by Algorithm 1, we compute the maximum number $N_{j}$ of TAZs that can be assigned to station $j$ because constraint (2) requires all TAZs be covered. To minimize the total setup cost, the optimal solution should open the fewest possible stations, with each serving the maximum possible number of TAZs. Algorithm 2 below first sorts all TAZs that can be covered by station $j$ in ascending order of their EV arrival rates $\lambda_{j}$. It then chooses the first $N_{j}$ TAZs from the sorted list until reaching the maximum arrival rate $\overline{\lambda_{j}}$; thus, $N_{j}$ is returned as the maximum number of zones that station $j$ can cover. Note that we use the sorted list to determine $N_{j}$, but not the final assignment of TAZs ( $N_{j}$ or fewer) to station $j$. The latter is determined by Algorithm 3 . 


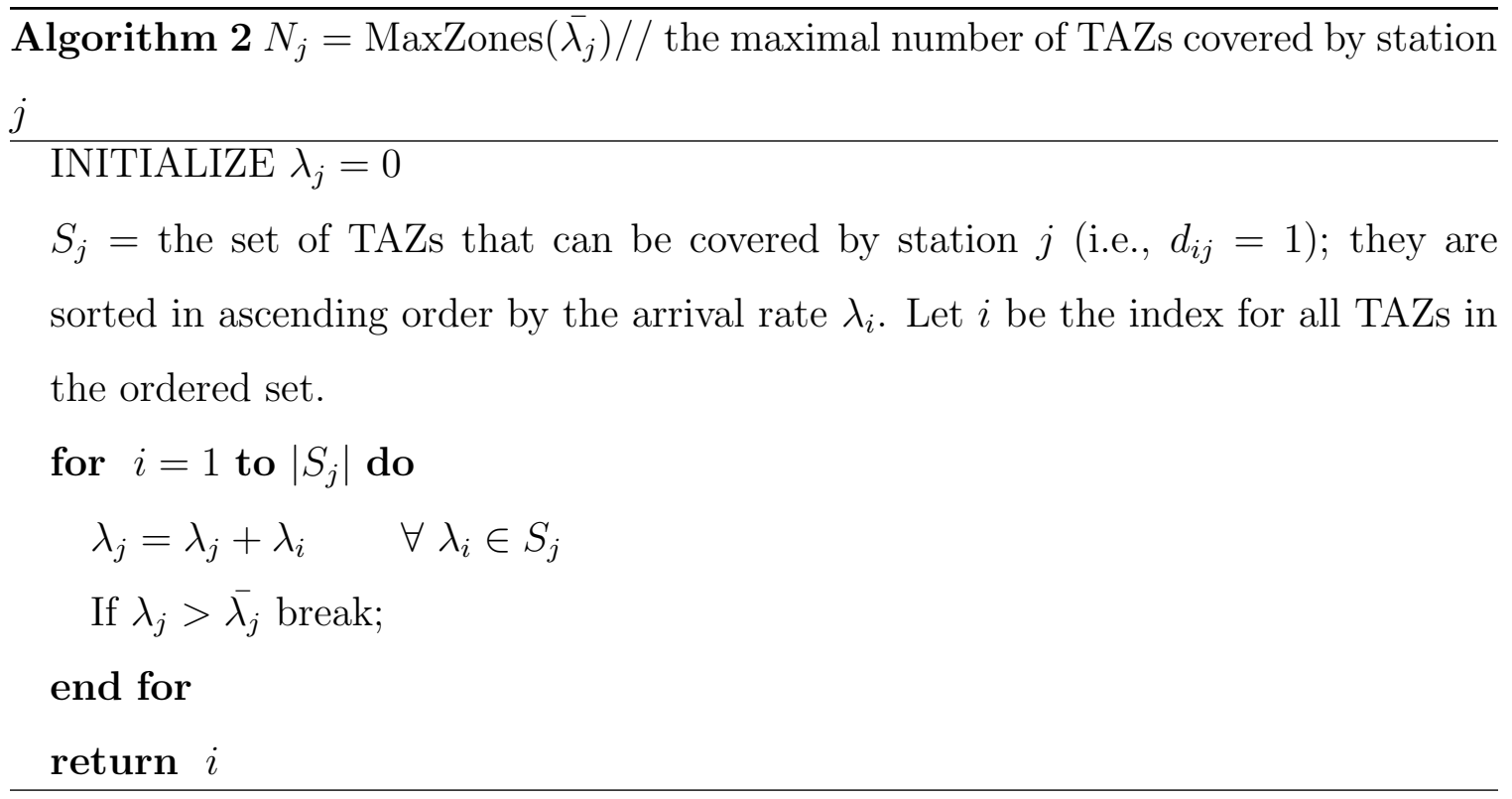

By using the maximum number of TAZs that station $j$ can cover, Algorithm 3 actually allocates the TAZs to the station by enumerating all combinations of $N_{j}$ TAZs and choosing the one that yields the maximum arrival rate for the station.

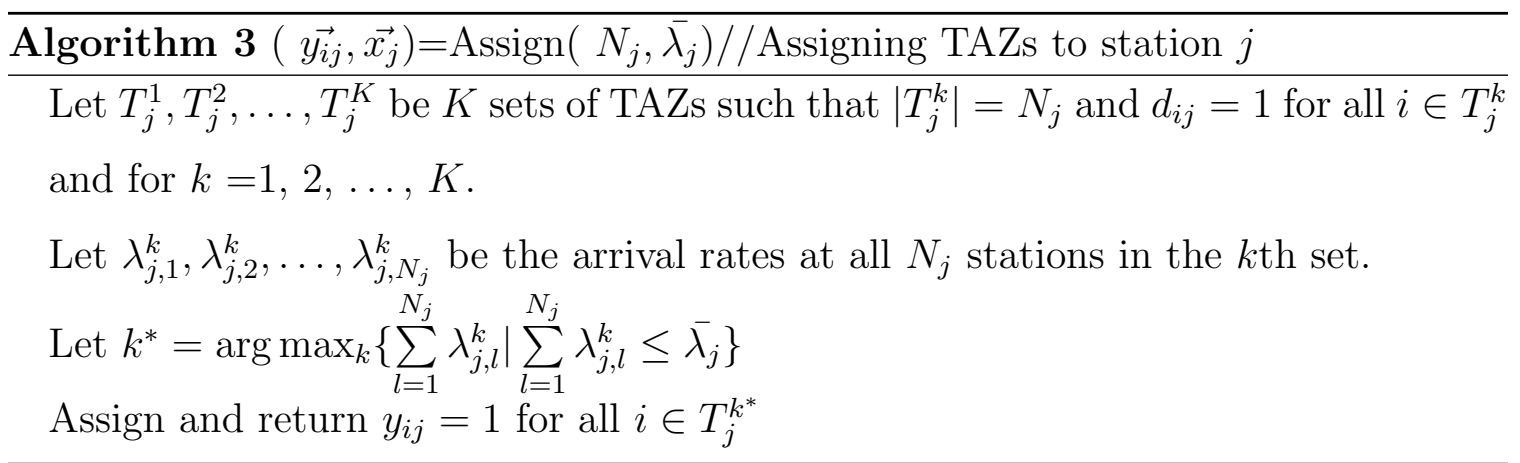

Once the assignment of TAZs to a candidate station $j$ is completed, the allocation of batteries is obtained by Algorithm 4. As mentioned previously, to minimize the objective function, the least number of batteries is preferred provided the service-level or sojourn-time constraint is satisfied. 


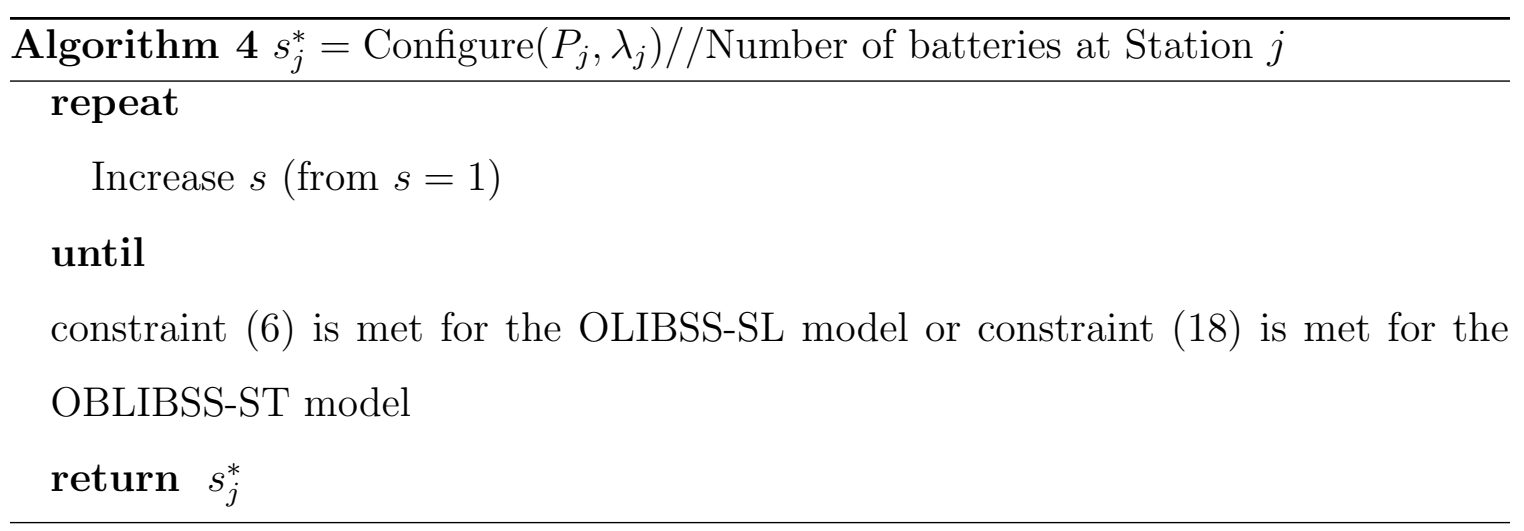

Algorithm 5 below integrates Algorithms 1-4 to provide a heuristic solution to models. Particularly, Algorithm 5 first uses Algorithms 1 and 2 to calculate the maximum number of TAZs that a candidate station is capable of covering, then uses Algorithm 3 to assign TAZs to all opened stations and finally determines the number of spare batteries for each station. 


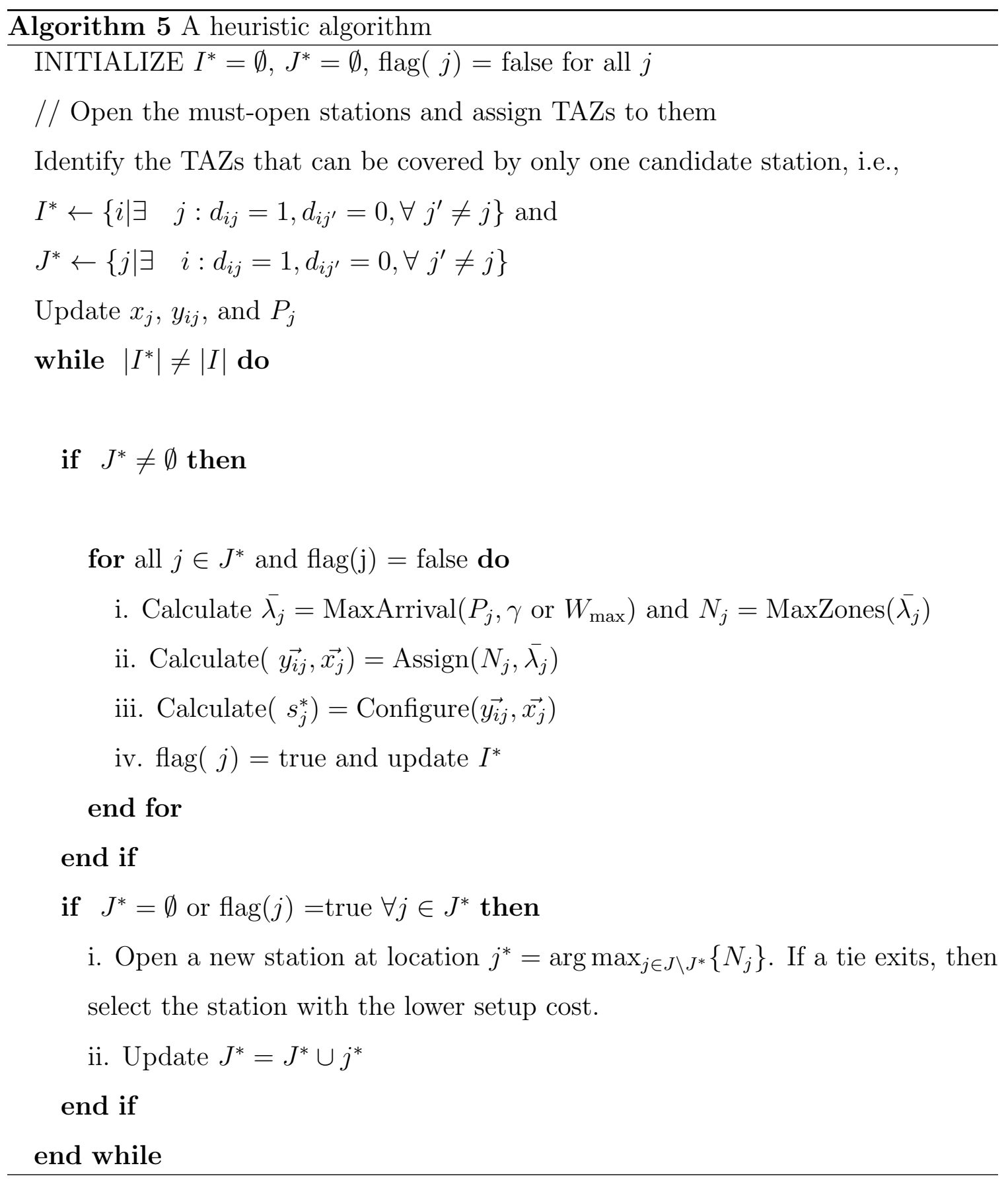

Since the Erlang loss constraint and the Erlang queuing constraint are nonlinear, we use a Tabu search to further improve the solution from Algorithm 5. In particular, the Tabu search in Algorithm 6 reassigns TAZs to different stations with the promise of potentially closing some stations, thus minimizing the total cost. The Tabu search, first created by Glover [81], is a metaheuristic search 
method that, to escape from local optima, finds the best solution in a defined neighborhood at every iteration. A Tabu list is used to track all historical solutions for higher efficiency. In particular, we consider the following two types of neighborhood functions:

- Substitution: Randomly select two unopened candidate stations. If the total setup cost of the two stations is less than that of an opened station, then open these two stations and re-assign TAZs to them and to the other opened stations. This neighborhood function is motivated by the fact that the priority in Algorithm 5 is given to stations' with a maximum number $N_{j}$ of zones, not to the setup cost.

- Consolidation: Randomly select a number of TAZs covered by a currently opened station $j$. Move the TAZs to another opened station provided the power capacity is not violated. This neighborhood function can reduce the number of batteries required in station $j$ and possibly the total cost.

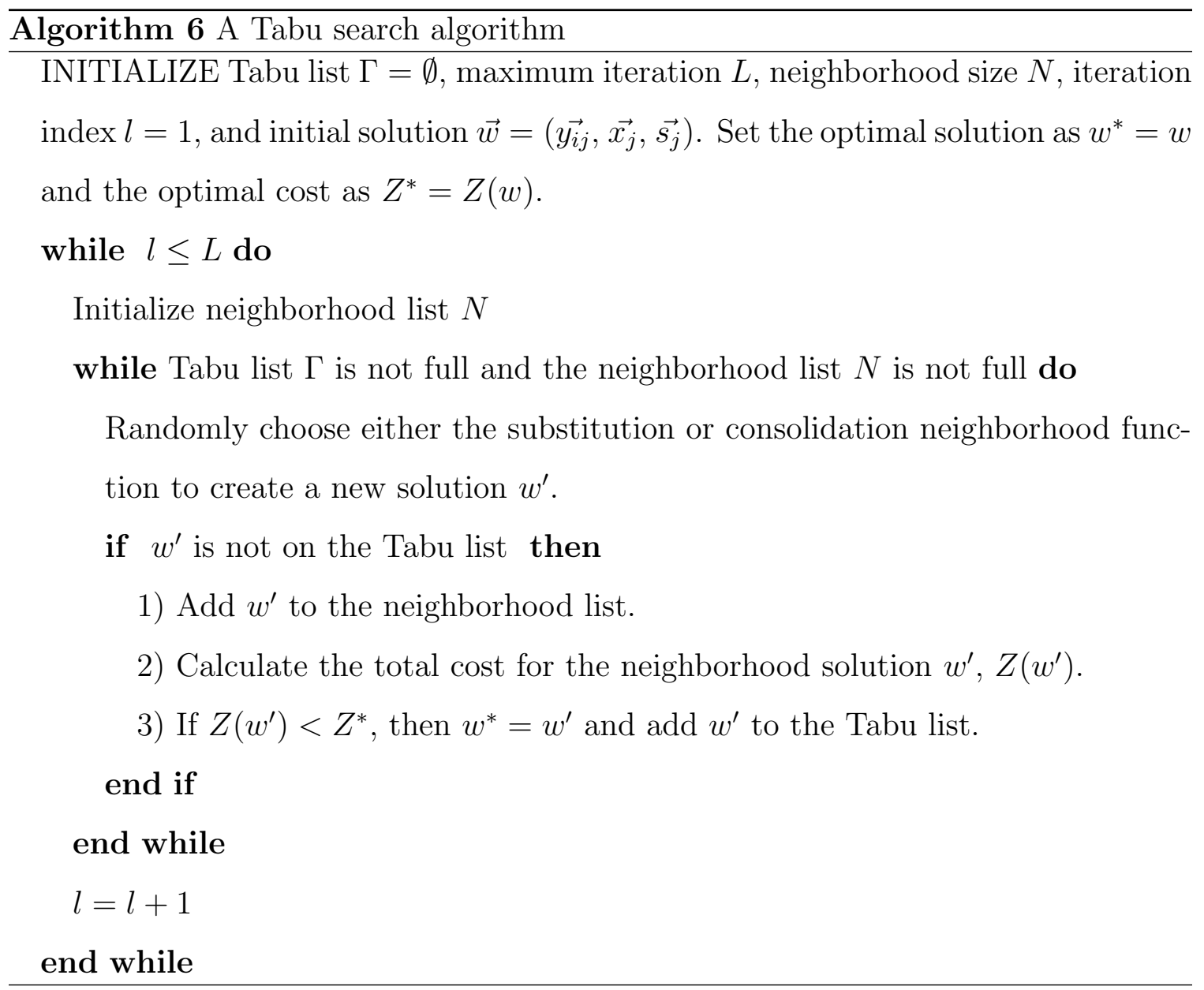




\subsection{An Illustrative Example for the Metaheuristic Method}

We now implement the algorithm for the OLIBSSL model by using an example with six TAZs and three candidate-station locations. The data in Table 6 include information on $d_{i j}$, the power capacity and setup cost for each candidate station, and the EV arrival rate in each TAZ. We set $\gamma=0.2$ in the numerical experiment. First, we use Algorithm 1 to calculate the maximum EV arrivals each candidate location can potentially serve. As a result, $\overline{\lambda_{1}}=21.875, \overline{\lambda_{2}}=20.31$, and $\bar{\lambda}_{4}=25$. Next, we apply Algorithms 2 and 3 to calculate the maximum number of TAZs covered by each station and further find that Station 1 covers four TAZs: 1, 3, 4, and 6; Station 2 covers three TAZs: 2, 4, and 6; and Station 3 covers three TAZs: 2, 3, 6, and 1. Since Stations 1 and 3 can serve three TAZs whereas Station 1 has a lower setup cost, we opt to open Station 1 first. Subsequently, we assign TAZs 1, 3, 4, and 6 to Station 1. Next, we need to cover TAZs 2 and 5. Realizing that both Stations 2 and 3 can cover TAZs 2 and 5, but that Station 3 has lower setup cost, we open Station 3 as the second service facility. After the assignment is completed, we further determine the optimal quantity of batteries. Station 1 is expected to serve 18 EVs per hour; therefore, by Lemmas 1 and 2, $s_{1}=61$ batteries with the total cost of $\$ 427000$ and total power of $576.94 \mathrm{~kW}$, which corresponds to $\gamma=0.19$. The same process is repeated for Station 3. After applying the Tabu search we obtain the final solution, where Station 1 has 61 batteries and Station 3 has 58 batteries, and the total network cost is $\$ 1.58$ million.

TABLE 6. Data for TAZs and candidate locations. 


\begin{tabular}{ccccc}
\hline TAZ/station & $j=1$ & $j=2$ & $j=3$ & $\lambda_{i}(\mathrm{EV} /$ hour $)$ \\
\hline$i=1$ & 1 & 0 & 1 & 6 \\
$i=2$ & 0 & 1 & 1 & 8 \\
$i=3$ & 1 & 0 & 1 & 3 \\
$i=4$ & 1 & 1 & 0 & 4 \\
$i=5$ & 0 & 1 & 1 & 9 \\
$i=6$ & 1 & 1 & 1 & 5 \\
\hline Power capacity $P_{j}(\mathrm{~kW})$ & 700 & 650 & 800 & \\
Setup cost $F_{j}(\times \$ 1,000)$ & 300 & 500 & 450 & \\
\hline
\end{tabular}




\section{CHAPTER 4}

\section{JOINT FACILITY LOCATION AND INVENTORY MODELS FOR NOVEL HYBRID CHARGING STATION}

\subsection{Introduction}

This chapter considers the joint location and inventory models for novel hybrid charging station (HCS) services for EVs. Decisions regarding the location of

HCSs and the stock of fully charged spare batteries and the number of superchargers at these locations is determined based on the pre-defined service level and total sojourn time (service time plus waiting time) in the HCS queue (if a queue exists).

The second business model has a supercharging area where empty batteries are charged very rapidly. In this model, the EV tries to obtain an immediate battery swap for a charged battery; otherwise it avails itself of the onsite supercharging service. Therefore, each EV leaves the station either with a swapped battery or a battery charged by a supercharger. Figure 6 shows the business model, which can be treated as a two-stage priority queueing network that combines a swapping queue and a supercharging queue. We developed two mathematical models: The first model has service-level constraints that are specified as the probability of stockout and queueing for the battery swapping and supercharging services, respectively. The second model has a pre-defined sojourn time for each EV in the station, regardless of whether the battery swapping or supercharging service constraint is active. 


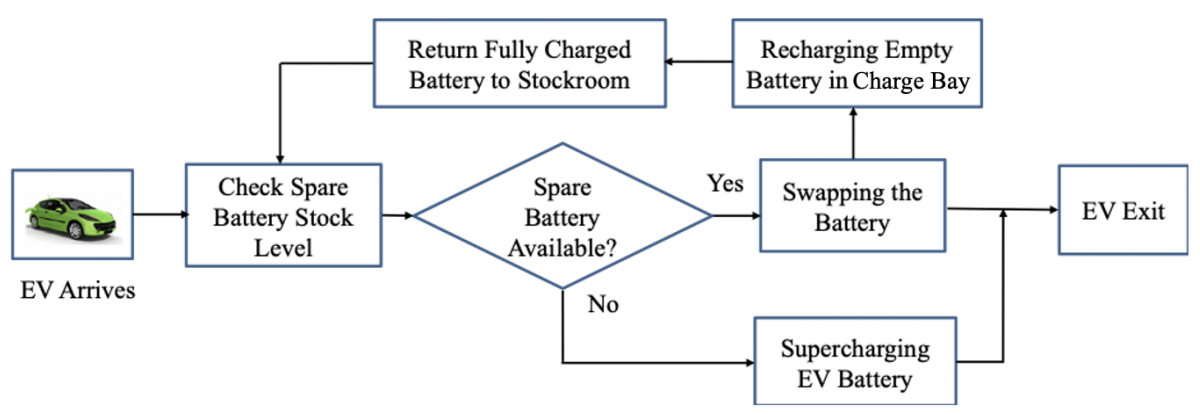

Figure 6. Novel hybrid charging station.

Figure 6 shows a flow chart describing how an EV is serviced at a HCS. When a HCS has an incoming EV, it first checks if a spare battery with sufficient SOC is in stock and ready to swap. If so, the batteries are swapped. The depleted battery will be charged in the charging bay and moved to the stockroom when fully charged, making it ready to swap for the next incoming EV. The EV that has finished swapping batteries then exits the BSS. If no spare battery is available to swap, the $\mathrm{EV}$ is sent to the on-site supercharger to receive the rapid-charging service.

\subsection{Research Contribution}

This model takes an early step to model and design an EV service network that offers both battery swapping and supercharging services. The study takes into account the demand uncertainty, range anxiety, service-level commitment, sojourn time, and electricity-grid constraints. We jointly optimize the station location, the stock of spare batteries, and the number of superchargers in an established station. The objective is to minimize the overall cost of the EV service infrastructure, which is comprised of the facility infrastructure, battery inventory, and supercharger installation.

We developed two models to tackle two types of desired services. The first model considers two different service levels for the network. For the battery-swap process, we use the battery stockout probability, whereas, for the supercharging process, we use the probability of having to wait for an available supercharger. The 
second model considers the total sojourn time for EVs in stations. The contributions of this paper are summarized as follows:

1. To the best of our knowledge, this research constitutes the first effort to investigate a joint location- and inventory-allocation model with two types of battery service. A station offering both battery swap and fast onboard charging is more realistic because an EV driver can use an alternative energy solution in case of battery stockout.

2. The operation of a HCS station is characterized as a two-stage Erlang priority queue with the battery-swap step preceding the supercharging step. Thus, the proposed planning model achieves three performance goals: guaranteed SOC for all spare batteries, ensured customer service quality, and controlled load to the electricity grid.

3. Since the proposed planning model is a nonlinear and mixed-integer optimization model, we develop a metaheuristic algorithm using a Tabu search to solve it efficiently. An extensive computational study provides valuable managerial insights for managing real-world EV charging and service operations.

\subsection{Notation}

We use the following notation in both proposed models:

TABLE 7. Sets and indices.

\begin{tabular}{ll}
$j \in J$ & Set of potential locations for BSSs \\
$i \in I$ & Set of TAZ demand for BSSs \\
\hline
\end{tabular}

TABLE 8. Parameters and indices. 
$\lambda_{i} \quad$ EV arrival rate in TAZ $i$

$F_{j} \quad$ Setup cost of candidate charging station $j$

$\mu_{b} \quad$ Battery recharge rate in charging bay

$\mu_{d} \quad$ Battery recharge rate in supercharger

$F_{b} \quad$ Purchase cost per battery

$F_{d} \quad$ Purchase cost per supercharger

$\gamma$ Overall allowable stockout probability

$\beta \quad$ Overall allowable queueing probability

$W_{\max }$ Maximum allowable sojourn time in BSS

$\tau$ battery swapping time in BSS

$\theta_{i} \quad$ Demand rate during lead time for battery charging in TAZ $i$

$P_{j} \quad$ Power capacity at candidate location $j$

$P_{b} \quad$ Power required for charging a battery in charging bay

$P_{d} \quad$ Power required for charging a battery using supercharger

$d_{i j} \quad$ Binary parameter equal to 1 if customer zone $i$ is in a certain battery range of candidate location $j$ or equal to 0 otherwise

TABLE 9. Decision variables.

$x_{j}$ Whether location $j$ is chosen.

$s_{j}$ Base stock level of battery inventory at station $j$.

$m_{j} \quad$ Number of superchargers in station $j$.

$\theta_{j} \quad$ Arrival rate at station $j$ during lead time for battery charging.

$\phi_{j} \quad$ Expected EV arrivals during a supercharging cycle.

$y_{i j} \quad$ Binary variable equal to 1 if customer $i$ is assigned to candidate location $j$

$\lambda_{j} \quad$ Aggregate arrival rate at station $j$

$B_{j} \quad$ Probability of battery stockout at station $j$

$C_{j} \quad$ Probability of queueing at station $j$.

$W_{j} \quad$ Total sojourn time at station $j$. 


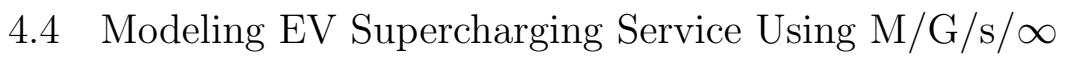

In this section, we find the appropriate data distribution to describe the EV arrival pattern and queueing model for the EV queueing pattern in the station. Specifically, we describe the battery swapping process by using an M/G/s/s model and use the Erlang loss function to describe the process. The on-site supercharging service is described by using an $\mathrm{M} / \mathrm{G} / \mathrm{s} / \infty$ model and we use the Erlang queue function to describe the process. We also study how the power consumption of these two charging processes impacts the power grid.

\subsubsection{Model and data analysis}

We consider the following EV arrival scenario for a single HCS: EVs randomly arrive at the given HCS and obtain an immediate battery swap only if spare batteries with a minimum SOC (e.g., 80\%) are available; otherwise, the EV goes to the on-site supercharging service to recharge its battery. In addition to the assumptions given in Section 3.4.1, we make another important assumption for supercharging service:

Supercharging queue. The arriving EV is directed to the supercharging queue if the stockroom has no spare battery. A supercharger uses the fast level-3 DC charging technology with an output power of up to $80 \mathrm{~kW}$. Let $\lambda_{d}$ be the EV arrival rate at the supercharging queue, then

$$
\lambda_{d}=\lambda_{j} B_{j}
$$

This result is due to the fact that all EVs blocked in the swapping queue move to the superchargers. Since $0<B(s) \leq 1$, we have $\lambda_{d} \leq \lambda_{b}$. Let $m$ be the number of installed superchargers in the station. The supercharging process can be modeled as an $\mathrm{M} / \mathrm{G} / \mathrm{s} / \infty$ queue, and the transition diagram is given in Figure 7 . 


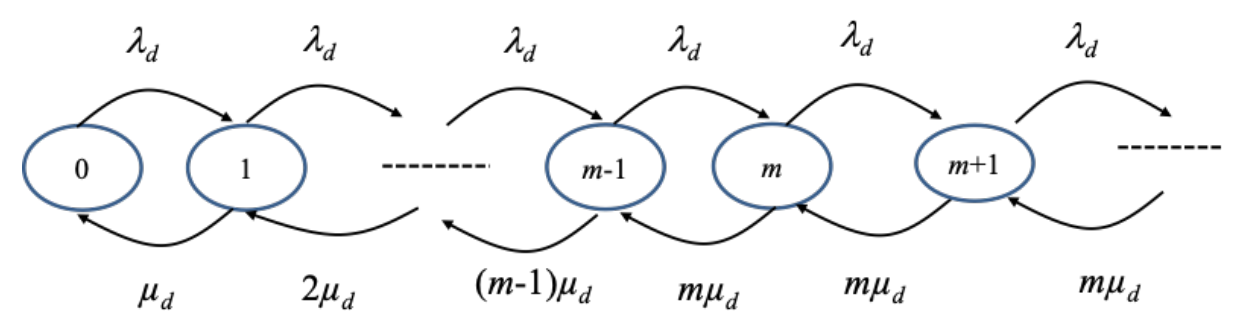

Figure 7. The $\mathrm{M} / \mathrm{G} / \mathrm{s} / \infty$ queueing model.

The $\mathrm{M} / \mathrm{M} / \mathrm{s} / \infty$ system is also referred to as the Erlang queueing model in Section 3.4.3 because it accommodates a queue when all superchargers are busy. The number of EVs in the supercharging queue is the sum of the number $N_{q}$ of vehicles waiting in the queue and the number $N_{c}$ undergoing DC charging. The expected values can be obtained, respectively, as follows:

$$
\begin{aligned}
& E\left[N_{q}\right]=\frac{\phi C(m, s)}{m-\phi}, \\
& E\left[N_{c}\right]=\frac{\lambda_{b} B(s)}{\mu_{d}} .
\end{aligned}
$$

For batteries, the difference between charging in the charging bay versus charging by supercharging is mainly the charging rate. A supercharger uses $480 \mathrm{~V}$ level-3 charging technology with a power of up to $80 \mathrm{~kW}$. Conversely, a charging bay used for recharging depleted swapped batteries uses level-2 charging technology, which uses medium voltage and power to preserve battery life.

\subsubsection{Electric power demand}

Let $P_{b}$ be the power used to recharge a depleted battery in the charging bay and $P_{d}$ be the power used to recharge a depleted battery using a supercharger. The total power $P_{s}$ used by a station is then the sum of the power used in the charging bay and that used by the superchargers:

$$
P_{s}=P_{b} E\left[N_{b}\right]+P_{d} E\left[N_{c}\right]=\frac{\lambda_{b}}{\mu_{b}} P_{b}+\lambda_{b} B(s)\left(\frac{P_{d}}{\mu_{d}}-\frac{P_{b}}{\mu_{b}}\right)=P_{b} \theta(1-B(s))+\phi P_{d} .
$$

Given $P_{b}$ and $P_{d}$, the average power used by a station depends on the arrival $\theta$ and the number $s$ of batteries in the charging bay but is independent of the 
number $m$ of superchargers. Because $\lambda_{b}$ is an exogenous variable, we present the following properties to characterize the relation between $s$ and $P_{s}$ :

Theorem 2. If $P_{d} \mu_{b}>P_{b} \mu_{d}$, then $P_{s}$ decreases monotonically with s. If $P_{d} \mu_{b}<P_{b} \mu_{d}$, then $P_{s}$ increases monotonically with $s$.

Proof. From the equation for $P_{s}$, if $P_{d} \mu_{b}<P_{b} \mu_{d}$, then $P_{d} \mu_{b}-P_{b} \mu_{d}<0$. According to Lemma $3, B(s)$ decreases monotonically with $s$, so $P_{s}$ increases monotonically with $s$. Similarly, if $P_{d} \mu_{b}>P_{b} \mu_{d}$, then $P_{d} \mu_{b}-P_{b} \mu_{d}>0$. Given that $B(s)$ decreases monotonically with $s, P_{s}$ must decrease monotonically with $s$.

Theorem 3. If $P_{d} \mu_{b}=P_{b} \mu_{d}$, i.e., the charging bay and the superchargers use the same charging technology, then the two-stage priority queuing network reduces to a single $M / G / s / \infty$ queue.

Proof. If the charging bay uses the same charge technology as the superchargers, the time to charge a battery is identical for both processes. When an arriving EV is blocked due to a shortage of spare batteries, it will prefer to wait for the next available spare battery instead of using superchargers because the expected waiting time in the swap queue will not exceed the time to complete the supercharging process. Since superchargers are no longer used, the service process is reduced to an $\mathrm{M} / \mathrm{G} / \mathrm{s} / \infty$ queue.

\subsection{The Mathematical Model}

This section presents two models for HCS services. The first model pre-defines as service target the probability of battery stockout and the probability of queueing for superchargers, whereas the second model pre-defines as service target the total sojourn time in stations.

4.5.1 Optimal location and inventory for hybrid charging station with service-level constraints

We now present a network design model in which all stations can exchange batteries and supercharge at the same time. The OLIHCS-SL model minimizes the 
total infrastructure cost subject to power capacity, probability of battery stockout, and queueing for supercharging:

$$
\begin{aligned}
& (\text { OLIHCS-SL }) \min \sum_{j=1}^{J}\left(F_{j} x_{j}+F_{b} s_{j}+F_{d} m_{j}\right) \text {, } \\
& \text { s.t. } \quad \sum_{j} y_{i j}=1 \quad \forall i, \\
& y_{i j} \leq d_{i j} x_{j} \quad \forall i, j, \\
& \theta_{j}=\lambda_{j} / \mu_{b} \quad \forall j, \\
& \lambda_{j}=\sum_{i} y_{i j} \lambda_{i} \quad \forall j, \\
& B_{j}\left(s_{j}\right) \leq \gamma \quad \forall j, \\
& B_{j}\left(s_{j}\right)=\frac{\theta_{j}^{s_{j}} / s_{j} !}{\sum_{k=0}^{s_{j}}\left(\theta_{j}^{k} / k !\right)} \quad \forall j \\
& \phi_{j}=B_{j}\left(s_{j}\right) \lambda_{j} / \mu_{d} \quad \forall j, \\
& C_{j}\left(m_{j}\right) \leq \beta \quad \forall j, \\
& \frac{\frac{\phi_{j}^{m}}{m !\left(1-\phi_{j} / m\right)}}{\sum_{k=0}^{m-1} \frac{\phi_{j}^{k}}{k !}+\frac{\phi_{j}^{m}}{m !\left(1-\phi_{j} / m\right)}}=C_{j}\left(m_{j}\right) \quad \forall j, \\
& P_{b} \theta_{j}\left(1-B_{j}\left(s_{j}\right)\right)+\phi_{j} P_{d} \leq P_{j} \quad \forall j, \\
& s_{j} \leq M x_{j} \quad \forall j, \\
& s_{j} \in \mathbb{Z}^{+} \quad \forall j, \\
& m_{j} \in \mathbb{Z}^{+} \quad \forall j, \\
& x_{j} \in\{0,1\} \quad \forall j, \\
& y_{i j} \in\{0,1\} \quad \forall j \text {. }
\end{aligned}
$$

In the OLIHCS-SL model, the objective function (25) is comprised of station setup, battery, and supercharger-procurement costs. In addition to $x_{j}$ and $s_{j}$, the new decision variable $m_{j}$ is the number of superchargers in station $j$. Constraint (26) states that each customer zone must be covered by one station. Constraint (27) sets the maximum allowed distance from a customer zone to an assigned BSS. 
Constraint (28) calculates the expected EV arrivals at station $j$ during a battery recharge cycle in the charging bay. Constraint (29) aggregates the EV arrival rate at a BSS over all assigned TAZs. Constraint (30) defines the service-level requirement (i.e., battery stockout probability) and ensures that it does not exceed a pre-specified level (i.e., $\gamma=0.05$ ). Constraint (31) is the Erlang loss formula for calculating the stockout probability. Constraint (32) calculates the average number of vehicles directed to the supercharging bay due to a shortage of fully charged batteries. Constraint (33) states that the probability of an EV waiting for a supercharger must not exceed a pre-specified threshold (e.g., $\beta=0.05$ ). Constraint (34) is the Erlang queueing formula to calculate the probability of waiting. Constraint (35) states that the power consumed at any station should not exceed the grid capacity. Constraint (36) ensures that no battery is placed at a station unless the station is open. Finally, constraints (37)-(40) specify the decision-variable types for $s_{j}, x_{j}, m_{j}$, and $y_{i j}$, respectively.

4.5.2 Optimal location and inventory for hybrid charging station with service time constraints

The OLIHCS-ST model is similar to the OLIHCS-SL model except that it replaces the probability-based service-level constraint with the total sojourn-time 
constraint, so that the latter does not exceed a pre-defined threshold value:

$$
\begin{aligned}
& \text { (OLIHCS-ST) } \min \sum_{j=1}^{J}\left(F_{j} x_{j}+F_{b} s_{j}+F_{d} m_{j}\right) \text {, } \\
& \text { s.t. } \quad \sum_{j} y_{i j}=1 \quad \forall i, \\
& y_{i j} \leq d_{i j} x_{j} \quad \forall i, j, \\
& \theta_{j}=\lambda_{j} / \mu_{b} \quad \forall j, \\
& \lambda_{j}=\sum_{i} y_{i j} \lambda_{i} \quad \forall j, \\
& B_{j}\left(s_{j}\right)=\frac{\theta_{j}^{s_{j}} / s_{j} !}{\sum_{k=0}^{s_{j}}\left(\theta_{j}^{k} / k !\right)} \quad \forall j, \\
& \phi_{j}=B_{j}\left(s_{j}\right) \lambda_{j} / \mu_{d} \quad \forall j, \\
& \frac{\frac{\phi_{j}^{m}}{m !\left(1-\phi_{j} / m\right)}}{\sum_{k=0}^{m-1} \frac{\phi_{j}^{k}}{k !}+\frac{\phi_{j}^{m}}{m !\left(1-\phi_{j} / m\right)}}=C_{j}\left(m_{j}\right) \quad \forall j, \\
& W_{\max } \geq \frac{C_{j}\left(m_{j}\right) B_{j}\left(s_{j}\right)}{\mu_{d}\left(m_{j}-\phi_{j}\right)}+\frac{B_{j}\left(s_{j}\right)}{\mu_{d}}+\left(1-B_{j}\right) \tau \quad \forall j, \\
& P_{b} \theta_{j}\left(1-B_{j}\left(s_{j}\right)\right)+\phi_{j} P_{d} \leq P_{j} \quad \forall j, \\
& s_{j} \leq M x_{j} \quad \forall j, \\
& s_{j} \in \mathbb{Z}^{+} \quad \forall j, \\
& m_{j} \in \mathbb{Z}^{+} \quad \forall j, \\
& x_{j} \in\{0,1\} \quad \forall j, \\
& y_{i j} \in\{0,1\} \quad \forall j \text {. }
\end{aligned}
$$

In the OLIHCS-ST model, constraint (49) defines the total sojourn-time requirement and ensures that it does not exceed a pre-specified level. All other constraints and the objective function are the same as for the OLIHCS-SL model. 


\subsection{Solution Approaches}

The Erlang function is computationally challenging because the decision variables $s_{j}$ and $m_{j}$ are embedded in power and factorial functions. In fact, many off-the-shelf solvers generally cannot compute values for such highly nonlinear functions, let alone provide a global optimal solution for the optimization problem. Therefore, we develop a heuristic method based on the lemmas in Sections 3.4.2 for fast solutions.

\subsubsection{The metaheuristic solution method}

The heuristic algorithm is based on the optimal customer allocation policy from Section 3.6.1. Its goal is to determine which candidate location should be chosen to open a station.

The objective function in the model has only three items: setup cost, battery procurement cost, and supercharger-procurement cost. We design a metaheuristic algorithm containing subroutine algorithms (7) through (10) to solve for a quality solution. Figure ?? shows the flow chart of the overall metaheuristic.

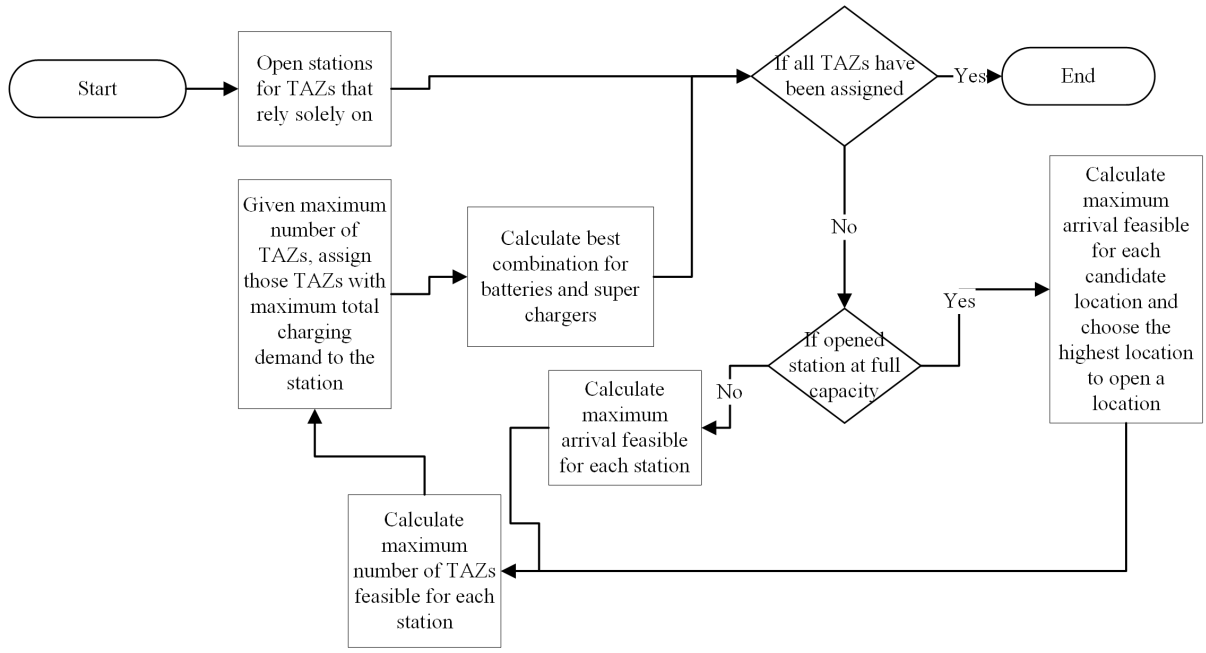

Figure 8. The flow chart of the metaheuristic for the OLIHCS-SL and the OLICHS-ST models 
4.6.2 A metaheuristic algorithm for the OLIHCS-SL and the OLIHCS-ST models

To maintain the desired service target, an important step in the HCS location-allocation decision is to convert power capacity to the number of EVs that a station can handle. For a given service-level threshold and $P_{j}$ at station $j$, Algorithm 7 gives the process of converting the power capacity to deal with the maximum allowable EV arrival rate for the models. Based on Lemmas 3 and 6, the algorithm increases the number of spare batteries and the number of superchargers until it meets the desired service level. The process repeats by increasing $\lambda_{j}$ until the power capacity of station $j$ is violated.

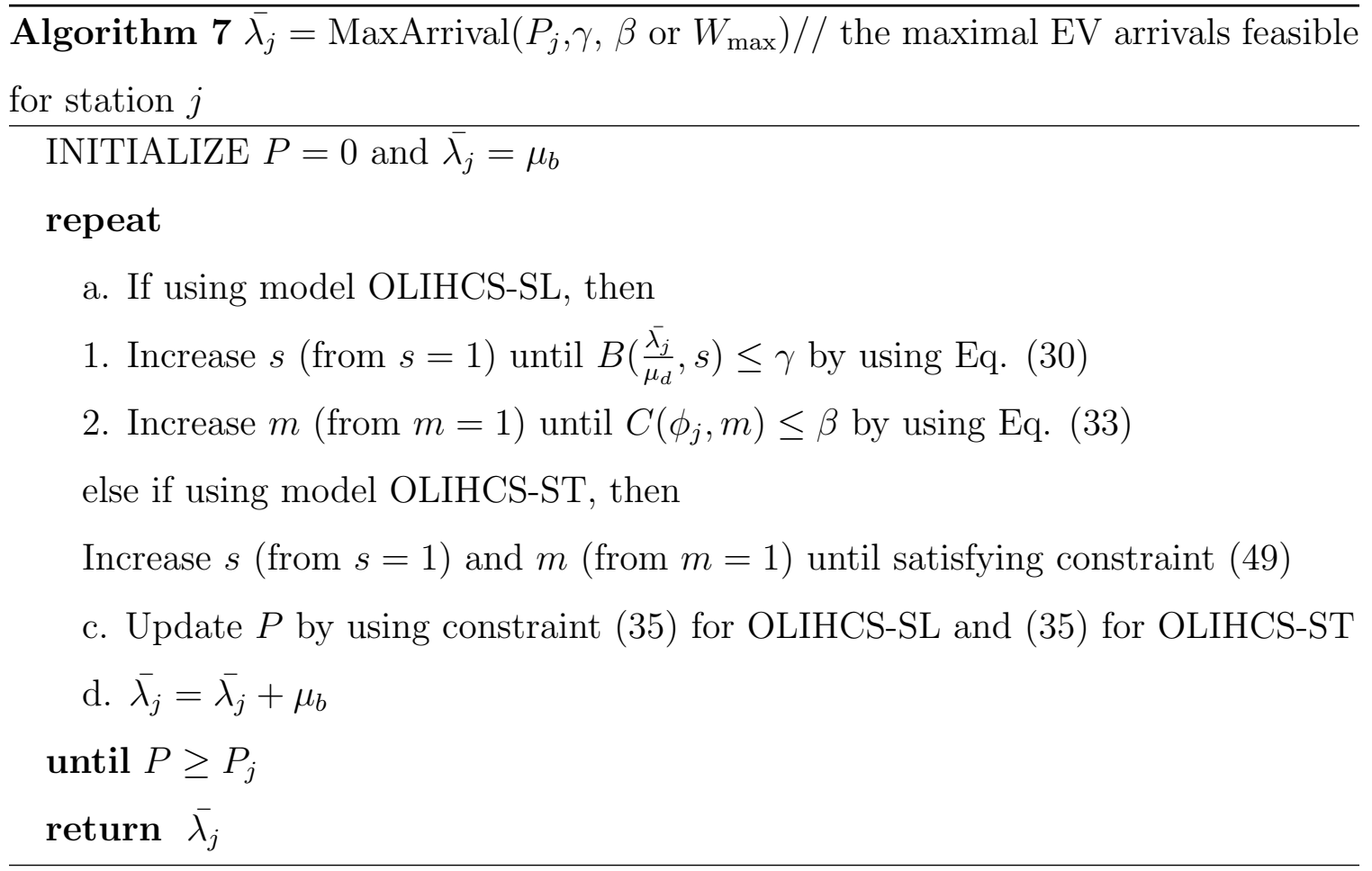

By using the maximal arrive rate of each candidate station $\overline{\lambda_{j}}$ determined by Algorithm 7, we compute the maximum number $N_{j}$ of TAZs that can be assigned to station $j$. This is because constraints (26) and (42) require that all TAZs be covered. To minimize total setup cost, the optimal solution opts to open the fewest possible stations, with each station serving as many TAZs as possible. Algorithm 8 below first sorts all TAZs that can be covered by station $j$ in ascending order of their EV arrival rates $\lambda_{j}$. It then chooses the first $N_{j}$ TAZs in the sorted list until 
the maximum arrival rate $\overline{\lambda_{j}}$ is reached; thus $N_{j}$ is returned as the maximum number of zones that station $j$ can cover. Note that we use a sorted list to determine $N_{j}$, not the final assignment of ( $N_{j}$ or fewer) TAZs to station $j$. The latter is determined by Algorithm 8.

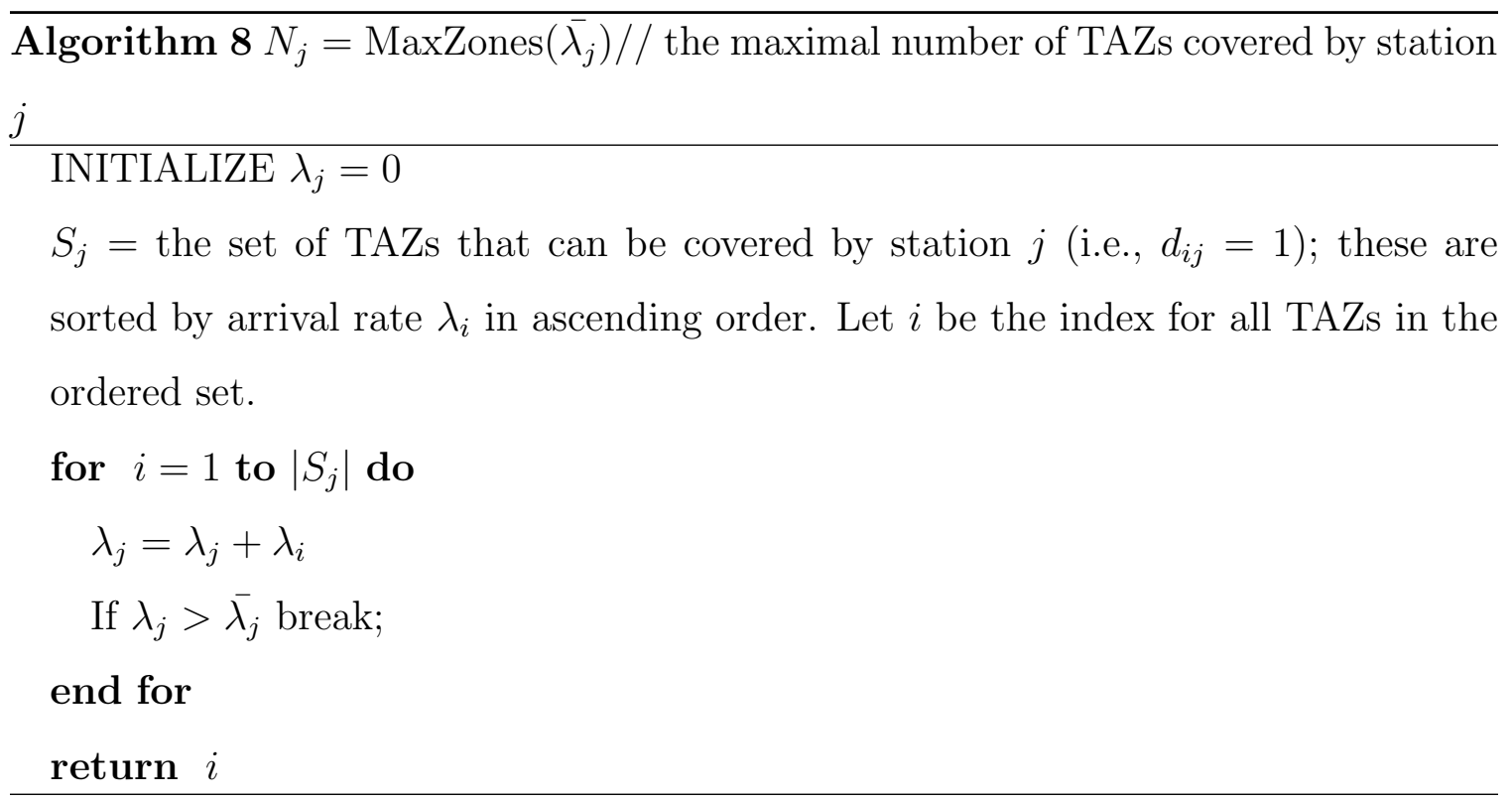

By using the maximum number of TAZs that station $j$ can cover, Algorithm 9 actually allocates the TAZs to the station by enumerating all combinations of $N_{j}$ TAZs and choosing the combination that yields the maximum arrival rate for the station.

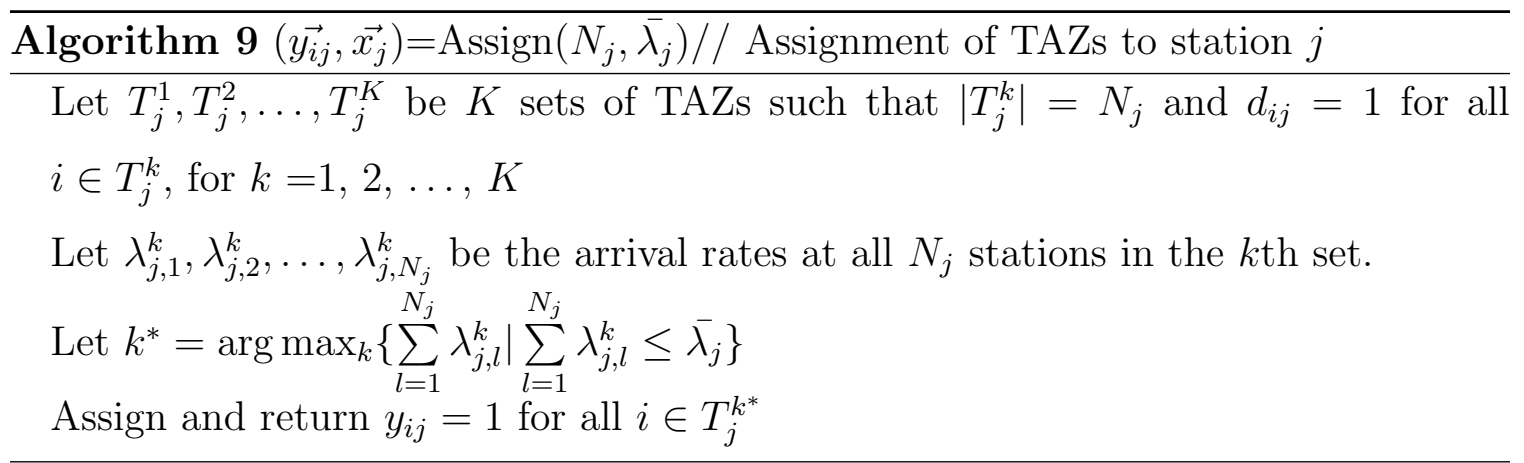

Once the assignment of TAZs to candidate station $j$ is completed, the allocation of batteries and superchargers are obtained by Algorithm 10. As mentioned previously, to minimize the objective function, the least number of 
batteries and superchargers are preferred so long as the service-level constraints are met. On the other hand, since increasing the number of spare batteries reduces the required superchargers, it is perhaps advantageous to overachieve the service level $\gamma$ in an effort to reduce $m$ to 1 , provided the power constraint is not violated since the cost of installing a supercharger is greater than that of purchasing a spare battery.

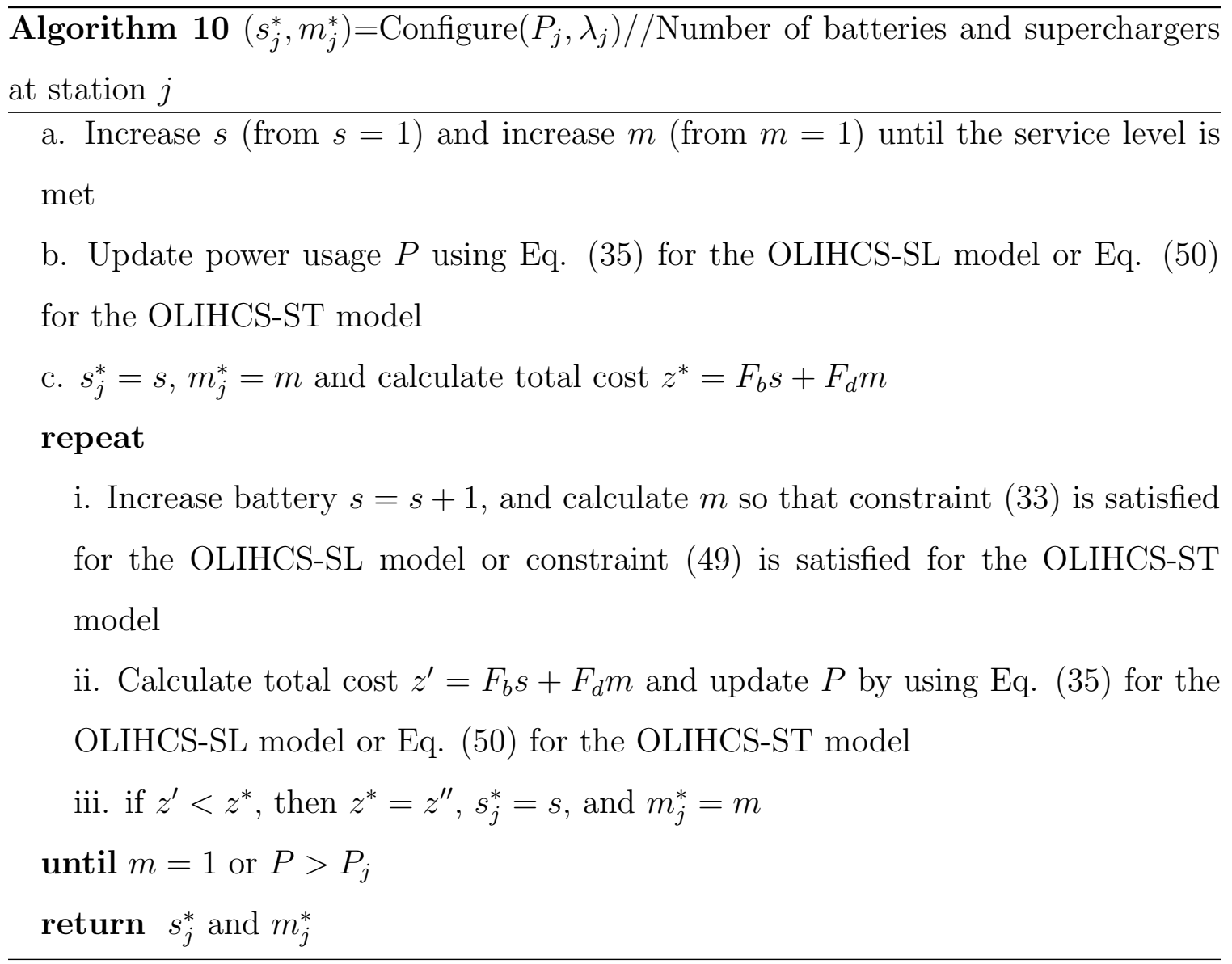

Algorithm 11 below integrates Algorithms 7-10 to provide a heuristic solution to the models. Note that the solution can be obtained by simply removing the procedures related to determining the optimal number of superchargers from Algorithm 11. In particular, Algorithm 11 first uses Algorithms 7 and 8 to calculate the maximum number of TAZs that a candidate station is capable of covering, then uses Algorithm 9 to assign TAZs to all opened stations, and finally determines the numbers of spare batteries and superchargers for each station. 


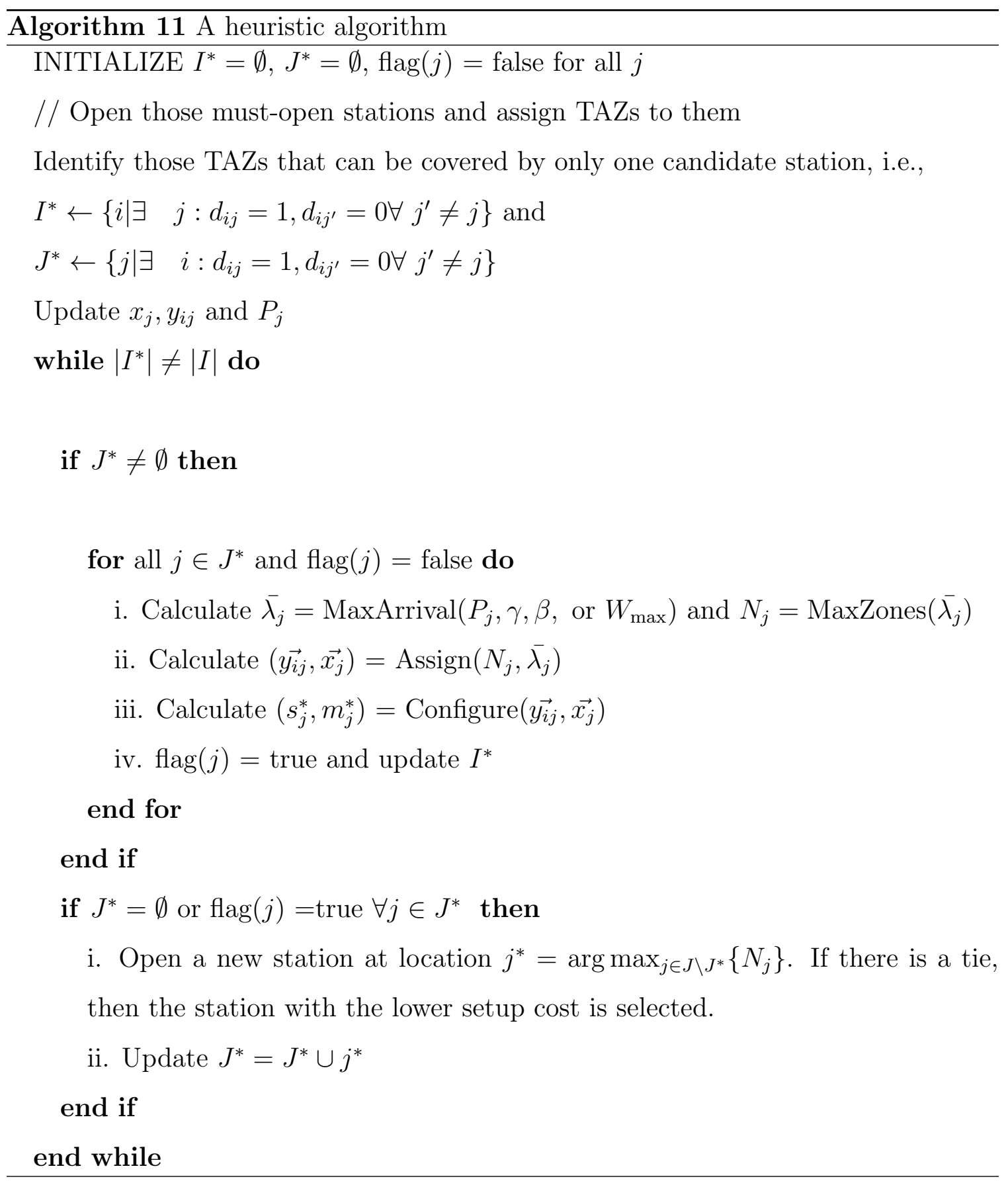

Since the Erlang loss constraint and the Erlang queuing constraint are nonlinear, we use Tabu search to further improve the solution from Algorithm 11. In particular, the Tabu search in Algorithm 12 reassigns TAZs to different stations with the promise of potentially closing some stations, thus minimizing the total cost. The Tabu search, first created by Glover [81], is a metaheuristic search method 
that finds the best solution in a defined neighborhood at every iteration to escape from local optima.

A Tabu list is used to track all historical solutions for higher efficiency. In particular, we consider the following two types of neighborhood functions:

Substitution: Randomly select two unopened candidate stations. If the total setup cost of the two stations is less than that of an opened station, then open these two stations and re-assign the TAZs to them and to other opened stations. This neighborhood function is motivated by the fact that Algorithm 5 gives the priority to stations based on the' maximum number $N_{j}$ of zones, not on the setup cost.

Consolidation: Randomly select a number of TAZs covered by a currently opened station $j$. Move the TAZs to another opened station provided the power capacity is not violated. This neighborhood function can reduce the number of batteries required in station $j$ and possibly the total cost.

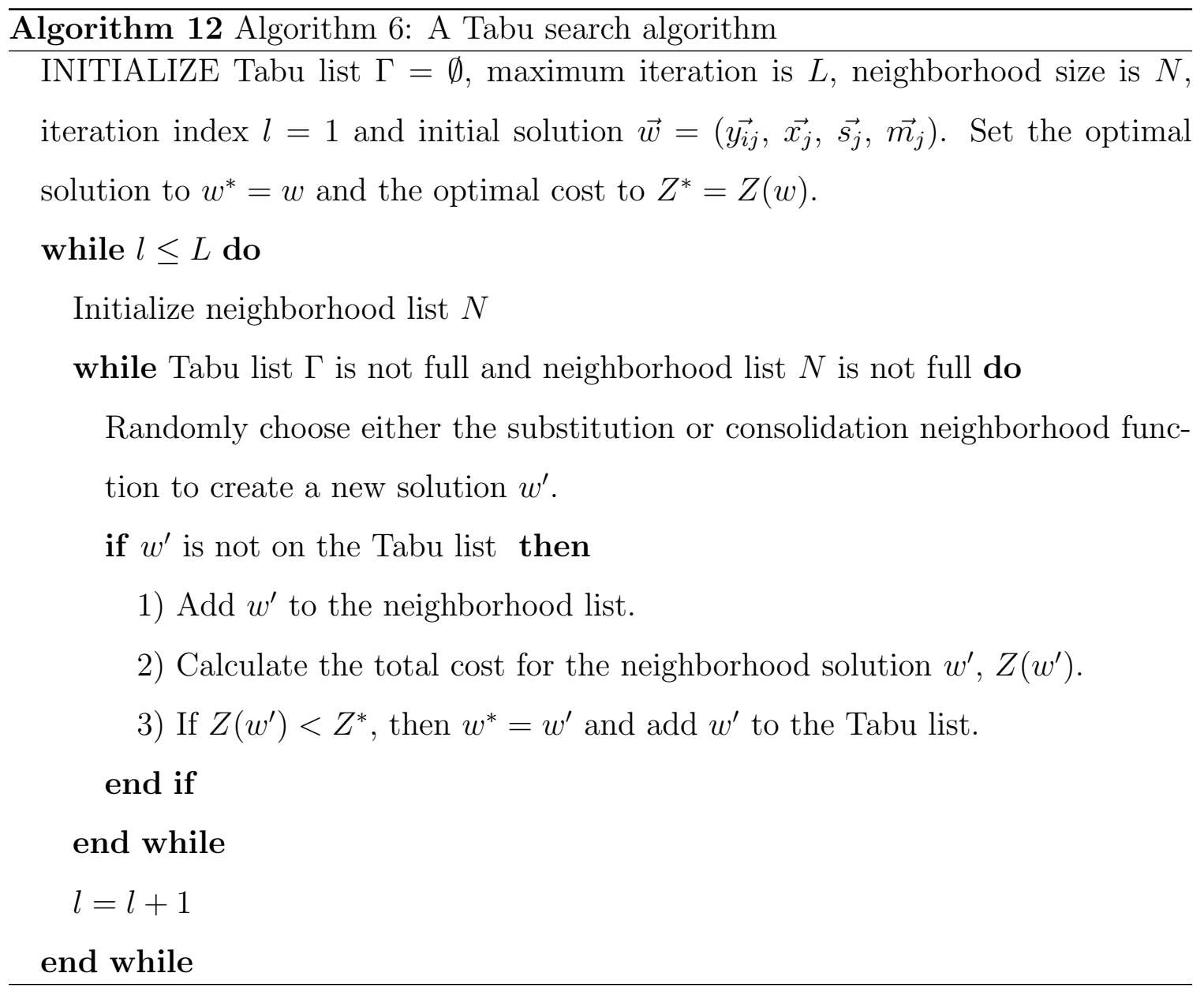


4.7 An illustrative example for the metaheuristic method

We illustrate the implementation of algorithms for the OLIHCS-SL model by using an example with six TAZs and three candidate-station locations. The data in Table 10 include information on $d_{i j}$, the power capacity and setup cost for each candidate station, and EV arrival rate in each TAZ. We set $\gamma=0.2$ and $\beta=0.2$ in the numerical experiment. We first use Algorithm 7 to calculate the maximum EV arrivals that each candidate location can potentially serve. As a result, $\overline{\lambda_{1}}=17.75$, $\bar{\lambda}_{2}=16.75$, and $\bar{\lambda}_{4}=20.5$. Next, we apply Algorithms 8 and 9 to calculate the maximum number of TAZs covered by each station and furthermore find that Station 1 covers TAZs 3, 4, and 6; Station 2 covers TAZs 4 and 6; and Station 3 covers TAZs 3, 6, and 1. Since Stations 1 and 3 can serve three TAZs whereas Station 1 has a lower setup cost, we opt to open Station 1 first. Subsequently, we assign TAZs 1, 4, and 6 to Station 1. Next, we need to cover TAZs 2, 3, and 5. Realizing Station 2 is only able to cover TAZs 1 and 2 whereas Station 3 can cover TAZs 2, 3, and 5, we open Station 3 as the second service facility. After the assignment is completed, we further determine the optimal quantity of batteries and superchargers. Station 1 is expected to serve $15 \mathrm{EVs}$ per hour, so by Lemmas 1 and $4, s_{1}=52$ batteries and $m_{1}=4$ superchargers with the total cost of $\$ 544000$ and total power of $585.78 \mathrm{~kW}$, which corresponds to $\gamma=0.19$ and $\beta=0.07$. We then continue increasing $s_{1}$ until either the power exceeds the given capacity or $m_{1}$ drops to 1 . Finally, among all potential configurations, $s_{1}=53$ and $m_{1}=3$ yield the lowest cost of $\$ 506000$ with a power demand of $586.74 \mathrm{~kW}$ (below the capacity). This solution yields the actual service levels of $\gamma=0.18$ and $\beta=0.18$. The same process is repeated for Station 3. After applying the Tabu search we obtain the final solution, where Station 1 has 53 batteries and three superchargers, and Station 3 has 57 batteries and three superchargers, and the total network cost is $\$ 1.79$ million.

TABLE 10. Data for TAZs and candidate-station locations. 


\begin{tabular}{ccccc}
\hline TAZ $\backslash$ Station & $j=1$ & $j=2$ & $j=3$ & $\lambda_{i}(\mathrm{EV} /$ hour $)$ \\
\hline$i=1$ & 1 & 0 & 1 & 6 \\
$i=2$ & 0 & 1 & 1 & 8 \\
$i=3$ & 1 & 0 & 1 & 3 \\
$i=4$ & 1 & 1 & 0 & 4 \\
$i=5$ & 0 & 1 & 1 & 9 \\
$i=6$ & 1 & 1 & 1 & 5 \\
\hline Power capacity $P_{j}(\mathrm{~kW})$ & 700 & 650 & 800 & \\
Setup cost $F_{j}(\times \$ 1,000)$ & 300 & 500 & 450 & \\
\hline
\end{tabular}




\section{CHAPTER 5}

\section{COMPUTATIONAL RESULTS}

In this section, we discuss results from our numerical experiments for the four models proposed in Chapters 3 and 4 .

\subsection{EV Charging-Demand Forecasting}

We consider the EV charging demand in an area of interest. Each area is divided into small TAZs. We used an expanded network construction to estimate the EV charging demand in each TAZ. Each EV trip that can be served by a sequence of BSSs can be represented by a path on the expanded network. The path is obtained by solving a shortest-path problem whose formulation contains constraints that ensure that the trips can be accomplished without exhausting the battery. In this study, instead of completely exhausting a battery, an EV goes to a station before its battery reaches a minimum acceptable level. The distance covered by an EV between a given initial SOC and when its battery reaches a minimum acceptable level is defined as the allowable driving range.

In the original network, each node stands for a candidate location for setting up BSSs. Figure 9 illustrates an example with a single path $q$ whose visited nodes are $N^{q}=\{A, B, C, D\}$. The arcs between nodes connect these nodes together. The set of arcs on path $q$ is $A^{q}=\{(A, B),(B, C),(C, D)\}$. Each trip of an EV has an original and final TAZ, and multiple intermediate TAZs through which it passes. The extended network approach is described as follows: 


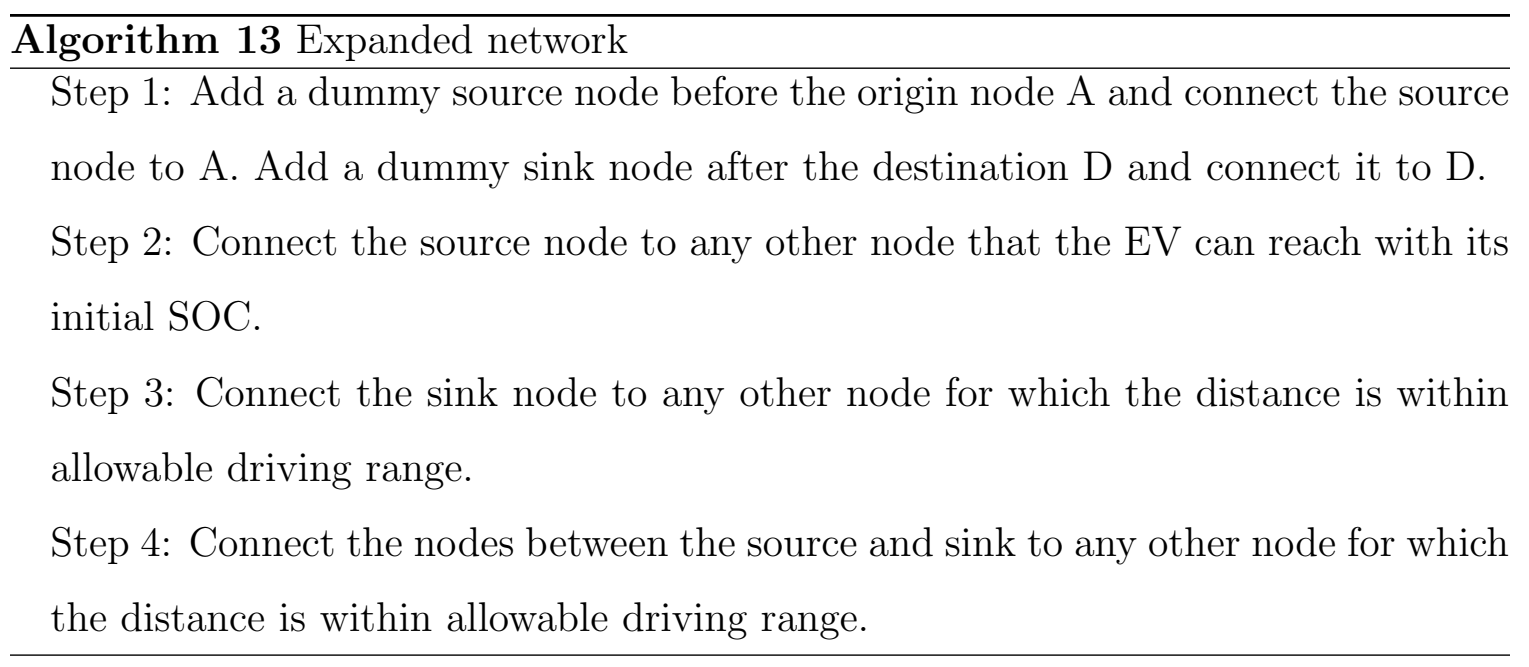

In the expanded network, each arc indicates a path with a battery-swap service. Each arc combination indicates that an EV can finish the trip without exhausting the battery.

First, each EV chooses the shortest distance possible to finish the trip. Second, a linear relationship exists between power consumption and driving distance. Third, all traveling EVs must gain access to a charging station before their battery reaches a minimum acceptable level (e.g., 30\% SOC).

EV charging demand in a single TAZ is defined as the number of EVs in the given TAZ with batteries at the minimum acceptable charge level (so they need to access a charging station). To illustrate the trip model, we consider an EV with a full battery range of 100 miles. Suppose that the EV has a trip that passes through four TAZs. Assuming that power consumption is linear in travel distance, the EV loses 1\% SOC per mile and depletes its battery when it travels 100 miles. An EV must find a station when the SOC drops to $25 \%$ (i.e., the remaining battery range is 25 miles) and its initial SOC is 50\%. It then needs to charge at node A before passing node $\mathrm{B}$, because we cannot deplete the battery when it arrives in the station. After charging at node A, it will have a sufficient SOC to travel to node C and arrive at its destination node D. The result of such a trip is shown in Figure 9. 


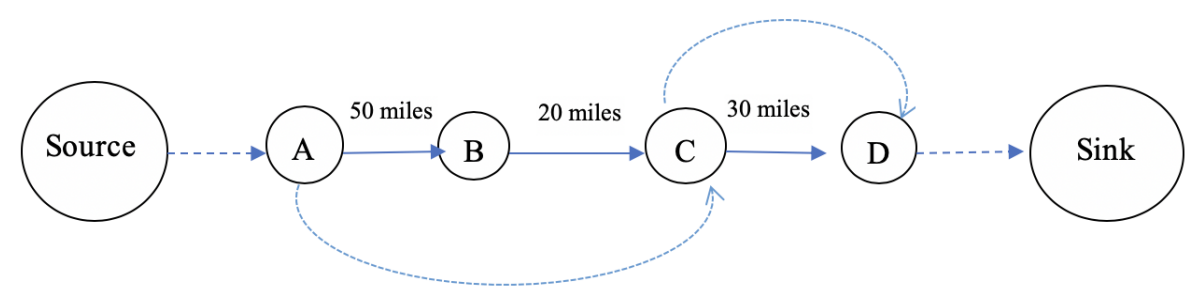

Figure 9. Four-node trip by an EV.

The indices for the shortest path in the expanded network is given below.

\begin{aligned} & TABLE 11. Sets and indices \\ & \hline$j \in J, i \in I$ Set of TAZs \\ &$q \in Q$ Set of EV trips \\ & \hline\end{aligned}

TABLE 12. Parameters and indices.

$D_{i j} \quad$ Distance between TAZ $i$ to $j$

$M_{q} \quad$ TAZ in which trip $q$ starts

$N_{q} \quad$ TAZ in which trip $q$ ends

$C R$ Distance an EV can travel after charging service

$S_{q} \quad$ Distance an EV can travel with its initial SOC

TABLE 13. Decision variables.

$x_{i, j}^{q} \quad$ Binary variable equals to 1 if trip $q$ travels from TAZ $i$ and is charged at TAZ $j$ or 0 otherwise

$d_{j} \quad$ EV charging demand at TAZ $j$ 


$$
\begin{aligned}
\min Z & =\sum_{i, j, q}\left(x_{i, j}^{q} \times D_{i j}\right) \\
\text { s.t. } \sum_{j} x_{i, j}^{q}-\sum_{j} x_{j, i}^{q} & =\left\{\begin{array}{r}
1 \quad i=M_{q} \\
-1 \quad i=N_{q} \quad \forall q, i \\
0 \quad i \neq M_{q}, N_{q},
\end{array}\right. \\
\sum_{j} x_{i, j}^{q} D_{i, j} \leq S_{q} \quad i=M_{q} \quad \forall q, & \forall x_{j} x_{i, j}^{q} D_{i, j} \leq C R \quad i \neq M_{q} \quad \forall q, i, \\
\sum_{i, q} x_{i, j}^{q} & =d_{j} \quad j \neq M_{q} \quad \forall i, j .
\end{aligned}
$$

The objective (56) is to minimize the distance and the number of stops each trip has to make. Constraint (57) is the flow-balance constraint. Constraint (58) states that, when an EV starts a trip, it has to ensure that the trip will not deplete its initial SOC. Constraint (59) states that, as an EV passes through intermediate TAZs, it must look for a station at a total distance less than CR, which is a pre-defined parameter that gives the allowable range. Constraint (60) calculates the EV charging demand of each TAZ.

TABLE 14. An example distance table.

\begin{tabular}{cccc} 
Distance & 1 & 2 & 3 \\
\hline 1 & 0 & 96 & 67 \\
2 & 96 & 0 & 57 \\
3 & 67 & 57 & 0 \\
\hline
\end{tabular}

For example, Table 14 is the distance table for an example with five trips and three TAZs. Let the range of the $\mathrm{EV}$ be $\mathrm{CR}=120$ miles, the initial SOC of each trip $S_{q} \sim U(60,120), M_{q} \neq N_{q} \sim U(1,3)$. We use CPLEX to solve the model. The result is shown in Table 15.

TABLE 15. The result of EV charging-demand forecasting example. 
Trip No. Start range Start TAZ End TAZ Distance to travel Intermediate TAZ

\begin{tabular}{lccccc}
\hline 1 & 114 & 3 & 1 & 67 & N/A \\
2 & 97 & 3 & 2 & 57 & N/A \\
3 & 60 & 2 & 3 & 57 & N/A \\
4 & 94 & 2 & 1 & 96 & 2 \\
5 & 81 & 1 & 2 & 96 & 1 \\
\hline
\end{tabular}

In this example, Trips 1-3 have sufficient battery charge to finish the trips.

However, Trips 4 and 5 have to swap batteries or obtain a charging service to finish the trips. Therefore, TAZs 4 and 5 each have one EV charging demand in this case.

TABLE 16. Charging topologies.

\begin{tabular}{|c|c|c|c|}
\hline Level & Location & Voltage and current & Approx. time to charge \\
\hline 1 & Residential & $110 \mathrm{~V}, 15 \mathrm{~A}$ & 18 Hours \\
\hline 2 & Residential/public & $220 \mathrm{~V}, 15-30 \mathrm{~A}$ & $3-6$ Hours \\
\hline 3 & Commercial & $480 \mathrm{~V}, 167 \mathrm{~A}$ & $20-50$ minutes \\
\hline
\end{tabular}

\subsection{Settings of Other Parameters in the Four Models}

To calculate the stockout and queueing probability by using the Erlang loss and queueing functions, we need the average service or charging times. Table 16 displays three charging levels and their charging times. In our experiment, we assume that the empty batteries taken out of the EV have less than a level-2 charge because type 1 is too slow and is commonly used for residential, whereas type 3 requires more investment in infrastructure and has a smaller EV market share.

For each experiment associated with each of the four models, various operational conditions are considered, such as EV arrival rate, service level, and the probability of waiting for superchargers. We test the algorithm for the five different design sets given in Table 17. In each experiment, a total of 50 instances with ten for each set are randomly generated from the EV charging-demand generation model, as described in Section 5.1. Each set has its distinct network size of locations 
and TAZs. For instance, Set 5 has 200 candidate-station locations and 1000 TAZs. Note that the ratio is defined as the number of TAZs divided by the number of candidate locations. The proposed algorithm was coded in Python with the Numpy plug-in. All tests were run on an Acer V3-372T with a $2.3 \mathrm{GHz}$ dual-core I5 processor and $8 \mathrm{~GB}$ of RAM.

\begin{tabular}{cccc}
\multicolumn{4}{c}{ TABLE 17.} \\
\hline Set & Number of locations & Number of TAZs & Ratio \\
\hline 1 & 5 & 10 & 2 \\
2 & 10 & 20 & 2 \\
3 & 20 & 50 & 2.5 \\
4 & 50 & 200 & 4 \\
5 & 200 & 1000 & 5 \\
\hline
\end{tabular}

A Nissan Leaf with a $24 \mathrm{kWh}$ battery is the object of our experiment. Its average charging time is $\tau_{c}=4$ hours for the swapped batteries, and $\tau_{g}=0.5$ hour if using a supercharger. The corresponding charge rates are $\mu_{b}=1 / \tau_{c}=0.25$ batteries/hour and $\mu_{d}=1 / \tau_{g}=2 \mathrm{EV} /$ hour, respectively. Other parameters in the models are $F_{j} \sim U(\$ 200000, \$ 500000), F_{b}=\$ 7,000, F_{d}=\$ 45,000, P_{j} \sim U(600 \mathrm{~kW}$, $800 \mathrm{~kW}), \lambda_{i} \sim U(1,2)$ in units of $\mathrm{EV} /$ hour, and $d_{i j} \in\{1,0\}$. battery swapping time is set to 6 minutes. Furthermore, for the OLIBSS-SL model, $\gamma \in\{0.01,0.05,0.1$, $0.15,0.2\}$ is tested. For the OLIHCS-SL model, $\gamma, \beta \in\{0.05,0.1,0.15,0.2\}$, a total of 16 scenarios is tested. For the OLIBSS-ST model and the OLIHCS-ST model, $W_{\max } \in\{10$ min., 15 min., 20 min., 25 min., 30 min. $\}$ is tested.

\subsection{Numerical Results for the OLIBSS-SL Model}

Table 18 shows the average results for 50 randomly generated instances in five sets for the OLIBSS-SL model when $\gamma=0.2$. The number in each cell is the average result over 10 instances in each set. When the network size increases, the numbers of required charging stations and total number of batteries both increase. The average number of batteries per station increases from 21.8 to 77.4. Second, when 
the network size increases, the average number of batteries per station increases as well. But when the size increases to the level as in Set 3 and above, the average number of batteries per station stabilizes around 75 because the power constraint becomes a binding constraint that prevents the station from serving more EVs.

TABLE 18. Average results of the OLIBSS-SL model with $\gamma=0.2$.

\begin{tabular}{ccccc}
\hline Set & Network cost $\left(\times \$ 10^{6}\right)$ & Average \# of BSS & Average \# of batteries & Average \# of batteries per station \\
\hline 1 & 1.2668 & 2.5 & 52.6 & 21.8 \\
2 & 1.6009 & 2.9 & 98.6 & 35.1 \\
3 & 3.8955 & 5 & 368.8 & 73.8 \\
4 & 9.5916 & 12.3 & 931.7 & 75.8 \\
5 & 47.292 & 60 & 4645.6 & 77.4 \\
\hline
\end{tabular}

TABLE 19. Total cost $(\times \$ 1,000)$ and number of stations with various $\gamma$.

\begin{tabular}{cccccc}
\hline Set & $\gamma=0.01$ & $\gamma=0.05$ & $\gamma=0.1$ & $\gamma=0.15$ & $\gamma=0.2$ \\
\hline 1 & $1465.6(2.5)$ & $1385.8(2.5)$ & $1337.5(2.5)$ & $1299.7(2.5)$ & $1266.8(2.5)$ \\
2 & $1936.2(2.9)$ & $1805.3(2.9)$ & $1720.6(2.9)$ & $1656.2(2.9)$ & $1600.9(2.9)$ \\
3 & $5299.7(6.1)$ & $4845.6(6)$ & $4524.9(5.8)$ & $4173(5.2)$ & $3895.5(5)$ \\
4 & $13359.1(15.5)$ & $12097.7(14.4)$ & $11136.8(13.8)$ & $10346.2(13.1)$ & $9591.6(12.3)$ \\
5 & $66007.2(75.6)$ & $59800.4(72)$ & $54822.4(68.4)$ & $50937.6(64)$ & $47292(60)$ \\
\hline
\end{tabular}

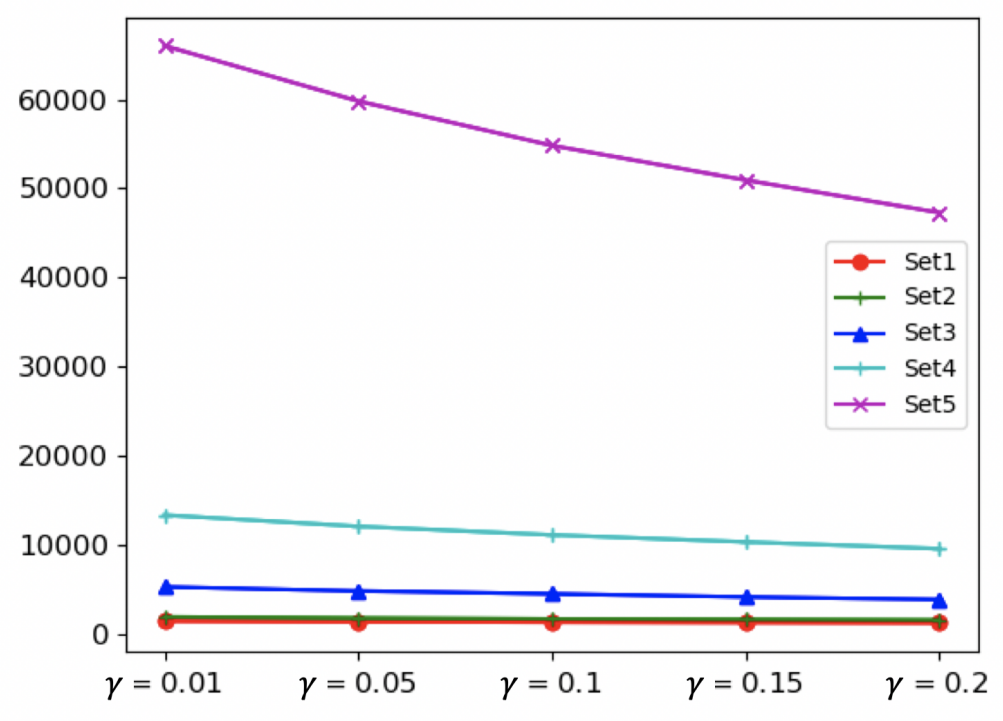


Figure 10. Total network-infrastructure cost as a function of $\gamma$.

Table 19 shows the cost and number of stations (in parentheses) with service levels incremented by $5 \%$. Figure 10 shows the network total cost as a function of $\gamma$. The results show that higher service levels require higher total network-infrastructure cost. Two elements contribute to the higher cost: First, higher service level requires more battery inventory for stations to guarantee that incoming EVs have a good chance to obtain a fully charged battery for swapping. Second, higher service levels require swapping stations to increase power consumption but, since the power capacity of stations is fixed, more battery swapping stations will have to open to serve all the EVs in the network, which in turn will increase the total network facility setup cost.

Figure 11 shows the percent total cost increase as a function of $\gamma$. The results suggest that, for small network size (i.e., Sets 1 and 2), total network cost increases steadily with service level. For example, for Set 1 , the total cost increases by $2.6 \%$ from $\gamma=0.2$ to 0.15 and $2.91 \%$ from 0.15 to 0.1 . But when network size is medium to large (i.e., Sets 3-5), the total cost increment does not follow a specific pattern since the setup cost starts to have an impact. The total cost of Set 3 increases by $8.43 \%$ from $\gamma=0.15$ to 0.1 but increases only by $7.09 \%$ from $\gamma=0.1$ to 0.05 . Second, Sets 1 and 2 have an average of 2.5 and 2.9 stations to open each case across all $\gamma$. Sets $3-5$ have more stations to open when the service level increases. For instance, Set 3 contains only five stations when $\gamma=0.2$ but the number increases to 5.2 and 5.8 for $\gamma=0.15$ and 0.1 , respectively. The increase occurs because the power capacity of a single station prevents it from serving more EVs in its area. Therefore, more stations are needed to serve the other EVs in the network. 


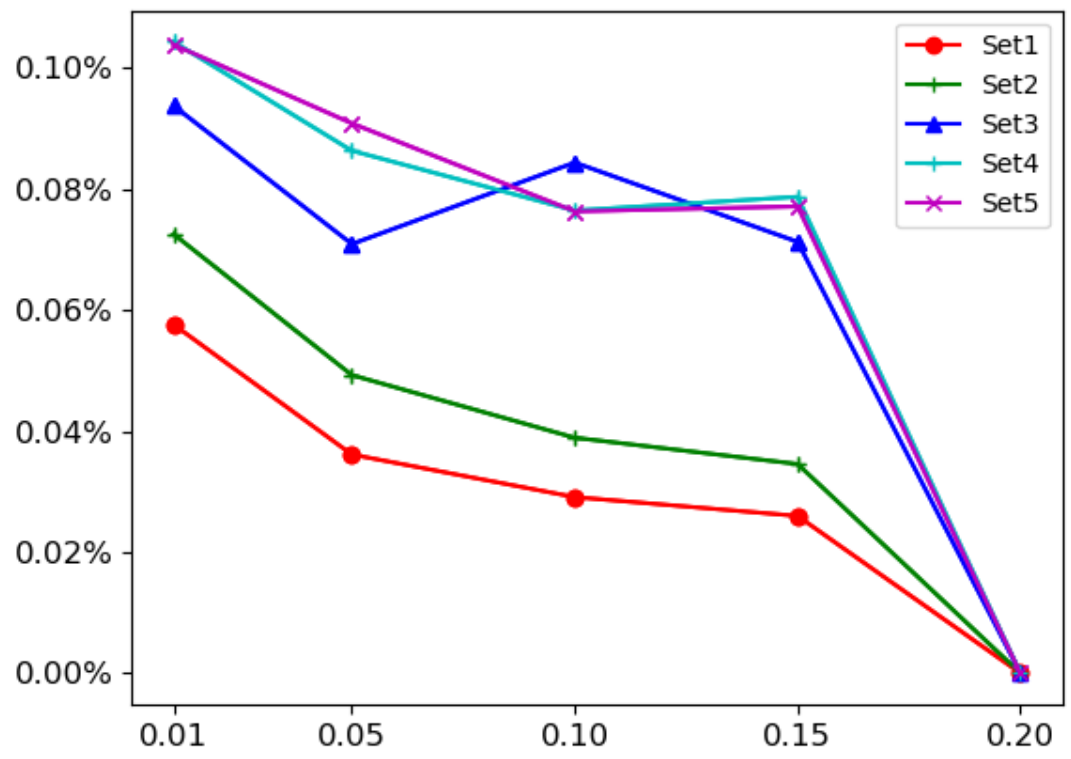

Figure 11. Percent change in total network infrastructure cost as a function of $\gamma$.

\subsection{Numerical Results for the OLIBSS-ST Model}

The same 50 instances with 10 for each set are used to test the OLIBSS-ST model. For $W_{\max }=10$ minutes, Table 20 summarizes the results for the infrastructure cost, the number of charging stations, and the battery quantity for five sets. The decimal values result from averaging ten instances in each set. As expected, the number of BSSs required and the battery inventory both increase as the network size increases. From Sets 1 to 5, the average number of batteries per station increases from 30.91 to 83.21 , respectively.

\begin{tabular}{ccccc}
\multicolumn{5}{c}{ TABLE 20. Average results for the OLIBSS-ST model for $W_{\max }=10$ minutes. } \\
\hline Set & Network cost $\left(\times \$ 10^{6}\right)$ & Average No. of BSSs & Average No. of batteries & Average No. of batteries per station \\
\hline 1 & 1.4215 & 2.5 & 74.7 & 30.91 \\
2 & 1.8676 & 2.9 & 136.7 & 48.575 \\
3 & 5114.1 & 6.1 & 503.8 & 82.71 \\
4 & 1.28828 & 15.3 & 1273.7 & 83.28 \\
5 & 6.3790 & 76.4 & 6356 & 83.21 \\
\hline
\end{tabular}




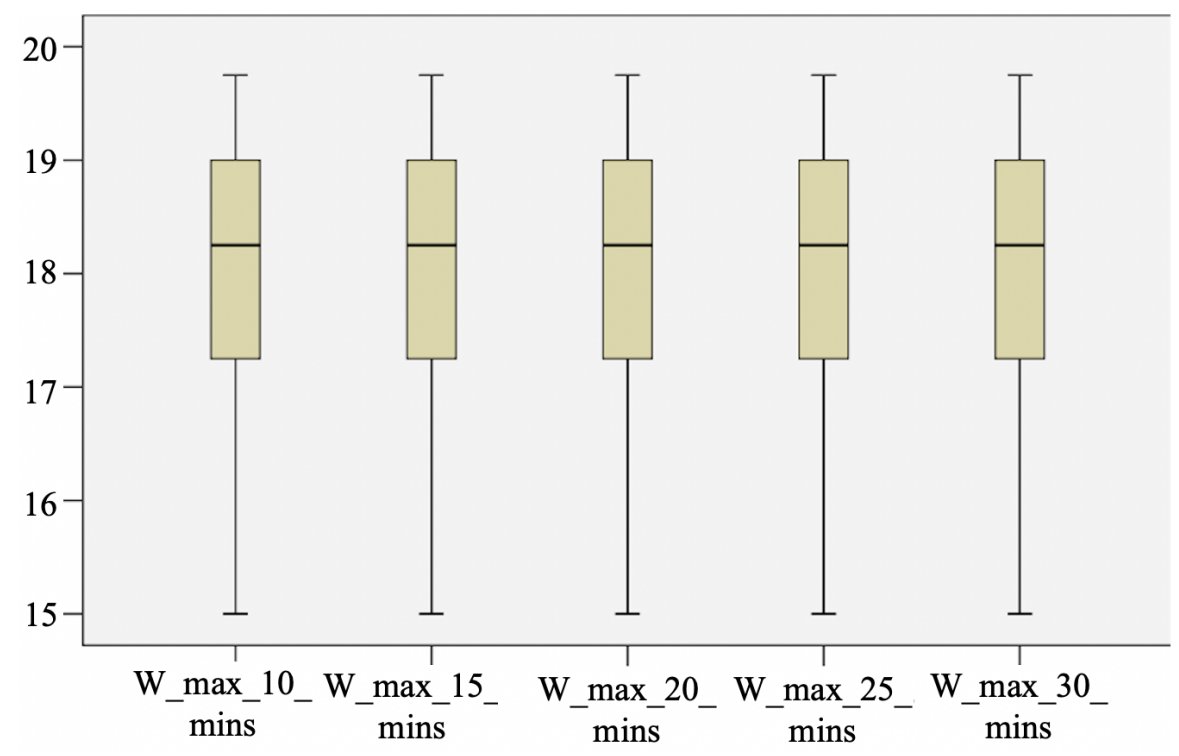

Figure 12. Aggregated arrival rate in stations for various $W_{\max }$ of Set 4 .

Figure 12 shows a boxplot of the aggregated arrivals across all stations, averaged over 10 instances for Set 4 . The results suggest that $W_{\max }$ does not affect the aggregate arrival rate in the stations. This result occurs because, for Set 4, regardless of any change in $W_{\max }$, the total power capacity in constraint (18) is the binding constraint and produces the dominant effect on the aggregate arrival rate of EVs.

TABLE 21. Total cost $(\times \$ 1,000)$ and number of stations for various $W_{\max }$.

\begin{tabular}{ccccccc}
\hline Set & 10 min. & 15 min. & 20 min. & 25 min. & 30 min. & No. of stations \\
\hline 1 & 1421.5 & 1392.8 & 1378.8 & 1366.9 & 1364.8 & 2.5 \\
2 & 1867.6 & 1822.8 & 1804.6 & 1788.5 & 1779.4 & 2.9 \\
3 & 5114.1 & 4986.1 & 4944 & 4902 & 4859.3 & 6.1 \\
4 & 12882.8 & 12562.2 & 12455.1 & 12348 & 12240.9 & 15.3 \\
5 & 63790 & 62194 & 61662 & 61130 & 60598 & 76.4 \\
\hline
\end{tabular}




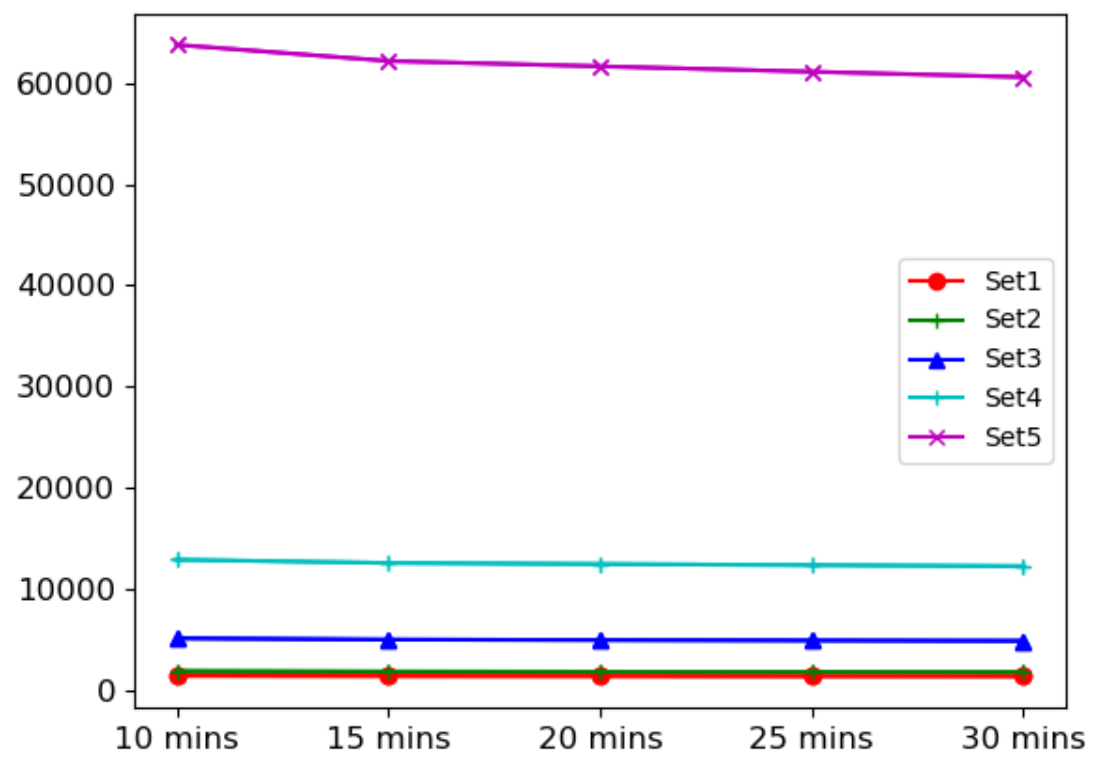

Figure 13. Total cost $(\times \$ 1,000)$ and number of stations as a function of $W_{\max }$.

Table 21 and Figure 13 show how the total network infrastructure cost changes when $W_{\max }$ varies from 10 to 30 minutes with five-minute increments. The costs from Sets 1 to 4 are much less than the cost from Set 5, which makes Sets 1 to 4 appear to be constant in Figure 13. Figure 15 shows the percent change in battery inventory as a function of $W_{\max }$. A relatively large increment in battery inventory occurs when $W_{\max }$ drops from 15 to 10 minutes. For instance, battery inventory in Set 4 increases only by $1.29 \%$ when $W_{\max }$ drops from 20 to 15 minutes, but the inventory increases by $3.7 \%$ when $W_{\max }$ drops from 15 to 10 minutes. As shown in Figure 14, the percent of the incremental cost of the infrastructure also grows steadily as $W_{\max }$ decreases, which is attributed to the fact that battery-inventory cost accounts for a major part of infrastructure expenses. 


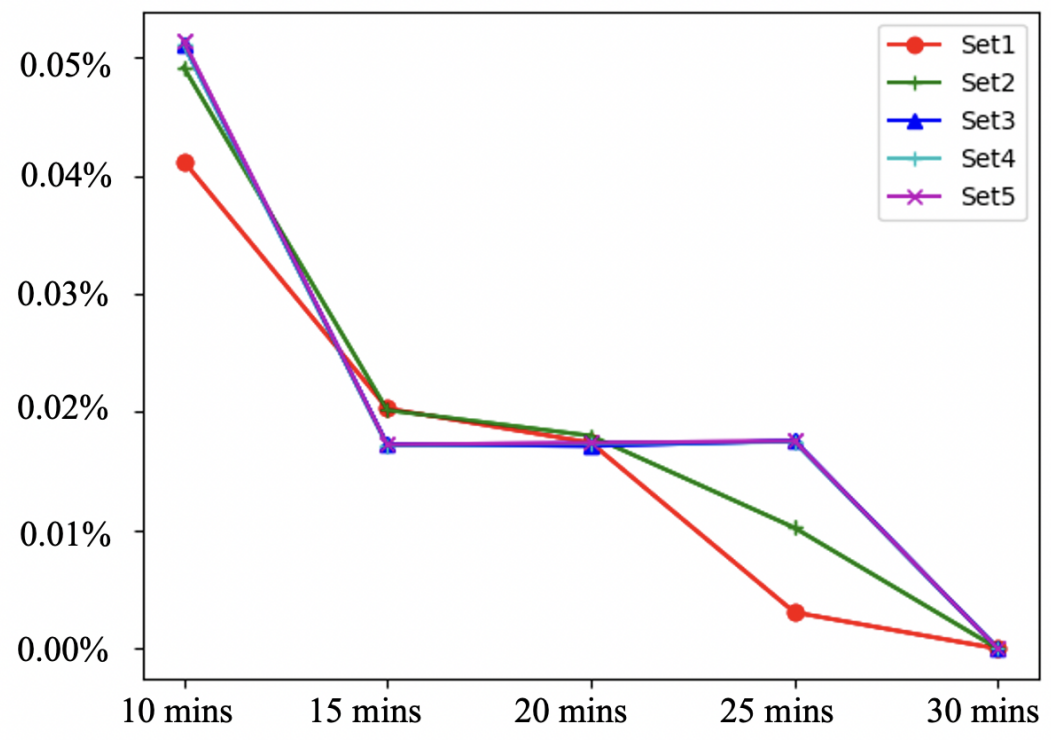

Figure 14. Percent change in cost for the OLIBSS-ST model as a function of $W_{\max }$.

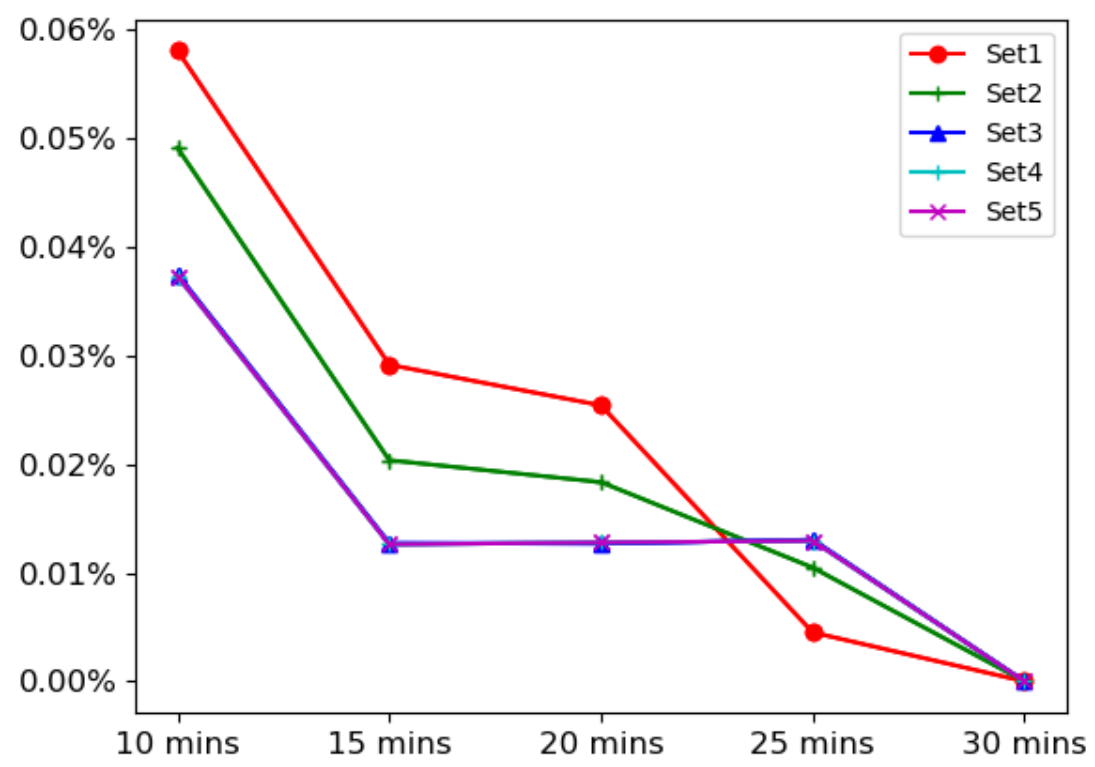

Figure 15. Percent change in battery inventory for the OLIBSS-ST model as a function of $W_{\max }$. 


\subsection{Numerical Results for the OLIHCS-SL Model}

We also tested the OLIHCS-SL model by using the 50 randomly generated instances. Table 22 displays the averaged results for $\gamma=0.2$ and $\beta=0.2$. The results show that, as network size increases, the average cost, number of batteries, and number of charging stations all increase. Also, because the cost of supercharging is much greater than the cost of batteries, the optimal solution exceeds the threshold value for $\gamma$ so that fewer EVs need superchargers, thus lowering the overall cost. In contrast, the optimal solution would meet the threshold value of 0.1 for $\beta$. Therefore, in contrast with the results given in Table 18, the OLIHCS-SL model uses more batteries (e.g., 52.6 vs. 58.9 for Set 1 and 4645.6 vs. 4916.8 for Set 2). Note that the increase in the number of batteries and extra superchargers requires more power. Therefore, given a fixed power capacity at any given station, the optimal solution tends to open more stations to cover all EVs in the area. As with the solutions of the OLIHCS-SL model when network size becomes large, the numbers of batteries and chargers per station stabilize because power capacity becomes a binding constraint.

TABLE 22. Averaged results for the OLIHCS-SL model $(\gamma=0.2, \beta=0.2)$.

\begin{tabular}{cccccc}
\hline Set & 1 & 2 & 3 & 4 & 5 \\
\hline Average cost $(\times \$ 1000)$ & 1463.9 & 1910.6 & 5175.3 & 13048.2 & 64446.4 \\
No. of stations & 2.5 & 2.9 & 6 & 15 & 74.8 \\
No. of batteries & 58.9 & 106.2 & 392.8 & 980.7 & 4916.8 \\
No. of batteries per station & 24.4 & 37.7 & 65.4 & 64.4 & 65.7 \\
No. of superchargers & 3.4 & 5.7 & 19.1 & 50.8 & 249.2 \\
No. of superchargers per station & 1.4 & 2.0 & 3.18 & 3.3 & 3.3 \\
Battery stockout probability & 0.12 & 0.13 & 0.15 & 0.15 & 0.15 \\
Waiting probability at supercharger & 0.17 & 0.18 & 0.17 & 0.16 & 0.16 \\
\hline
\end{tabular}

Table 23 shows the results of a sensitivity analysis of the OLIHCS-SL model in which $\gamma$ and $\beta$ are varied. With the highest service level for both battery swapping and supercharging, the network has the highest $(\gamma=0.05, \beta=0.05)$ total infrastructure cost. Furthermore, for fixed battery stockout probability $\gamma$ (queueing probability $\beta$ ), lower queueing probability $\beta$ (battery stockout probability $\gamma$ ) causes higher total cost because it requires more superchargers (battery inventory) to guarantee that the service level is met. In addition, by fixing $\gamma$, the total cost may 
not necessarily increase linearly in $\beta$. This appears in Figure 16 when $\gamma$ is set to 0.05. In addition, the combination of low $\beta$ and high $\gamma$ costs more than the combination of high $\beta$ and low $\gamma$. For example, Set 3 costs 5499.4 when $\gamma=0.1$ and $\beta=0.05$, which is more expensive than the total cost of 5443.5 when $\gamma=0.05$ and $\beta=0.1$. In addition, when secondary superchargers exist in a charging station, increasing $\gamma$ has less effect on the required number of charging stations because the superchargers can serve as a buffer. This is evinced by comparing Tables 10 and 8 . For example, for Set 4 , when $\gamma$ drops from 0.2 to 0.05 , the average number of stations increases only by 0.4 under the OLIHCS-SL model. However, under the OLIBSS-SL model, the average number of stations increases by 3.2 .

TABLE 23. Sensitivity analysis of cost of the OLIHCS-SL model $(\times 1000)$ and number of stations for various $\gamma$ and $\beta$.

\begin{tabular}{|c|c|c|c|c|c|c|c|c|}
\hline & \multicolumn{4}{|c|}{$\gamma=0.05$} & \multicolumn{4}{|c|}{$\gamma=0.1$} \\
\hline Set & $\beta=0.05$ & $\beta=0.1$ & $\beta=0.15$ & $\beta=0.2$ & $\beta=0.05$ & $\beta=0.1$ & $\beta=0.15$ & $\beta=0.2$ \\
\hline 1 & $1556.3(2.5)$ & $1522.8(2.5)$ & $1508.1(2.5)$ & $1501.8(2.5)$ & $1543(2.5)$ & $1511.9(2.5)$ & $1495.1(2.5)$ & $1481.8(2.5)$ \\
\hline 2 & $2047.6(2.9)$ & $2010.8(2.9)$ & $1918.6(2.9)$ & $1958.9(2.9)$ & $2023.7(2.9)$ & $1986.1(2.9)$ & $1962.6(2.9)$ & $1939.3(2.9)$ \\
\hline 3 & $5530(6.1)$ & $5443.5(6.1)$ & $5436.9(6.1)$ & $5400.5(6.1)$ & $5499.4(6.1)$ & $5388.2(6.1)$ & $5319(6.1)$ & $5252(6.1)$ \\
\hline 4 & $13942(15.4)$ & $13718.9(15.4)$ & $13709.3(15.4)$ & $13619(15.4)$ & $13847.3(15.4)$ & $13595.5(15.4)$ & $13410.1(15.4)$ & $13235.9(15.4)$ \\
\hline \multirow[t]{2}{*}{5} & $69006.4(76.8)$ & $67898.8(76.8)$ & $67852(76.8)$ & $67431.6(76.8)$ & $68491.2(76)$ & $67114.8(76)$ & $66299.6(76)$ & $65497.6(76)$ \\
\hline & \multicolumn{4}{|c|}{$\gamma=0.15$} & \multicolumn{4}{|c|}{$\gamma=0.2$} \\
\hline Set & $\beta=0.05$ & $\beta=0.1$ & $\beta=0.15$ & $\beta=0.2$ & $\beta=0.05$ & $\beta=0.1$ & $\beta=0.15$ & $\beta=0.2$ \\
\hline 1 & $1537.7(2.5)$ & $1505.2(2.5)$ & $1483.9(2.5)$ & $1470.6(2.5)$ & $1537.3(2.5)$ & $1501.4(2.5)$ & $1480.7(2.5)$ & $1463.9(2.5)$ \\
\hline 2 & $2020.6(2.9)$ & $1974(2.9)$ & $1939.4(2.9)$ & $1921.3(2.9)$ & $2020.6(2.9)$ & $1969.7(2.9)$ & $1936.4(2.9)$ & $1910.6(2.9)$ \\
\hline 3 & $5469.8(6)$ & $5342.9(6)$ & $5251.4(6)$ & $5197.1(6)$ & $5474(6)$ & $5337.5(6)$ & $5238.1(6)$ & $5175.3(6)$ \\
\hline 4 & $13839.3(15.1)$ & $13492.6(15.1)$ & $13271.9(15.1)$ & $13117.3(15.1)$ & $13796.5(15)$ & $13450.1(15)$ & $13180.2(15)$ & $13048.2(15)$ \\
\hline 5 & $68277.6(74.8)$ & 66714 (74.8) & $65568.4(74.8)$ & 64636 (74.8) & $68270(74.8)$ & $66438(74.8)$ & $65211.2(74.8)$ & $64446.4(74.8)$ \\
\hline
\end{tabular}




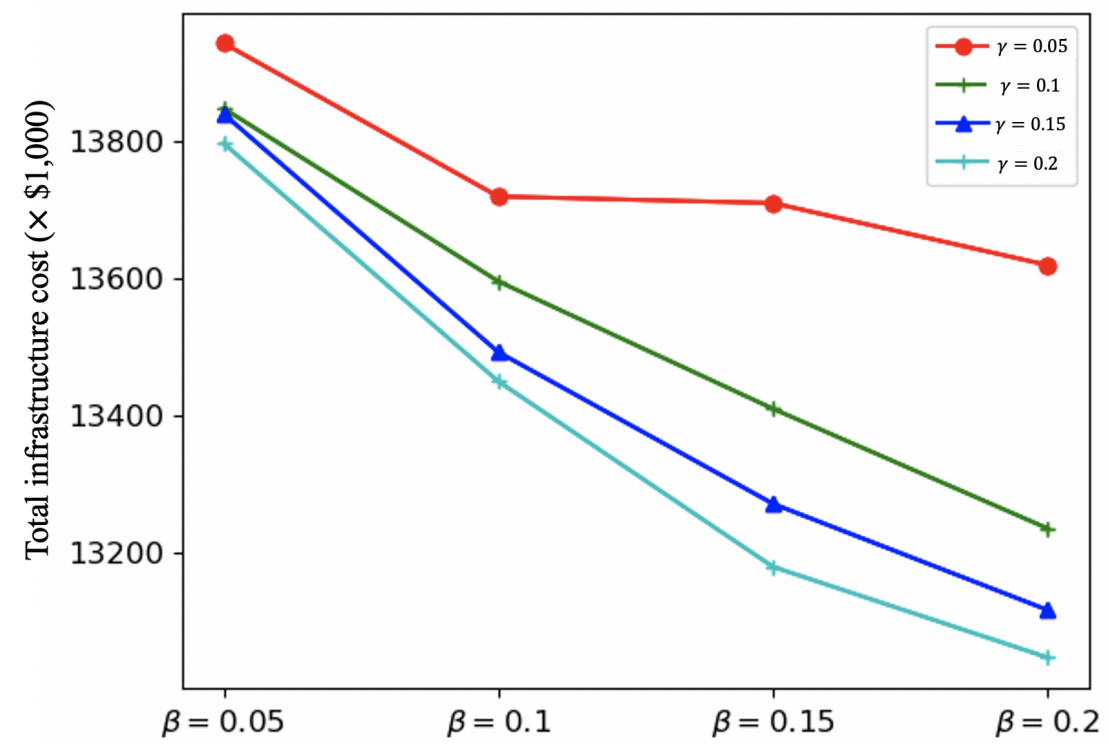

Figure 16. Total cost as a function of $\beta$ and $\gamma$ of Set 4 .

\subsection{Numerical Results for the OLIHCS-ST Model}

The OLIHCS-ST model was also tested with the same 50 instances. Table 24 shows the averaged results for $W_{\max }=10$ minutes. As expected, when the network size increases, the average cost, battery inventory, and number of charging stations all increase. Second, only one supercharger is required for each station for $W_{\max }=10$ minutes. In our setting, the supercharger needs half an hour to recharge the $24 \mathrm{kWh}$ battery, so the number of EVs being supercharged should be minimal in this circumstance. In other words, the OLIHCS-ST model prioritizes battery swapping over supercharging. Third, compared with the result of the OLIBSS-ST model (see Table 20) the number of spare batteries inventory is smaller. For instance, in Set 5 the number of batteries is 76.29 as per the OLIHCS-ST model as opposed to 83.21 as per the OLIBSS-ST model. This result is attributed to the superchargers, which can replace certain spare batteries while keeping the same service level.

TABLE 24. Averaged results for the OLIHCS-ST model with $W_{\max }=10$ minutes. 


\begin{tabular}{cccccc}
\hline Set & 1 & 2 & 3 & 4 & 5 \\
\hline Average cost $(\times \$ 1000)$ & 1452.1 & 1880.5 & 5091.1 & 12822.3 & 63506.8 \\
No. of stations & 2.5 & 2.9 & 6.1 & 15.3 & 76.4 \\
No. of batteries & 63 & 119.9 & 461.3 & 1166.7 & 5824.4 \\
No. of batteries per station & 26.13 & 42.7 & 75.74 & 76.29 & 76.29 \\
No. of superchargers & 2.5 & 2.9 & 6.1 & 15.3 & 76.4 \\
No. of superchargers per station & 1 & 1 & 1 & 1 & 1 \\
Battery stockout probability & 0.08 & 0.07 & 0.06 & 0.06 & 0.06 \\
Waiting probability at supercharger & 0.24 & 0.35 & 0.52 & 0.52 & 0.52 \\
\hline
\end{tabular}

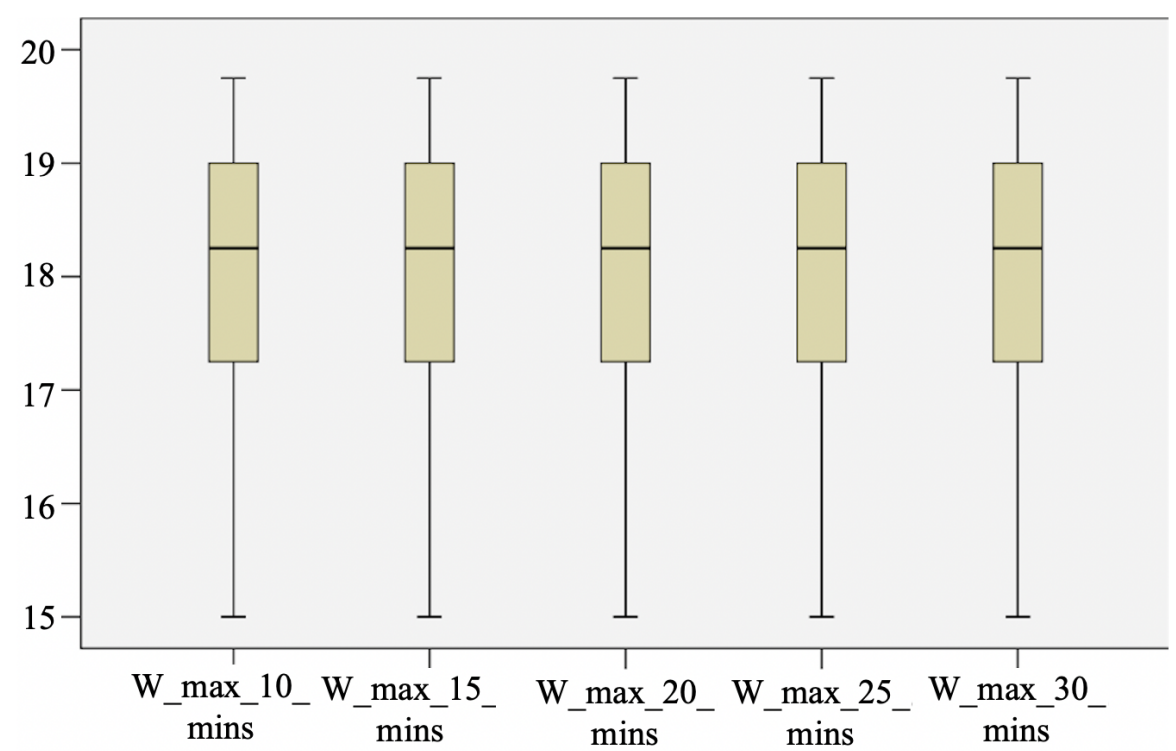

Figure 17. Aggregated arrival rate in stations for various $W_{\max }$ of Set 4 .

Figure 17 shows a boxplot of the aggregated arrivals across all stations, averaged over 10 instances in Set 4 . The results suggest that $W_{\max }$ does not affect the aggregate arrival rate in all stations, as is the case for the OLIBSS-ST model. As $W_{\text {max }}$ increases, more EVs are sent to the supercharging service. The secondary charging service serves as a buffer for the queue.

TABLE 25. Total cost $(\times \$ 1,000)$ and number of stations for various $W_{\max }$. 


\begin{tabular}{ccccccc}
\hline Set & 10 min. & 15 min. & 20 min. & 25 min. & 30 min. & No. of stations \\
\hline 1 & 1452.1 & 1403.8 & 1378.8 & 1365.9 & 1345.9 & 2.5 \\
2 & 1880.5 & 1813.7 & 1778.4 & 1752.8 & 1730.2 & 2.9 \\
3 & 5091.1 & 4896.6 & 4786.3 & 4698.8 & 4616.1 & 6.1 \\
4 & 12822.3 & 12330.3 & 12047.7 & 11832.6 & 11623.4 & 15.3 \\
5 & 63506.8 & 61054 & 59658.8 & 58568.8 & 57536 & 76.4 \\
\hline
\end{tabular}

Table 25 shows the results of a sensitivity analysis on optimal total costs with $W_{\text {max }}$ varied from 10 to 30 minutes. Two relatively-larger-cost increments occur when the allowable system time decreases from 30 to 25 minutes and from 15 to 10 minutes, respectively. For example, in Set 4 the total cost increases by $1.8 \%$ and by $4 \%$ when $W_{\max }$ drops from 30 to 25 minutes and from 15 to 10 minutes, respectively. These results are similar to those of the OLIBSS-ST model shown in Figure 14. Table 26 and Figure 18 show the average battery inventory each set as a function of system time. The number of batteries decreases as the allowable system time increases. When the sojourn-time constraint decreases from 30 to 25 minutes, the number of batteries increases significantly. For example, the number of batteries required in stations for Set 3 increases by $32.92 \%$ (as opposed to $35.78 \%$ and $36.02 \%$ for Sets 4 and 5, respectively).

TABLE 26. Average number of batteries for various $W_{\max }$.

\begin{tabular}{|c|c|c|c|c|c|c|c|c|c|c|}
\hline \multirow[t]{2}{*}{ Set } & \multicolumn{2}{|c|}{$10 \mathrm{~min}}$. & \multicolumn{2}{|c|}{$15 \mathrm{~min}}$. & \multicolumn{2}{|c|}{$20 \min }$. & \multicolumn{2}{|c|}{$25 \min }$. & \multicolumn{2}{|c|}{$30 \mathrm{~min}}$. \\
\hline & $\begin{array}{l}\text { Average } \\
\text { number of } \\
\text { batteries }\end{array}$ & $\begin{array}{l}\text { Average } \\
\text { number of } \\
\text { batteries } \\
\text { per station }\end{array}$ & $\begin{array}{l}\text { Average } \\
\text { number of } \\
\text { batteries }\end{array}$ & $\begin{array}{l}\text { Average } \\
\text { number of } \\
\text { batteries } \\
\text { per station }\end{array}$ & $\begin{array}{l}\text { Average } \\
\text { number of } \\
\text { batteries }\end{array}$ & $\begin{array}{l}\text { Average } \\
\text { number of } \\
\text { batteries } \\
\text { per station }\end{array}$ & $\begin{array}{l}\text { Average } \\
\text { number of } \\
\text { batteries }\end{array}$ & $\begin{array}{l}\text { Average } \\
\text { number of } \\
\text { batteries } \\
\text { per station }\end{array}$ & $\begin{array}{l}\text { Average } \\
\text { number of } \\
\text { batteries }\end{array}$ & $\begin{array}{l}\text { Average } \\
\text { number of } \\
\text { batteries } \\
\text { per station }\end{array}$ \\
\hline 1 & 63 & 25.2 & 56.1 & 22.4 & 47.4 & 19 & 44.9 & 18 & 36.9 & 14.8 \\
\hline 2 & 119.9 & 41.3 & 106.5 & 36.7 & 88.6 & 30.6 & 79.8 & 27.5 & 65 & 22.4 \\
\hline 3 & 461.3 & 75.6 & 394.3 & 64.6 & 337.4 & 55.3 & 270.9 & 44.4 & 203.8 & 33.4 \\
\hline 4 & 1166.7 & 76.2 & 994.2 & 65 & 843.9 & 55.1 & 689.1 & 45 & 507.5 & 33.2 \\
\hline 5 & 5824.4 & 76.2 & 4920 & 65 & 4220.4 & 55.2 & 3460.4 & 45.3 & 2544 & 33.3 \\
\hline
\end{tabular}




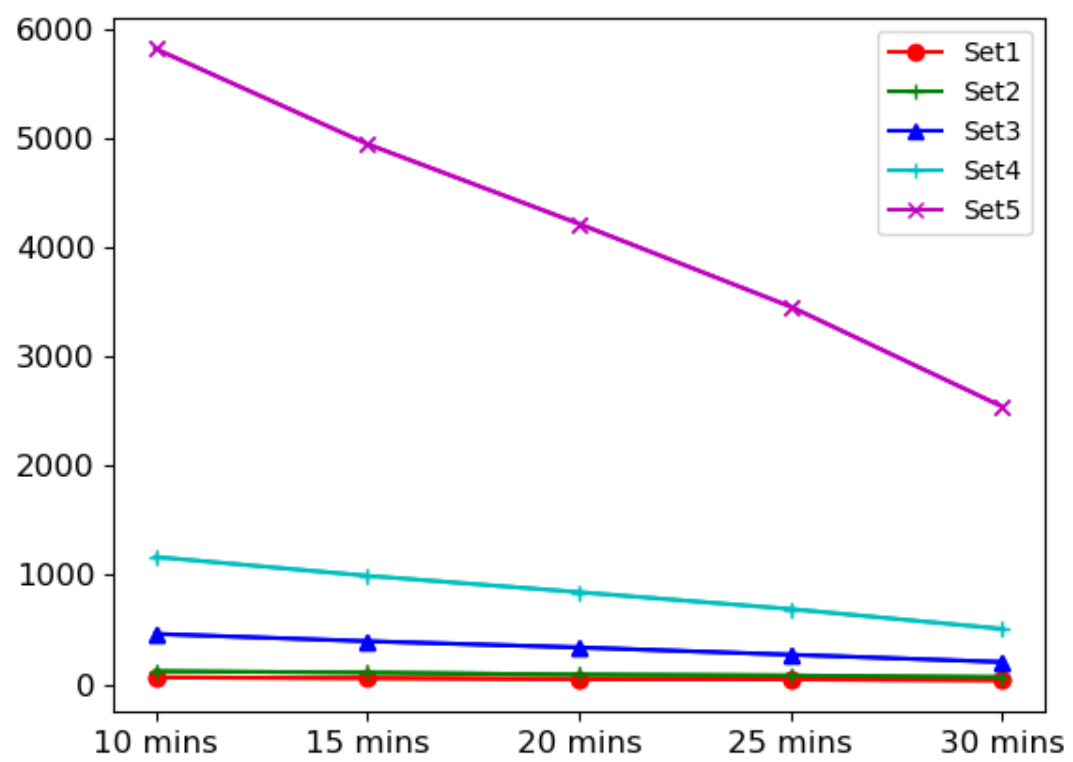

Figure 18. Average number of batteries for various $W_{\max }$.

Table 27 and Figure 19 show the number of superchargers per station for various $W_{\max }$. The number of superchargers decreases as $W_{\max }$ decreases because superchargers need 30 minutes to finish the charging process. A huge increase in superchargers occurs when $W_{\max }$ goes from 10 to 15 minutes. For example, the number of superchargers increases by $100 \%, 103.92 \%$, and $106.28 \%$ when $W_{\max }$ increases from 10 to 15 minutes for Sets 3-5, respectively.

TABLE 27. Average number of superchargers for various $W_{\max }$.

\begin{tabular}{|c|c|c|c|c|c|c|c|c|c|c|}
\hline \multirow[t]{2}{*}{ Set } & \multicolumn{2}{|c|}{$10 \mathrm{~min}}$. & \multicolumn{2}{|c|}{$15 \mathrm{~min}}$. & \multicolumn{2}{|c|}{$20 \mathrm{~min}}$. & \multicolumn{2}{|c|}{$25 \mathrm{~min}}$. & \multicolumn{2}{|c|}{$30 \mathrm{~min}}$. \\
\hline & $\begin{array}{l}\text { Average } \\
\text { number of } \\
\text { superchargers }\end{array}$ & $\begin{array}{l}\text { Average } \\
\text { number of } \\
\text { superchargers } \\
\text { per station }\end{array}$ & $\begin{array}{l}\text { Average } \\
\text { number of } \\
\text { superchargers }\end{array}$ & $\begin{array}{l}\text { Average } \\
\text { number of } \\
\text { superchargers } \\
\text { per station }\end{array}$ & \begin{tabular}{|l} 
Average \\
number of \\
superchargers
\end{tabular} & \begin{tabular}{|l|} 
Average \\
number of \\
superchargers \\
per station
\end{tabular} & $\begin{array}{l}\text { Average } \\
\text { number of } \\
\text { superchargers }\end{array}$ & $\begin{array}{l}\text { Average } \\
\text { number of } \\
\text { superchargers } \\
\text { per station }\end{array}$ & $\begin{array}{l}\text { Average } \\
\text { number of } \\
\text { superchargers }\end{array}$ & $\begin{array}{l}\text { Average } \\
\text { number of } \\
\text { superchargers } \\
\text { per station }\end{array}$ \\
\hline 1 & 2.5 & 1 & 2.5 & 1 & 3.3 & 1.3 & 3.4 & 1.4 & 4.2 & 1.7 \\
\hline 2 & 2.9 & 1 & 3.5 & 1.2 & 5.5 & 1.9 & 6.3 & 2.2 & 8.1 & 2.8 \\
\hline 3 & 6.1 & 1 & 12.2 & 2 & 18.6 & 3 & 27 & 4.4 & 35.6 & 5.9 \\
\hline 4 & 15.3 & 1 & 31.2 & 2 & 48.3 & 3.2 & 67.6 & 4.4 & 91.2 & 6 \\
\hline 5 & 76.4 & 1 & 157.6 & 2 & 240.4 & 3.2 & 334.4 & 4.4 & 454 & 6 \\
\hline
\end{tabular}




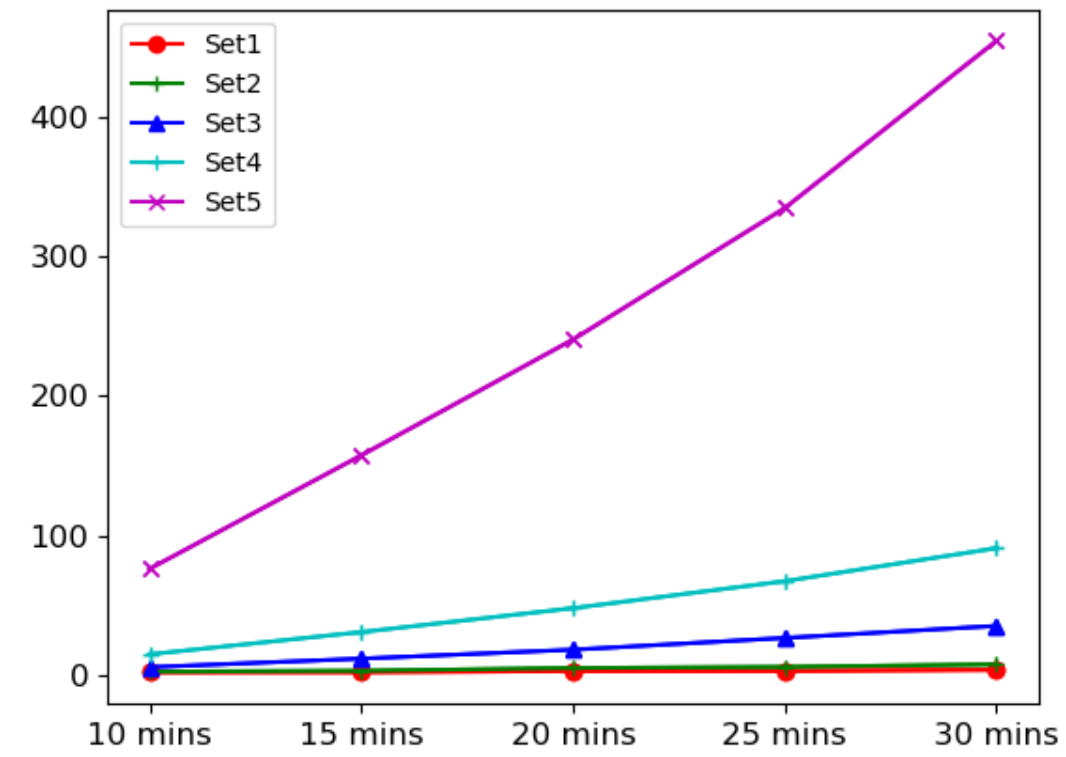

Figure 19. Average number of superchargers as a function of $W_{\max }$.

\subsection{Case Study for Mid-Ohio Region}

We apply the proposed OLIHCS-SL model, OLIHCS-ST model, and metaheuristic algorithm to a case study of the central Ohio regional network available through the Mid-Ohio Regional Planning Commission (http://www.morpc.org). The data involve 714000 households, 2 million light-duty vehicles in seven counties, and 2.5 million personal trips in 2015. We use $3.3 \% \mathrm{EV}$ penetration rate as suggested in Ref. [82]. As illustrated in Table 28, each data entry is a tour consisting of at least one trip. Each trip has a designated origin and destination TAZ with known distance. For example, the table displays a direct trip for household 1 traveling from TAZ 1 to 4 and an indirect trip for household 2 traveling from TAZ 14 to 27 with an intermediate stop at TAZ 30. Furthermore, because the case study focuses on siting charging stations with easy accessibility, we choose as candidate locations nonresidential areas such as retail stores, shopping malls, office complexes, and gas stations along the highway. 
TABLE 28. Sample data for case study (note: $i, k$, and $l$ are TAZ indices).

\begin{tabular}{ccccccc}
\hline Household No. & Start TAZ $i$ & End TAZ $l$ & Intermediate stop (TAZ No.) & Distance from $i$ to $l$ & Distance from $i$ to $k$ & Distance from $k$ to $l$ \\
\hline 1 & 1 & 4 & & 13 & & \\
2 & 14 & 27 & 30 & 19 & 15 & 21 \\
3 & 36 & 79 & 45 & 12 & 6 & 7 \\
\hline
\end{tabular}

The EV charging-demand forecasting method in Section 5.1 is used to estimate the EV arrival rate $\lambda_{i}$ for each TAZ. Figure 20(a) shows a boxplot for the calculated arrival rates for all TAZs in the case study. These results suggest that, for most TAZs, the arrival rate $\lambda_{i}$ is $1-4$ vehicles/hour. Conversely, Kuby and Lim [26] conclude that about $83.4 \%$ of alternative-fuel drivers refill their vehicle when the energy source is less than $3 / 8$ of full capacity. Therefore, all tours with a distance exceeding $5 / 8$ of the full battery range could translate into a potential charging demand at the destination. In general, it is reasonable to assume that EVs may have some energy left in their batteries before finding a service station-our experiment sets this buffer at $1 / 8$ of the full battery capacity. Therefore, the maximum allowable range in this case study is $2 / 8$ of the full battery range. Similar to the randomly generated test instances, the Nissan Leaf is again considered in the case study and the maximum allowable range is 30 miles (or $48 \mathrm{~km}$ ) for the $24 \mathrm{kWh}$ battery. 


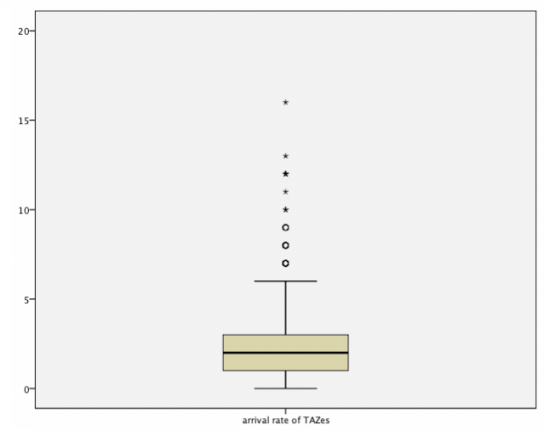

a. Boxplot of arrival rate in TAZes

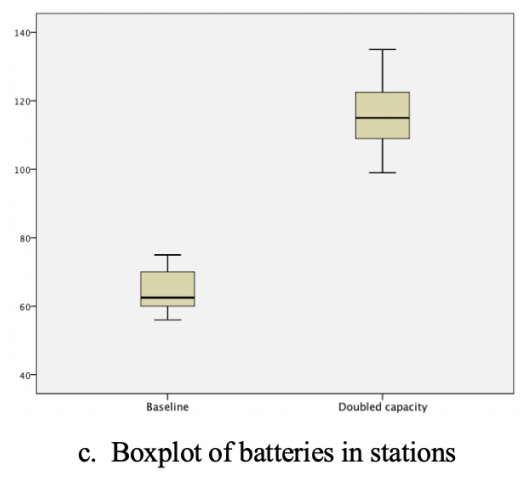

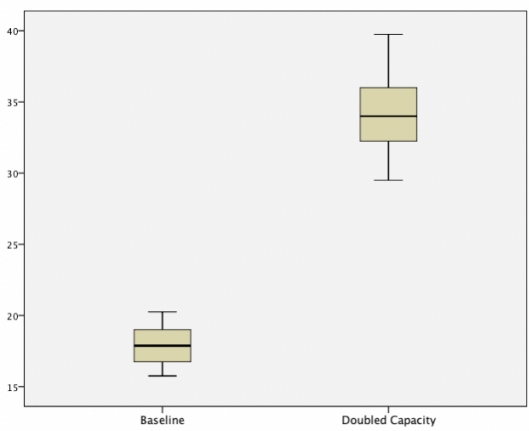

b. Boxplot of aggregated arrival rate in stations

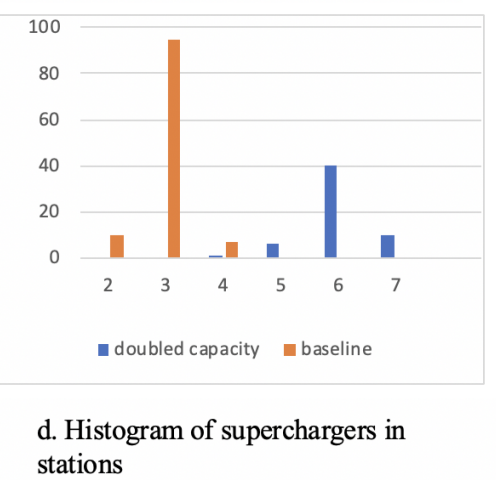

Figure 20. Results for Mid-Ohio case with the OLIHCS-SL model.

We ran the OLIHCS-SL model on the Mid-Ohio case with $\gamma=0.2$ and $\beta=0.2$ Figures 20(b)-20(d) show the results for arrival rates covered at all open stations, the number of batteries, and the number of superchargers, respectively. In particular, we run two scenarios: one with a baseline power capacity and another with a doubled power capacity. The model suggests opening 112 stations in the baseline case, which decreases to 57 stations under the doubled-power-capacity case. Furthermore, Figure 20(b) shows the distribution of the responsible arrival rates for all 112 stations under the baseline capacity with an average of $18 \mathrm{EVs}$ per hour. Under the doubled capacity, the average EV arrival rate is approximately 34 cars per hour. Conversely, for the baseline case, Figure 20(c) shows the battery inventories for all stations, with most stations having 55-75 batteries in stock. When the power capacity is doubled, the average battery inventory is about 118 batteries. Finally, Figure 20(d) suggests that the majority of stations with three superchargers installed needs to increase to six superchargers when the power capacity is doubled. 
We also ran the OLIBSS-ST model on the Mid-Ohio case for $W_{\max }=10$ minutes. The results suggest opening 128 stations in the baseline case, which decreases to 64 stations for the doubled-power-capacity case. The average EV arrival rate is approximately $17 \mathrm{EVs}$ per hour for the baseline case and $34 \mathrm{EVs}$ per hour for the doubled-power-capacity case. The numbers of batteries and chargers per station are 72.4 and 1, respectively, for the baseline case. When the power capacity is doubled, the numbers of batteries and chargers per station increase to 134.9 and 2 , respectively.

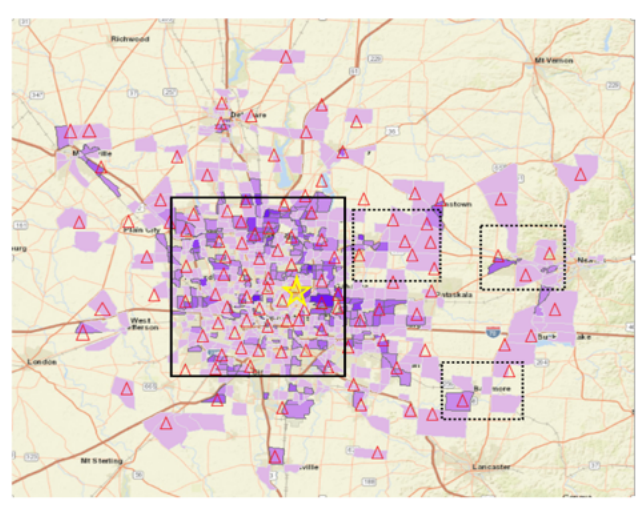

a. Map of the charging stations for the central Ohio case

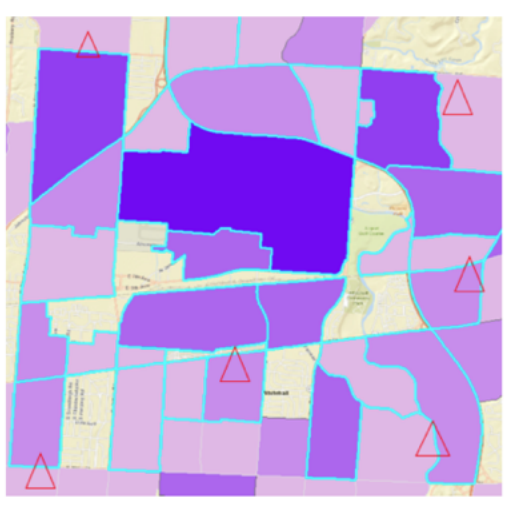

b. Zoomed map for charging stations in the Columbus airport area

Figure 21. Results for Mid-Ohio case with the OLIHCS-SL model.

Figure 21(a) shows the map of all 112 opened stations under the baseline-capacity case. The darker colors represent a greater arrival rate for the given TAZ. The results show that most stations are placed in the Columbus metropolitan area (rectangular boxes with solid lines). In addition, Figure 21(a) reveals three satellite areas (rectangular boxes with dashed lines), each of which contains two to three stations. In particular, the area with the highest EV arrival rates is marked with a star. An expanded view of this area around the Columbus airport is shown in Figure 21(b). This area accepts 110 EVs per hour from 27 TAZs. As a result, six stations are established, as shown in Figure 21(b). These stations meet the demand from EV arrival rates of $17,18,20,18,18$, and $19 \mathrm{EV} /$ hour by using $61,66,74,66$, 66 , and 70 batteries, respectively. In addition, each of the six stations has three 
superchargers installed. The OLIBSS-ST model sets up one more station to meet the demand from EV arrival rates of 15, 17, 18, 19, 18, 18, and $19 \mathrm{EV} /$ hour by using $64,72,76,80,76,76$, and 80 batteries, respectively. Each of these seven stations has one supercharger installed.

TABLE 29. Results for the OLIHCS-SL model for various charging rates.

\begin{tabular}{lccccccc}
\hline & Scenario & $\gamma$ & $\beta$ & Stations & Average $s_{j} \mathrm{~s}$ & Average $m_{j} \mathrm{~s}$ & Cost $(\$ 1,000)$ \\
\hline \multirow{2}{*}{ Baseline } & 1 & 0.2 & 0.2 & 112 & 64.8 & 3.0 & 96,103 \\
$\mu_{b}=0.25$ & 2 & 0.2 & 0.1 & 112 & 64.6 & 3.9 & 100,482 \\
$P_{b}=10 \mathrm{~kW}$ & 3 & 0.1 & 0.2 & 113 & 73.2 & 2.2 & 98,935 \\
& 4 & 0.1 & 0.1 & 113 & 75.6 & 2.4 & 101,850 \\
\hline & 1 & 0.2 & 0.2 & 112 & 36.7 & 2.7 & 71,157 \\
$\mu_{b}=0.5$ & 2 & 0.2 & 0.1 & 112 & 37.2 & 3.1 & 73,565 \\
$P_{b}=20 \mathrm{~kW}$ & 3 & 0.1 & 0.2 & 113 & 40.8 & 1.8 & 71,386 \\
& 4 & 0.1 & 0.1 & 113 & 43.7 & 1.8 & 73,680 \\
$\mu_{b}=1$ & 1 & 0.2 & 0.2 & 112 & 24.3 & 1.8 & 59,709 \\
$P_{b}=40 \mathrm{~kW}$ & 2 & 0.2 & 0.1 & 112 & 25.6 & 2 & 61,736 \\
& 3 & 0.1 & 0.2 & 113 & 24.0 & 1.2 & 60,177 \\
\hline
\end{tabular}

Finally, we project the technological changes for increased charging rate $\mu_{b}$ for level-2 chargers for swapped batteries and investigate how the increase in $\mu_{b}$ affects the numbers of stations opened, the number of spare batteries, and the number of superchargers installed. Three station-configuration scenarios are created: $\mu_{b}=0.25$ (baseline), $\mu_{b}=0.5$, and $\mu_{b}=1$. For each scenario, we vary both $\gamma$ and $\beta$ from 0.1 to 0.2 , for a total of four combinations. Table 29 shows that increasing $\mu_{b}$ has minimal effect on the number of stations opened, which remains at 112 or 113 for the entire service network. The average number decreases for both spare batteries and superchargers, which results in a lower total cost. Finally, of the four combinations, $(\gamma, \beta)=(0.2,0.2)$ and $(\gamma, \beta)=(0.1,0.1)$ yield the lowest and highest total cost, respectively, and the costs for $(\gamma, \beta)=(0.2,0.1)$ and $(\gamma, \beta)=(0.1,0.2)$ are 
in the middle with $(\gamma, \beta)=(0.2,0.1)$ being higher. The latter happens because more superchargers need to be installed for smaller $\beta$, thus leading to higher cost.

TABLE 30. Results for the OLIHCS-ST model for various charging rates.

\begin{tabular}{lcccccc}
\hline & Scenario & $W_{\text {max }}$ & Stations & Average $s_{j} \mathrm{~s}$ & Average $m_{j} \mathrm{~s}$ & Cost $(\$ 1,000)$ \\
\hline Baseline & 1 & 10 & 128 & 73 & 1 & 106,876 \\
$\mu_{b}=0.25$ & 2 & 15 & 128 & 62 & 2 & 102,780 \\
$P_{b}=10 \mathrm{~kW}$ & 3 & 20 & 128 & 53.2 & 2.9 & 100,492 \\
& 4 & 25 & 128 & 43.6 & 4.2 & 98,792 \\
& 5 & 30 & 128 & 31.8 & 5.6 & 96,283 \\
$\mu_{b}=0.5$ & 1 & 10 & 128 & 39.4 & 1 & 76,832 \\
$P_{b}=20 \mathrm{~kW}$ & 3 & 15 & 128 & 37.5 & 1 & 75,068 \\
& 4 & 20 & 128 & 36.9 & 1 & 74,508 \\
& 5 & 30 & 128 & 36.1 & 1 & 74,172 \\
& 1 & 10 & 128 & 22.2 & 1 & 73,813 \\
$\mu_{b}=1$ & 2 & 15 & 128 & 20.9 & 1 & 61,376 \\
$P_{b}=40 \mathrm{~kW}$ & 3 & 20 & 128 & 20.2 & 1 & 50,228 \\
& 4 & 25 & 128 & 20.2 & 1 & 59,612 \\
& 5 & 30 & 128 & 20.2 & 1 & 59,612 \\
\hline
\end{tabular}

The same projection was made for the OLIHCS-ST model. For each charging rate given above, $W_{\max }$ is set at four levels: 10, 15, 20, and 25 minutes. The results shown in Table 30 are similar to those of Table 29; that is, 128 stations remain open for all scenarios, and the number of batteries and the total network cost decrease as $\mu_{b}$ increases. In particular, when the battery charging rate starts to catch up with the charging rate of superchargers, the cost advantage of batteries makes stations allocate more batteries than superchargers, which ultimately reduces the number of superchargers to one per station. Finally, when $\mu_{b}=1$, changing from $W_{\max }=20$ minutes to $W_{\max }=25$ minutes does not affect the configuration of stations because the time difference is trivial compared with the total time an EV spends in the charging stations. 


\section{CHAPTER 6}

\section{CONCLUSION AND FUTURE RESEARCH}

In this dissertation, we establish and verify that the process for swapping EV batteries follows an $\mathrm{M} / \mathrm{G} / \mathrm{s} / \mathrm{s}$ and $\mathrm{M} / \mathrm{G} / \mathrm{s} / \infty$ queueing model and we use the Erlang function to combine queuing theory and inventory control. To reduce EV-range anxiety, two types of novel battery service stations are proposed for EVs. The first type of station offers only a battery swapping service. Two models, the optimal location and inventory model for BSSs with service-level constraint (OLIBSS-SL) and the optimal location and inventory model for BSSs with service time constraint (OLIBSS-ST), are formulated to study (i) station location and battery inventory for target service levels and (ii) total sojourn-time constraints, respectively. Both models minimize the total infrastructure cost and battery procurement cost. The second type of battery service station offers not only a battery swapping service but also an on-site supercharging service. As for the second battery service station, we construct an optimal location and inventory model for a battery swapping and supercharging stations with service-level constraint (OLIHCS-SL) and an optimal location and inventory model for battery swapping and supercharging stations with service time constraint (OLIHCS-ST) to study service level and total sojourn-time constraints.

All proposed models minimize total infrastructure cost, battery cost, and supercharger cost. By studying the mathematical properties of the Erlang function, we develop heuristic algorithms to solve the four proposed models. The Erlang function is a nonlinear function with decision variables embedded in complicated factorial and power-law functions. Traditional solvers are thus unable to solve these problems or give global optimal solutions, which prevents the Erlang function from 
being applied to large-scale applications. The proposed algorithms can significantly simplify the model and save computational time. For the second type of battery-service station, we construct a case study based on vehicle displacement data from the Mid-Ohio region. Given that the data are based on TAZs, we construct a shortest-path problem to estimate the EV-charging demand for each TAZ. The proposed models and algorithms are then used for other service providers who wish to take queueing effect into consideration for decisions regarding where to locate battery-service stations. As a result, the heuristic algorithms solve the proposed models more rapidly and give more accurate results. 


\section{REFERENCES}

[1] L. Chapman, "Transport and climate change: a review," Journal of transport geography, vol. 15, no. 5, pp. 354-367, 2007.

[2] H.-Y. Mak, Y. Rong, and Z.-J. M. Shen, "Infrastructure Planning for Electric Vehicles with Battery Swapping," Management Science, vol. 59, no. 7, pp. 1557-1575, Jul. 2013.

[3] P. M. R. Almeida, F. J. Soares, and J. A. P. Lopes, "Impacts of Large-Scale Deployment of Electric Vehicles in the Electric Power System," in Electric Vehicle Integration into Modern Power Networks. New York, NY: Springer New York, Oct. 2012, pp. 203-249.

[4] N. L. Website, "Range and charging," https://www.nissan.co.uk/vehicles/new-vehicles/leaf/charging-range.html, accessed June 26, 2016.

[5] D. Dauer and C. M. Flath, "Simulation-based Evaluation of Battery Switching Stations for Electric Vehicles," Simulation, 2015.

[6] S. Agassi, "World Without Oil: Better Place Builds a Future for Electric Vehicles ( Innovations Case Narrative: Better Place)," Innovations: Technology, Governance, Globalization, vol. 4, no. 4, pp. 125-140, Oct. 2009.

[7] X. Tan, B. Sun, and D. H. K. Tsang, "Queueing network models for electric vehicle charging station with battery swapping," in 2014 IEEE International Conference on Smart Grid Communications (SmartGridComm). IEEE, 2014, pp. $1-6$.

[8] B. Avci, K. Girotra, and S. Netessine, "Electric Vehicles with a Battery Switching Station: Adoption and Environmental Impact," Management Science, vol. 61, no. 4, pp. 772-794, Apr. 2015. 
[9] J. Jung, J. Y. Chow, R. Jayakrishnan, and J. Y. Park, "Stochastic dynamic itinerary interception refueling location problem with queue delay for electric taxi charging stations," Transportation Research Part C: Emerging Technologies, vol. 40, pp. 123-142, 2014.

[10] M. B. Arias and S. Bae, "Electric vehicle charging demand forecasting model based on big data technologies," Applied energy, vol. 183, pp. 327-339, 2016.

[11] M. Hosseini and S. MirHassani, "Refueling-station location problem under uncertainty," Transportation Research Part E: Logistics and Transportation Review, vol. 84, pp. 101-116, 2015.

[12] M. Xu, Q. Meng, K. Liu, and T. Yamamoto, "Joint charging mode and location choice model for battery electric vehicle users," Transportation Research Part B: Methodological, vol. 103, pp. 68-86, 2017.

[13] M. Majidpour, C. Qiu, P. Chu, H. R. Pota, and R. Gadh, "Forecasting the ev charging load based on customer profile or station measurement?" Applied energy, vol. 163, pp. 134-141, 2016.

[14] D. Pevec, J. Babic, M. A. Kayser, A. Carvalho, Y. Ghiassi-Farrokhfal, and V. Podobnik, "A data-driven statistical approach for extending electric vehicle charging infrastructure," International journal of energy research, vol. 42, no. 9, pp. 3102-3120, 2018.

[15] C. Wang, J. Yang, N. Liu, and Y. Mao, "Study on siting and sizing of battery-switch station," in Electric Utility Deregulation and Restructuring and Power Technologies (DRPT), 2011 4th International Conference on. IEEE, 2011, pp. 657-662.

[16] F. Pan, R. Bent, A. Berscheid, and D. Izraelevitz, "Locating phev exchange stations in v2g," in Smart Grid Communications (SmartGridComm), 2010 First IEEE International Conference on. IEEE, 2010, pp. 173-178. 
[17] R. S. Widrick, S. G. Nurre, and M. J. Robbins, "Optimal policies for the management of an electric vehicle battery swap station," Transportation Science, vol. 52, no. 1, pp. 59-79, 2016.

[18] B. Sun, X. Sun, D. H. Tsang, and W. Whitt, "Optimal battery purchasing and charging strategy at electric vehicle battery swap stations," European Journal of Operational Research, 2019.

[19] H. Sun, J. Yang, and C. Yang, "A robust optimization approach to multi-interval location-inventory and recharging planning for electric vehicles," Omega, vol. 86, pp. 59-75, 2019.

[20] T. Raviv, "The battery switching station scheduling problem," Operations Research Letters, vol. 40, no. 6, pp. 546-550, Nov. 2012.

[21] L. Xinyi, L. Nian, H. Yangqi, Z. Jianhua, and Z. Nan, "Optimal configuration of EV battery swapping station considering service availability," in 2014 International Conference on Intelligent Green Building and Smart Grid (IGBSG). IEEE, 2014, pp. 1-5.

[22] H. Yudai and K. Osamu, "A safety stock problem in battery switch stations for electric vehicles," Proc of The Eighth International Symposium on Operations Research and Its Applications (ISORA'09), 2009.

[23] B. Avci, K. Girotra, and S. Netessine, "Electric Vehicles with a Battery Switching Station: Adoption and Environmental Impact," SSRN Electronic Journal, Oct. 2013.

[24] J. J. Jamian, M. W. Mustafa, H. Mokhlis, and M. A. Baharudin, "Simulation study on optimal placement and sizing of Battery Switching Station units using Artificial Bee Colony algorithm," International Journal of Electrical Power \& Energy Systems, vol. 55, pp. 592-601, Feb. 2014.

[25] M. J. Hodgson, "A flow-capturing location-allocation model," Geographical Analysis, vol. 22, no. 3, pp. 270-279, 1990. 
[26] M. Kuby and S. Lim, "The flow-refueling location problem for alternative-fuel vehicles," Socio-Economic Planning Sciences, vol. 39, no. 2, pp. 125-145, Jun. 2005.

[27] Y.-W. Wang and C.-C. Lin, "Locating road-vehicle refueling stations," Transportation Research Part E: Logistics and Transportation Review, vol. 45, no. 5, pp. 821-829, Sep. 2009.

[28] M. Armstrong, C. El Hajj Moussa, J. Adnot, A. Galli, and P. Riviere, "Optimal recharging strategy for battery-switch stations for electric vehicles in France," Energy Policy, vol. 60, pp. 569-582, Sep. 2013.

[29] F. Lu and G. Hua, "A location-sizing model for electric vehicle charging station deployment based on queuing theory," in Logistics, Informatics and Service Sciences (LISS), 2015 International Conference on. IEEE, 2015, pp. 1-5.

[30] T. Sweda and D. Klabjan, "An agent-based decision support system for electric vehicle charging infrastructure deployment," 2011 IEEE Vehicle Power and Propulsion Conference (VPPC), pp. 1-5, 2011.

[31] T. D. Chen, K. M. Kockelman, M. Khan et al., "The electric vehicle charging station location problem: a parking-based assignment method for seattle," in Transportation Research Board 92nd Annual Meeting, vol. 340, 2013, pp. $13-1254$.

[32] S. J. ERLEBACHER and R. D. MELLER, "The interaction of location and inventory in designing distribution systems," IIE Transactions, vol. 32, no. 2, pp. 155-166, Feb. 2000.

[33] P. A. Miranda and R. A. Garrido, "Incorporating inventory control decisions into a strategic distribution network design model with stochastic demand," Transportation Research Part E: Logistics and Transportation Review, vol. 40, no. 3, pp. 183-207, May 2004. 
[34] K. Sourirajan, L. Ozsen, and R. Uzsoy, "A single-product network design model with lead time and safety stock considerations," IIE Transactions, vol. 39, no. 5, pp. 411-424, 2007.

[35] E. Aghezzaf, "Capacity planning and warehouse location in supply chains with uncertain demands," Journal of the Operational Research Society, vol. 56, no. 4, pp. 453-462, Oct. 2004.

[36] L. K. Nozick, "The fixed charge facility location problem with coverage restrictions," Transportation Research Part E: Logistics and Transportation Review, vol. 37, no. 4, pp. 281-296, Aug. 2001.

[37] L. K. Nozick and M. A. Turnquist, "Inventory, transportation, service quality and the location of distribution centers," European Journal of Operational Research, vol. 129, no. 2, pp. 362-371, Mar. 2001.

[38] M. F. Candas and E. Kutanoglu, "Benefits of considering inventory in service parts logistics network design problems with time-based service constraints," IIE Transactions, vol. 39, no. 2, pp. 159-176, Feb. 2007.

[39] M. S. Daskin, C. R. Coullard, and Z.-J. M. Shen, "An inventory-location model: Formulation, solution algorithm and computational results," Annals of operations research, vol. 110, no. 1-4, pp. 83-106, 2002.

[40] Z.-J. M. Shen, C. Coullard, and M. S. Daskin, "A Joint Location-Inventory Model," Transportation Science, vol. 37, no. 1, pp. 40-55, Feb. 2003.

[41] H. E. Romeijn, J. Shu, and C.-P. Teo, "Designing two-echelon supply networks," European Journal of Operational Research, vol. 178, no. 2, pp. 449-462, Apr. 2007.

[42] H.-Y. Mak and Z.-J. M. Shen, "A two-echelon inventory-location problem with service considerations," Naval Research Logistics (NRL), vol. 56, no. 8, pp. 730-744, Dec. 2009. 
[43] F. You and I. E. Grossmann, "Integrated multi-echelon supply chain design with inventories under uncertainty: MINLP models, computational strategies," AIChE Journal, pp. NA-NA, 2009.

[44] J. Ridlehoover, "APPLYING MONTE CARLO SIMULATION AND RISK ANALYSIS TO THE FACILITY LOCATION PROBLEM," The Engineering Economist, vol. 49, no. 3, pp. 237-252, Jan. 2004.

[45] M. C. Fu, "Feature Article: Optimization for simulation: Theory vs. Practice," INFORMS Journal on Computing, vol. 14, no. 3, pp. 192-215, Aug. 2002.

[46] S. J. Lim, S. J. Jeong, K. S. Kim, and M. W. Park, "A simulation approach for production-distribution planning with consideration given to replenishment policies," The International Journal of Advanced Manufacturing Technology, vol. 27, no. 5-6, pp. 593-603, Feb. 2005.

[47] M. Karatas and E. Yakıcı, "An analysis of p-median location problem: Effects of backup service level and demand assignment policy," European Journal of Operational Research, vol. 272, no. 1, pp. 207-218, 2019.

[48] D. Petrovic, R. Roy, and R. Petrovic, "Modelling and simulation of a supply chain in an uncertain environment," European Journal of Operational Research, vol. 109, no. 2, pp. 299-309, Sep. 1998.

[49] L.-Y. Ouyang, N.-C. Yeh, and K.-S. Wu, "Mixture inventory model with backorders and lost sales for variable lead time," Journal of the Operational Research Society, pp. 829-832, 1996.

[50] K. Aardal, Ö. Jonsson, and H. Jönsson, "Optimal Inventory Policies with Service-Level Constraints," Journal of the Operational Research Society, vol. 40, no. 1, pp. 65-73, Jan. 1989.

[51] M. van der Heijden, "Near cost-optimal inventory control policies for divergent networks under fill rate constraints," International Journal of Production Economics, vol. 63, no. 2, pp. 161-179, Jan. 2000. 
[52] D. Sculli and S. Y. Wu, "Stock Control with Two Suppliers and Normal Lead Times," The Journal of the Operational Research Society, vol. 32, no. 11, p. 1003, Nov. 1981.

[53] G. K. Janssens and K. M. Ramaekers, "A linear programming formulation for an inventory management decision problem with a service constraint," Expert Systems with Applications, vol. 38, no. 7, pp. 7929-7934, Jul. 2011.

[54] M. Goovaerts, "F. devylder, insurance premium," 1984.

[55] M. H. Cole, "Service considerations and the design of strategic distribution systems," Doctoral Dissertation, 1995.

[56] M. Shahabi, A. Tafreshian, A. Unnikrishnan, and S. D. Boyles, "Joint production-inventory-location problem with multi-variate normal demand," Transportation Research Part B: Methodological, vol. 110, pp. 60-78, 2018.

[57] F. J. Tapia-Ubeda, P. A. Miranda, and M. Macchi, "A generalized benders decomposition based algorithm for an inventory location problem with stochastic inventory capacity constraints," European Journal of Operational Research, vol. 267, no. 3, pp. 806-817, 2018.

[58] Z. Dai, F. Aqlan, X. Zheng, and K. Gao, "A location-inventory supply chain network model using two heuristic algorithms for perishable products with fuzzy constraints," Computers \& Industrial Engineering, vol. 119, pp. 338-352, 2018.

[59] M. S. Puga and J.-S. Tancrez, "A heuristic algorithm for solving large location-inventory problems with demand uncertainty," European Journal of Operational Research, vol. 259, no. 2, pp. 413-423, 2017.

[60] M. Amiri-Aref, W. Klibi, and M. Z. Babai, "The multi-sourcing location inventory problem with stochastic demand," European Journal of Operational Research, vol. 266, no. 1, pp. 72-87, 2018. 
[61] A. Diabat, E. Dehghani, and A. Jabbarzadeh, "Incorporating location and inventory decisions into a supply chain design problem with uncertain demands and lead times," Journal of Manufacturing Systems, vol. 43, pp. 139-149, 2017.

[62] X. Zheng, M. Yin, and Y. Zhang, "Integrated optimization of location, inventory and routing in supply chain network design," Transportation Research Part B: Methodological, vol. 121, pp. 1-20, 2019.

[63] A. Hiassat, A. Diabat, and I. Rahwan, "A genetic algorithm approach for location-inventory-routing problem with perishable products," Journal of manufacturing systems, vol. 42, pp. 93-103, 2017.

[64] Z. Drezner and H. W. Hamacher, Facility location: applications and theory. Springer Science \& Business Media, 2001.

[65] A. Harel, "Convexity Properties of the Erlang Loss Formula," Operations Research, vol. 38, no. 3, pp. 499-505, Jun. 1990.

[66] Q. Wang, R. Batta, and C. M. Rump, "Algorithms for a facility location problem with stochastic customer demand and immobile servers," Annals of operations Research, vol. 111, no. 1-4, pp. 17-34, 2002.

[67] M. L. Brandeau and S. S. Chiu, "Trajectory Analysis of the Stochastic Queue Median in a Plane with Rectilinear Distances," Transportation Science, vol. 24, no. 3, pp. 230-243, Aug. 1990.

[68] L.-R. Chen, "A Design of an Optimal Battery Pulse Charge System by Frequency-Varied Technique," IEEE Transactions on Industrial Electronics, vol. 54, no. 1, pp. 398-405, 2007.

[69] M. Bijvank and S. G. Johansen, "Periodic review lost-sales inventory models with compound Poisson demand and constant lead times of any length," European Journal of Operational Research, vol. 220, no. 1, pp. 106-114, Jul. 2012. 
[70] L. Liu and V. G. Kulkarni, "BALKING AND RENEGING IN M/G/s SYSTEMS EXACT ANALYSIS AND APPROXIMATIONS," Probability in the Engineering and Informational Sciences, vol. 22, no. 03, May 2008.

[71] R. Bekker and A. M. de Bruin, "Time-dependent analysis for refused admissions in clinical wards," Annals of Operations Research, vol. 178, no. 1, pp. 45-65, Jun. 2009.

[72] A. K. Erlang, "Solution of some problems in the theory of probabilities of significance in automatic telephone exchanges," Elektrotkeknikeren, vol. 13, pp. $5-13,1917$.

[73] D. Daley and C. Friedman, An Introduction to the Theory of Point Processes. New York: Springer-Verlag, 1998.

[74] J. L. Davis, W. A. Massey, and W. Whitt, "Sensitivity to the Service-Time Distribution in the Nonstationary Erlang Loss Model," Management Science, vol. 41, no. 6, pp. 1107-1116, Jun. 1995.

[75] A. M. de Bruin, R. Bekker, L. van Zanten, and G. M. Koole, "Dimensioning hospital wards using the Erlang loss model," Annals of Operations Research, vol. 178, no. 1, pp. 23-43, Oct. 2009.

[76] M. Restrepo, S. G. Henderson, and H. Topaloglu, "Erlang loss models for the static deployment of ambulances," Health Care Management Science, vol. 12, no. 1, pp. 67-79, Jul. 2008.

[77] N. M. van Dijk and N. Kortbeek, "Erlang loss bounds for OT-ICU systems," Queueing Systems, vol. 63, no. 1-4, pp. 253-280, Nov. 2009.

[78] S. Borst, A. Mandelbaum, and M. I. Reiman, "Dimensioning Large Call Centers," Operations Research, vol. 52, no. 1, pp. 17-34, Feb. 2004.

[79] W. Whitt, "Understanding the Efficiency of Multi-Server Service Systems," Management Science, vol. 38, no. 5, pp. 708-723, May 1992. 
[80] G. Zeng, "Two common properties of the erlang-B function, erlang-C function, and Engset blocking function," Mathematical and Computer Modelling, vol. 37, no. 12-13, pp. 1287-1296, Jun. 2003.

[81] F. Glover, "Future paths for integer programming and links to artificial intelligence," Computers $\&$ operations research, vol. 13, no. 5, pp. 533-549, 1986.

[82] P. R. Almeida, F. Soares, and J. A. P. Lopes, "Impacts of large-scale deployment of electric vehicles in the electric power system," in Electric Vehicle Integration into Modern Power Networks. Springer, 2013, pp. 203-249. 


\section{CURRICULUM VITAE}

NAME: Jie Zhang

ADDRESS: $\quad$ Department of Industrial Engineering

University of Louisville

Louisville, KY 40292

EDUCATION: B.S. Information Management

National Defense University (Shanghai)

2008

M.S. Industrial Engineering

State University of New York At Buffalo

2012

\section{PREVIOUS}

RESEARCH: Logistics

Operations Research

Optimization

AWARDS: $\quad$ American Society for Quality Scholarship Award - 2018

Alpha Pi Mu (Industrial Engineering Honor Society) 